\title{
Mechanistic Studies of the Allylic Rearrangements of $\alpha$-Silyloxy Allylic Silanes to $\alpha$-Silyloxy Vinylic Silanes
}

\author{
Angie I. Kim, Kyle L. Kimmel, Antonio Romero, Jacqueline H. Smitrovich, and K. A. Woerpel* \\ Department of Chemistry \\ University of California, Irvine \\ Irvine, CA 92697-2025
}

Supporting Information

\section{Contents:}

I. $\quad$ Synthesis of $( \pm) \alpha$-Silyloxy Allylic Silanes

S- 1

II. Synthesis of Acylsilane

S- 2

III. Synthesis of $( \pm) \alpha$-Pivalyloxy Allylic Silanes

S- 3

IV. Rearrangement of $\alpha$-Silyloxy Allylic Silanes

S- 3

V. Rearrangement of $\alpha$ - Pivalyloxy Allylic Silanes

S- 4

VI. Crossover Experiment of Rearrangement

VII. Stereochemistry Scrambling Experiment

VIII. Deprotection of $\alpha$-Silyloxy Vinylic Silanes

IX. References

X. Spectral Data

XI. Conformational Calculations

S- 5

S- 5

S- 6

S- 6

S- 6

S-65

General. Melting points were reported uncorrected. ${ }^{1} \mathrm{H}$ NMR and ${ }^{13} \mathrm{C}$ NMR spectra were recorded at ambient temperature at 500 and $400 \mathrm{MHz}$ and $125 \mathrm{MHz}$, respectively. The data are reported as follows: chemical shift in ppm from internal tetramethylsilane on the $\delta$ scale, multiplicity $(\mathrm{br}=$ broad, $\mathrm{s}=$ singlet, $\mathrm{d}=$ doublet, $\mathrm{t}=$ triplet, $\mathrm{q}=$ quartet, $\mathrm{m}=$ multiplet), coupling constants $(\mathrm{Hz})$, and integration. High resolution mass spectra were obtained by peak matching. Liquid chromatography was performed using forced flow (flash chromatography) of the indicated solvent system on silica gel $\left(\mathrm{SiO}_{2}\right) 60$ (230-400 mesh). Enantiomeric excess were determined by HPLC analysis, and the enantiomerically enriched material was compared with racemic material. All reactions were carried out under an atmosphere of nitrogen in glassware that had been flame-dried under vacuum $(0.05 \mathrm{~mm} \mathrm{Hg})$. Unless otherwise noted, all reagents were commercially obtained and, where appropriate, purified prior to use. THF, $\mathrm{Et} 2 \mathrm{O}$, toluene, and $\mathrm{CH}_{2} \mathrm{Cl}_{2}$ were dried by filtration through alumina. ${ }^{1}$

\section{Synthesis of $\alpha$-Silyloxy Allylic Silanes}
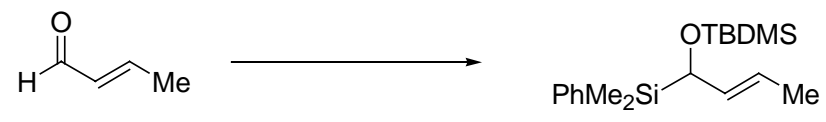

1

Representative Procedure for the Isolation of $\alpha$-Siloxy Allylic Silanes:

a-Silyloxy Allylic Silane 1. To a solution of $\mathrm{PhMe}_{2} \mathrm{SiCl}(7.00 \mathrm{~g}, 41.0 \mathrm{mmol}, 1.15$ equiv.) in $120 \mathrm{~mL}$ of THF $(0.35 \mathrm{M})$ was added finely cut lithium wire $\left(2.27 \mathrm{~g}, 328 \mathrm{mmol}, 9.2\right.$ equiv.). The suspension was stirred at $24{ }^{\circ} \mathrm{C}$ for $18 \mathrm{~h}$. The resultant $\mathrm{PhMe}_{2} \mathrm{SiLi}^{2}$ solution was transferred by cannula to a clean flask and cooled to $-78^{\circ} \mathrm{C}$. Crotonaldehyde $(2.52 \mathrm{~g}, 35.7 \mathrm{mmol}, 1.00 \mathrm{equiv}$.) in $20 \mathrm{~mL}$ of THF (1.2 M) was then added dropwise. The solution was stirred for $20 \mathrm{~min}$ at $-78{ }^{\circ} \mathrm{C}$, and then the reaction mixture was added to saturated aqueous $\mathrm{NH}_{4} \mathrm{Cl}(350 \mathrm{~mL})$. The resultant layers were separated and the aqueous layer was extracted with $\mathrm{Et}_{2} \mathrm{O}(3 \times 150 \mathrm{~mL})$. The combined organic layers were washed with saturated aqueous $\mathrm{NaCl}(300 \mathrm{~mL})$. The resultant organic phase was dried over $\mathrm{MgSO}_{4}$ and filtered. The filtrate was concentrated in vacuo to afford the $\alpha$-hydroxy allylic silane as a yellow oil. The oil was then dissolved in $16 \mathrm{ml}$ of anhydrous DMF (2 M) followed by the addition of TBDMSCl (5.66 g, $37.6 \mathrm{mmol}, 1.05$ equiv.) and imidazole ( $4.99 \mathrm{~g}, 73.4 \mathrm{mmol}, 2.05$ equiv.). The solution was allowed to stir at $23{ }^{\circ} \mathrm{C}$ for a minimum of $12 \mathrm{~h}$. The reaction mixture was diluted with hexanes $(400 \mathrm{ml})$ and the resultant solution was washed with saturated aqueous $\mathrm{NH}_{4} \mathrm{Cl}(350 \mathrm{~mL})$. The layers were separated and the aqueous layer was extracted with hexanes $(3 \times 200 \mathrm{~mL})$. The combined organic layers were washed with saturated aqueous $\mathrm{NaCl}$ $(400 \mathrm{~mL})$. The resultant organic phase was dried over $\mathrm{MgSO}_{4}$ and filtered. The filtrate was concentrated in vacuo to afford the $\alpha-$ silyloxy allylic silane as a light yellow oil. Purification by flash chromatography (hexanes) afforded $\mathbf{1}$ as a colorless oil (5.86 g, 52\%): $\mathrm{GC} \mathrm{t}_{\mathrm{R}} 12.4 \mathrm{~min}\left(50{ }^{\circ} \mathrm{C}, 10^{\circ} \mathrm{C} / \mathrm{min}\right) ;{ }^{1} \mathrm{H}$ NMR $\left(500 \mathrm{MHz}, \mathrm{CDCl}_{3}\right) \delta 7.55(\mathrm{~m}, 2 \mathrm{H}), 7.35(\mathrm{~m}, 3 \mathrm{H}), 5.42(\mathrm{~m}, 2 \mathrm{H}), 4.04(\mathrm{dt}, J=6.1,1.5$ $\mathrm{Hz}, 1 \mathrm{H}), 1.66(\mathrm{dt}, J=6.1,1.3 \mathrm{~Hz}, 3 \mathrm{H}), 0.88(\mathrm{~s}, 9 \mathrm{H}), 0.30(\mathrm{~s}, 3 \mathrm{H}), 0.28(\mathrm{~s}, 3 \mathrm{H}),-0.04(\mathrm{~s}, 3 \mathrm{H}),-0.11(\mathrm{~s}, 3 \mathrm{H}) ;{ }^{13} \mathrm{C} \mathrm{NMR}(125 \mathrm{MHz}$, $\left.\mathrm{CDCl}_{3}\right) \delta 137.7,134.6,132.2,129.2,127.7,122.2,68.4,26.1,18.4,18.0,-4.1,-5.0,-5.3,-5.6$; IR (thin film) $2957,1472,1252,1084$ 
$\mathrm{cm}^{-1}$; HRMS (ESI) $\mathrm{m} / \mathrm{z}$ calcd for $\mathrm{C}_{18} \mathrm{H}_{32} \mathrm{OSi}_{2} \mathrm{Na}(\mathrm{M}+\mathrm{Na})^{+}$343.1889, found 343.1888. Anal. Calcd for $\mathrm{C}_{18} \mathrm{H}_{32} \mathrm{OSi}_{2}: \mathrm{C}, 67.43 ; \mathrm{H}$, 10.06. Found: C, 67.23; H, 10.21.

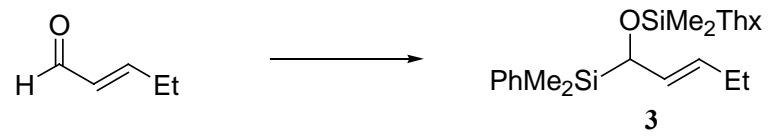

$\alpha$-Silyloxy Allylic Silane 3. The representative procedure was followed using $\mathrm{PhMe}_{2} \mathrm{SiCl}$ (4.96 g, $\left.29.0 \mathrm{mmol}\right)$, lithium wire (3.22 g, $464 \mathrm{mmol})$, and trans-2-pentenal $(2.03 \mathrm{~g}, 24.1 \mathrm{mmol})$. Protection was performed using thexyldimethylsilyl chloride $(5.18 \mathrm{~g}, 29.0$ $\mathrm{mmol})$ and imidazole $(3.87 \mathrm{~g}, 56.5 \mathrm{mmol})$. Purification by flash chromatography (hexanes) afforded 3 as a colorless oil $(5.00 \mathrm{~g}, 57 \%)$ : $\mathrm{GC} \mathrm{t}_{\mathrm{R}} 15.5 \mathrm{~min}\left(50{ }^{\circ} \mathrm{C}, 10^{\circ} \mathrm{C} / \mathrm{min}\right) ;{ }^{1} \mathrm{H}$ NMR $\left(500 \mathrm{MHz}, \mathrm{CDCl}_{3}\right) \delta 7.55(\mathrm{~m}, 2 \mathrm{H}), 7.35(\mathrm{~m}, 3 \mathrm{H}), 5.42(\mathrm{~m}, 2 \mathrm{H}) 4.05(\mathrm{~m}, 1 \mathrm{H}), 2.0(\mathrm{~m}$, 2H), 1.68 (septet, $J=6.9 \mathrm{~Hz}, 1 \mathrm{H}), 0.95(\mathrm{t}, J=7.4 \mathrm{~Hz}, 3 \mathrm{H}), 0.90(\mathrm{~d}, J=6.8 \mathrm{~Hz}, 3 \mathrm{H}), 0.88(\mathrm{~d}, J=6.8 \mathrm{~Hz}, 3 \mathrm{H}), 0.83(\mathrm{~s}, 3 \mathrm{H}), 0.82(\mathrm{~s}$, $3 \mathrm{H}), 0.38(\mathrm{~s}, 3 \mathrm{H}), 0.32(\mathrm{~s}, 3 \mathrm{H}), 0.01(\mathrm{~s}, 3 \mathrm{H}),-0.05(\mathrm{~s}, 3 \mathrm{H}) ;{ }^{13} \mathrm{C}$ NMR $\left(125 \mathrm{MHz}, \mathrm{CDCl}_{3}\right) \delta$ 137.7, 134.6, 130.3, 129.6, 129.2, 127.6, 68.5, 34.4, 25.7, 25.3, 20.7, 20.6, 18.9, 18.8, 14.4, -1.9, -3.1, -5.3, -5.6; IR (thin film) 2959, 1464, 1251, $1028 \mathrm{~cm}^{-1}$; Anal. Calcd for $\mathrm{C}_{21} \mathrm{H}_{38} \mathrm{OSi}_{2}: \mathrm{C}, 69.54 ; \mathrm{H}, 10.56$. Found: $\mathrm{C}, 69.65 ; \mathrm{H}, 10.75$.

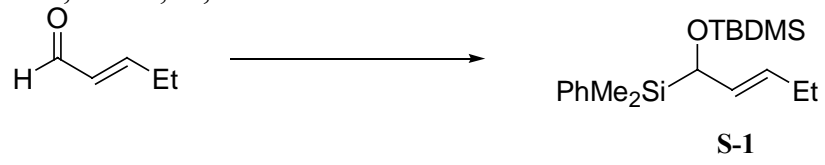

$\alpha$-Silyloxy Allylic Silane S-1. The representative procedure was followed using $\mathrm{PhMe}_{2} \mathrm{SiCl}$ (2.09 g, $\left.12.2 \mathrm{mmol}\right)$, lithium wire (1.36 $\mathrm{g}, 196 \mathrm{mmol})$, and trans-2-pentenal $(0.86 \mathrm{~g}, 10.2 \mathrm{mmol})$. Protection was performed using TBDMSCl $(1.84 \mathrm{~g}, 12.2 \mathrm{mmol})$ and imidazole $(1.64 \mathrm{~g}, 23.9 \mathrm{mmol})$. Purification by flash chromatography (hexanes) afforded $\mathbf{S}-\mathbf{1}$ as a colorless oil $(1.14 \mathrm{~g}, 34 \%)$ : GC $\mathrm{t}_{\mathrm{R}}$ $13.3 \mathrm{~min}\left(50{ }^{\circ} \mathrm{C}, 10{ }^{\circ} \mathrm{C} / \mathrm{min}\right) ;{ }^{1} \mathrm{H}$ NMR $\left(500 \mathrm{MHz}, \mathrm{CDCl}_{3}\right) \delta 7.31-7.54(\mathrm{~m}, 5 \mathrm{H}), 5.42(\mathrm{~m}, 2 \mathrm{H}), 4.04(\mathrm{~m}, 1 \mathrm{H}), 1.98(\mathrm{~m}, 2 \mathrm{H}), 0.93(\mathrm{t}, J$ $=7.5 \mathrm{~Hz}, 3 \mathrm{H}), 0.86(\mathrm{~s}, 9 \mathrm{H}), 0.29(\mathrm{~s}, 3 \mathrm{H}), 0.26(\mathrm{~s}, 3 \mathrm{H}),-0.06(\mathrm{~s}, 3 \mathrm{H}),-0.13(\mathrm{~s}, 3 \mathrm{H}) ;{ }^{13} \mathrm{C} \mathrm{NMR}\left(125 \mathrm{MHz}, \mathrm{CDCl}_{3}\right) \delta 137.4,134.3$, $129.9,129.2,129.0,127.4,68.2,25.9,25.5,18.2,14.2,-4.2,-5.3,-5.6,-5.9$; IR (thin film) $2958,1253,836 \mathrm{~cm}^{-1}$; HRMS (APCI) $\mathrm{m} /$ $z$ calcd for $\mathrm{C}_{19} \mathrm{H}_{34} \mathrm{OSi}_{2}(\mathrm{M}-\mathrm{H})^{+}$333.2070, found 333.2064. Anal. Calcd for $\mathrm{C}_{19} \mathrm{H}_{33} \mathrm{OSi}_{2}: \mathrm{C}, 68.19 ; \mathrm{H}, 10.24$. Found: $\mathrm{C}, 68.19 ; \mathrm{H}$, 10.15 .

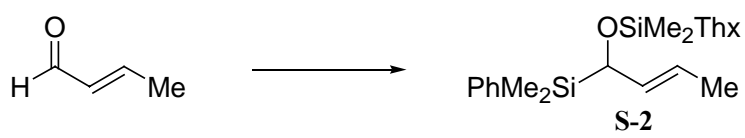

$\alpha$-Silyloxy Allylic Silane S-2. The representative procedure was followed using $\mathrm{PhMe}_{2} \mathrm{SiCl}$ (1.12 g, $\left.6.55 \mathrm{mmol}\right)$, lithium wire (0.068 g, $9.73 \mathrm{mmol})$, and crotonaldehyde $(0.500 \mathrm{~g}, 6.03 \mathrm{mmol})$. Protection was performed using thexyldimethylsilyl chloride $(1.20 \mathrm{~mL}$, $6.10 \mathrm{mmol})$ and imidazole $(0.82 \mathrm{~g}, 12.0 \mathrm{mmol})$. Purification by flash chromatography (hexanes) afforded $\mathbf{S}-\mathbf{2}$ as a colorless oil (1.43 g, 69\%): GC t $14.7 \mathrm{~min}\left(50{ }^{\circ} \mathrm{C}, 10^{\circ} \mathrm{C} / \mathrm{min}\right) ;{ }^{1} \mathrm{H}$ NMR $\left(500 \mathrm{MHz}, \mathrm{CDCl}_{3}\right) \delta 7.33-7.54(\mathrm{~m}, 5 \mathrm{H}), 5.41(\mathrm{~m}, 2 \mathrm{H}), 4.03(\mathrm{~m}, 1 \mathrm{H}), 1.64(\mathrm{~d}$, $\mathrm{J}=6.0 \mathrm{~Hz}, 3 \mathrm{H}), 1.60($ septet, $J=6.8 \mathrm{~Hz}, 1 \mathrm{H}), 0.87(\mathrm{~d}, J=6.8 \mathrm{~Hz}, 3 \mathrm{H}), 0.85(\mathrm{~d}, J=6.8 \mathrm{~Hz}, 3 \mathrm{H}), 0.80(\mathrm{~s}, 3 \mathrm{H}), 079(\mathrm{~s}, 3 \mathrm{H}), 0.29(\mathrm{~s}$, $3 \mathrm{H}), 0.25(\mathrm{~s}, 3 \mathrm{H}),-0.02(\mathrm{~s}, 3 \mathrm{H}),-0.11(\mathrm{~s}, 3 \mathrm{H}) ;{ }^{13} \mathrm{C} \mathrm{NMR}\left(125 \mathrm{MHz}, \mathrm{CDCl}_{3}\right) \delta 137.8,134.7,132.4,129.3,127.7,122.3,68.6,34.5$, 25.2, 20.8, 20.7, 19.0, 18.8, 18.1, -2.0,-3.0, -5.1, -5.5; IR (thin film) 2958, 1465, 1249, $1081 \mathrm{~cm}^{-1}$; HRMS (CI/isobutane) $\mathrm{m} / \mathrm{z}$ calcd for $\mathrm{C}_{20} \mathrm{H}_{37} \mathrm{OSi}_{2}(\mathrm{M}+\mathrm{H})^{+}$348.2305, found 348.2299. Anal. Calcd for $\mathrm{C}_{20} \mathrm{H}_{36} \mathrm{OSi}_{2}$ : C, 68.89; H, 10.41. Found: C, 69.13; $\mathrm{H}, 10.45$.

\section{Synthesis of Acylsilane}

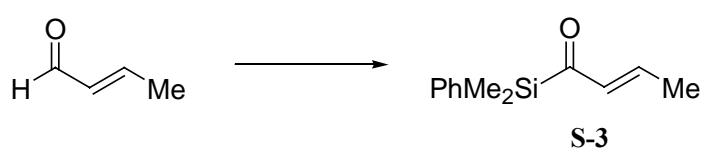

Acylsilane S-3. To a solution of $\mathrm{PhMe}_{2} \mathrm{SiCl}(10.0 \mathrm{~g}, 58.5 \mathrm{mmol}, 1.2$ equiv) in anhydrous THF (165 mL, $0.35 \mathrm{M})$ was added lithium wire $\left(7.01 \mathrm{~g}, 1.01 \mathrm{~mol}, 21\right.$ equiv.). The suspension was stirred at $24^{\circ} \mathrm{C}$ for $18 \mathrm{~h}$. The resultant $\mathrm{PhMe}_{2} \mathrm{SiLi}$ solution was transferred by cannula to a clean flask and cooled to $-78{ }^{\circ} \mathrm{C}$. Crotonaldehyde $(3.40 \mathrm{~g}, 48.5 \mathrm{mmol}, 1.0$ equiv.) was then added dropwise. The solution was stirred for $20 \mathrm{~min}$ at $-78^{\circ} \mathrm{C}$ then the reaction mixture was added into saturated aqueous $\mathrm{NH}_{4} \mathrm{Cl}(500 \mathrm{~mL})$. The resultant layers were separated and the aqueous layer was extracted with $\mathrm{Et}_{2} \mathrm{O}(3 \times 250 \mathrm{~mL})$. The combined organic layers were washed with saturated aqueous $\mathrm{NaCl}(400 \mathrm{~mL})$. The resultant organic phase was dried over $\mathrm{MgSO}_{4}$ and filtered. The filtrate was concentrated in vacuo to afford the $\alpha$-hydroxy allylic silane as a yellow oil. In a separate flask, containing a solution of oxalyl chloride $(7.11 \mathrm{~g}, 56.0$ mmol, 1.15 equiv.) in $130 \mathrm{~mL}$ of $\mathrm{CH}_{2} \mathrm{Cl}_{2}$ at $-78{ }^{\circ} \mathrm{C}$, was added DMSO (9.57 g, $122 \mathrm{mmol}, 2.5$ equiv.) in $50 \mathrm{~mL}$ of $\mathrm{CH}_{2} \mathrm{Cl}_{2}$ dropwise over 30 min. After stirring for 30 minutes at $-78^{\circ} \mathrm{C}$, the $\alpha$-hydroxy allylic silane in $70 \mathrm{~mL} \mathrm{of} \mathrm{CH}_{2} \mathrm{Cl}_{2}$ was added over 45 min. After stirring for $1 \mathrm{~h}$ at $-78{ }^{\circ} \mathrm{C}$, triethylamine $(26.1 \mathrm{~g}, 255 \mathrm{mmol}, 5.20$ equiv. $)$ was added over $10 \mathrm{~min}$. The solution was stirred at $-78{ }^{\circ} \mathrm{C}$ for $1 \mathrm{~h}$ then poured into a 1:3 mixture of $\mathrm{H}_{2} \mathrm{O}$ :pentane $(160 \mathrm{ml})$. The layers were separated and the aqueous layer was extracted with $\mathrm{Et}_{2} \mathrm{O}(2 \times 100 \mathrm{~mL})$. The combined organic layers were washed with $4 \times 60 \mathrm{~mL}$ of $1 \mathrm{~N}$ aqueous $\mathrm{HCl}, 4 \times 60 \mathrm{~mL}$ of $\mathrm{H}_{2} \mathrm{O}$, and $2 \times 60$ $\mathrm{mL}$ of saturated aqueous $\mathrm{NaCl}$. The resultant organic phase was dried over $\mathrm{MgSO}_{4}$ and filtered. The filtrate was concentrated in vacuo to afford the acylsilane as a bright yellow oil. Purification by flash chromatography (95:5 hexanes:EtOAc) afforded S-3 as a vibrant yellow oil (5.07 g, 56\%): GC t $\mathrm{R}_{\mathrm{R}} 9.2 \mathrm{~min}\left(50{ }^{\circ} \mathrm{C}, 10^{\circ} \mathrm{C} / \mathrm{min}\right) ;{ }^{1} \mathrm{H} \mathrm{NMR}\left(500 \mathrm{MHz}, \mathrm{CDCl}_{3}\right) \delta 7.53(\mathrm{~m}, 2 \mathrm{H}), 7.38(\mathrm{~m}, 3 \mathrm{H}), 6.66$ 
$\left.(\mathrm{dq}, J=16.0,6.8 \mathrm{~Hz}, 1 \mathrm{H}), 6.25(\mathrm{dq}, J=16.0,1.5 \mathrm{~Hz}, 1 \mathrm{H}), 1.82(\mathrm{dd}, J=6.8,1.6 \mathrm{~Hz}, 3 \mathrm{H}), 0.52(\mathrm{~s}, 6 \mathrm{H}) ;{ }^{13} \mathrm{C} \mathrm{NMR}(125 \mathrm{MHz}, \mathrm{CDCl})\right) \delta$ $234.3,144.9,138.0,135.6,134.3,129.9,128.2,18.7,18.0,-3.5$; IR (thin film) $2966,1648,1596 \mathrm{~cm}^{-1}$; HRMS (ESI) m / z calcd for $\mathrm{C}_{12} \mathrm{H}_{16} \mathrm{OSiNa}(\mathrm{M}+\mathrm{Na})^{+} 227.0868$, found 227.0869. Anal. Calcd for $\mathrm{C}_{12} \mathrm{H}_{16} \mathrm{OSi}$ : C, 70.53; H, 7.89. Found: C, 70.24; H, 7.86.

\section{Synthesis of $( \pm) \alpha$-Pivalyloxy Allylic Silanes and $( \pm) \alpha$-Pivalyloxy Vinylic Silanes}

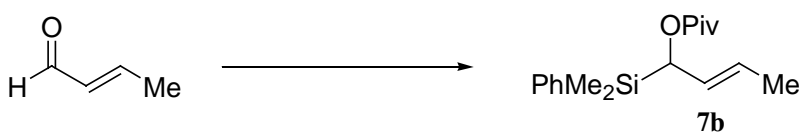

a-Pivalyloxy Allylic Silane 7b. To a solution of $\left.\mathrm{PhMe} \mathrm{SiCl}_{2} \mathrm{Si.04} \mathrm{g}, 6.11 \mathrm{mmol}\right)$ in $15 \mathrm{~mL}$ of THF was added lithium wire (0.282 g, $40.7 \mathrm{mmol}$ ). The suspension was stirred at room temperature for $18 \mathrm{~h}$. The resultant $\mathrm{PhMe}_{2} \mathrm{SiLi}$ solution was transferred by cannula to a clean flask and cooled to $-78^{\circ} \mathrm{C}$. Crotonaldehyde $(0.356 \mathrm{~g}, 5.09 \mathrm{mmol})$ in $1 \mathrm{~mL}$ of THF was then added dropwise. The solution was stirred for $45 \mathrm{~min}$ at $-78{ }^{\circ} \mathrm{C}$ then the reaction mixture was added into saturated aqueous $\mathrm{NH}_{4} \mathrm{Cl}(45 \mathrm{~mL})$. The layers were separated and the aqueous layer was extracted with $3 \times 40 \mathrm{~mL}$ of $\mathrm{Et}_{2} \mathrm{O}$. The combined organic layers were washed with saturated aqueous $\mathrm{NaCl}(80 \mathrm{~mL})$. The resultant organic phase was dried over $\mathrm{MgSO}_{4}$ and filtered. The filtrate was concentrated in vacuo to afford the $\alpha$-hydroxy allylic alcohol as a yellow oil. In a separate flask containing pivalic anhydride (1.90 g, $10.2 \mathrm{mmol}) \mathrm{in} 20 \mathrm{~mL}$ of $\mathrm{CH}_{2} \mathrm{Cl}_{2}$ was added anhydrous $\mathrm{MgBr}_{2}(1.87 \mathrm{~g}, 10.2 \mathrm{mmol})$ in $20 \mathrm{~mL}$ of $\mathrm{CH}_{2} \mathrm{Cl}_{2}$. The solution was allowed to stir for $10 \mathrm{minutes}$ at 23 ${ }^{\circ} \mathrm{C}$. The reaction mixture was then treated with triethylamine $(2.12 \mathrm{~mL}, 15.3 \mathrm{mmol})$ followed by the crude $\alpha$-hydroxy allylic silane. After $14 \mathrm{~h}$ at $23{ }^{\circ} \mathrm{C}, 40 \mathrm{~mL}$ of $\mathrm{H}_{2} \mathrm{O}$ was added. The layers were separated and the aqueous layer was extracted with $3 \times 80 \mathrm{~mL}$ of EtOAc. The combined organic layers were washed with saturated aqueous $\mathrm{NaCl}(150 \mathrm{~mL})$. The resultant organic phase was dried over $\mathrm{MgSO}_{4}$ and filtered. The filtrate was concentrated in vacuo to afford unpurified $\mathbf{7 b}$ as a light yellow oil. Purification by flash chromatography (4:96 EtOAc:hexanes) afforded $7 \mathbf{b}$ as a colorless oil $(1.29 \mathrm{~g}, 87 \%)$ : GC t $12.0 \mathrm{~min}\left(50{ }^{\circ} \mathrm{C}, 10{ }^{\circ} \mathrm{C} / \mathrm{min}\right)$; ${ }^{1} \mathrm{H} \mathrm{NMR}$ $\left(500 \mathrm{MHz} \mathrm{CDCl}_{3}\right) \delta 7.52(\mathrm{~m}, 2 \mathrm{H}), 7.36(\mathrm{~m}, 3 \mathrm{H}), 5.47(\mathrm{~m}, 2 \mathrm{H}), 5.33(\mathrm{~m}, 1 \mathrm{H}), 1.70(\mathrm{~s}, 3 \mathrm{H}), 1.22(\mathrm{~s}, 9 \mathrm{H}), 0.39(\mathrm{~s}, 3 \mathrm{H}), 0.38(\mathrm{~s}, 3 \mathrm{H}) ;{ }^{13} \mathrm{C}$ NMR $\left(125 \mathrm{MHz}_{\mathrm{CDCl}}\right) \delta 178.0,135.8,134.2,129.5,127.8,127.4,124.4,68.9,39.0,27.3,18.0,-5.1$, -5.5; IR (thin film) 2962, $1727,1150 \mathrm{~cm}^{-1}$; HRMS (ESI) $\mathrm{m} / \mathrm{z}$ calcd for $\mathrm{C}_{17} \mathrm{H}_{26} \mathrm{O}_{2} \mathrm{SiNa}(\mathrm{M}+\mathrm{Na})^{+}$313.1600, found 313.1594. Anal. Calcd for $\mathrm{C}_{17} \mathrm{H}_{26} \mathrm{O}_{2} \mathrm{Si}$ : $\mathrm{C}$, 70.29; H, 9.02. Found: C, 70.25; H, 9.14.

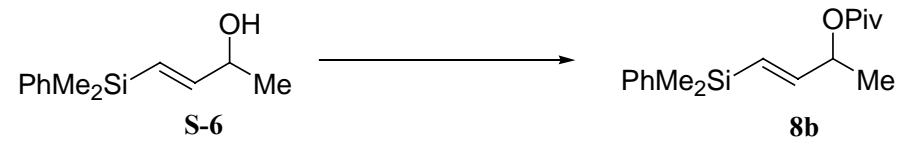

a-Pivalyloxy Vinylic Silane 8b. To a $\left(23{ }^{\circ} \mathrm{C}\right)$ solution of pivalic anhydride $\left(0.471 \mathrm{~g}, 2.53 \mathrm{mmol}^{2}\right.$ in $5 \mathrm{~mL}$ of $\mathrm{CH}_{2} \mathrm{Cl}_{2}$ was added anhydrous $\mathrm{MgBr}_{2}(0.466 \mathrm{~g}, 2.53 \mathrm{mmol})$ in $5 \mathrm{~mL}$ of $\mathrm{CH}_{2} \mathrm{Cl}_{2}$. The solution was allowed to stir for $10 \mathrm{minutes}$ at $23{ }^{\circ} \mathrm{C}$. The reaction mixture was then treated with triethylamine $(0.530 \mathrm{~mL}, 3.79 \mathrm{mmol})$ followed by allylic alcohol $\mathbf{S}-6$. After $19 \mathrm{hrs}$ at $23{ }^{\circ} \mathrm{C}, 15 \mathrm{~mL}$ of $\mathrm{H}_{2} \mathrm{O}$ was added. The layers were separated and the aqueous layer was re-extracted with $(3 \times 30 \mathrm{~mL})$ of EtOAc. The combined organic layers were washed with saturated aqueous $\mathrm{NaCl}(150 \mathrm{~mL})$. The resultant organic phase was dried over $\mathrm{MgSO}_{4}$ and filtered. The filtrate was concentrated in vacuo to afford unpurified $\mathbf{8 b}$ as a light yellow oil. Purification by flash chromatography (8:92 EtOAc:hexanes) afforded $\mathbf{8 b}$ as a colorless oil $(0.337 \mathrm{~g}, 91 \%)$ : $\mathrm{GC} \mathrm{t}_{\mathrm{R}} 12.3 \mathrm{~min}\left(50{ }^{\circ} \mathrm{C}, 10^{\circ} \mathrm{C} / \mathrm{min}\right) ;{ }^{1} \mathrm{H} \mathrm{NMR}\left(500 \mathrm{MHz}, \mathrm{CDCl}{ }_{3}\right) \delta$ $7.52(\mathrm{~m}, 2 \mathrm{H}), 7.37(\mathrm{~m}, 3 \mathrm{H}), 6.10(\mathrm{dd}, J=18.8,4.6 \mathrm{~Hz}, 1 \mathrm{H}), 6.0(\mathrm{dd}, J=18.8,1.3 \mathrm{~Hz}, 1 \mathrm{H}), 5.38(\mathrm{~m}, 1 \mathrm{H}), 1.30(\mathrm{~d}, \mathrm{~J}=6.6 \mathrm{~Hz}, 3 \mathrm{H}), 1.22$ $(\mathrm{s}, 9 \mathrm{H}), 0.36(\mathrm{~s}, 6 \mathrm{H}) ;{ }^{13} \mathrm{C} \mathrm{NMR}\left(125 \mathrm{MHz}, \mathrm{CDCl}_{3}\right) \delta 178.0,147.3,138.7,134.1,129.4,128.2,127.9,71.9,39.1,27.5,20.0,-2.2$, -2.3; IR (thin film) 2976, 1729, $1159 \mathrm{~cm}^{-1}$; HRMS (ESI) $\mathrm{m} / \mathrm{z}$ calcd for $\mathrm{C}_{17} \mathrm{H}_{26} \mathrm{O}_{2} \mathrm{SiNa}(\mathrm{M}+\mathrm{Na})^{+} 313.1600$, found 313.1591. Anal. Calcd for $\mathrm{C}_{17} \mathrm{H}_{26} \mathrm{O}_{2} \mathrm{Si}$ : C, 70.29; H, 9.02. Found: C, 70.29; H, 9.03.

\section{Rearrangement of $( \pm) \alpha$-Silyloxy Allylic Silanes}

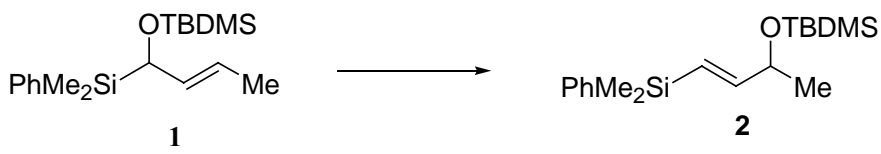

\section{Representative Procedure for $\alpha$-Silyloxy Allylic Silane Rearrangement:}

$\alpha$-Silyloxy Vinylic Silane 2. To a cooled $\left(-78^{\circ} \mathrm{C}\right)$ solution of $\alpha$-silyloxy allylic silane $(7.2 \mathrm{~g}, 22.5 \mathrm{mmol}, 1.0 \mathrm{equiv}$.) in $80 \mathrm{~mL}$ of $\mathrm{CH}_{2} \mathrm{Cl}_{2}(0.14 \mathrm{M})$ was added $\mathrm{BF}_{3} \cdot \mathrm{OEt}_{2}\left(3.51 \mathrm{~g}, 24.7 \mathrm{mmol}, 1.1\right.$ equiv.). After 3 hours at $-78^{\circ} \mathrm{C}$, an aqueous solution of $\mathrm{NaHCO}_{3}(300$ $\mathrm{mL})$ was added. The resultant layers were separated and the aqueous layer was extracted with $(3 \times 100 \mathrm{~mL})$ of hexanes. The combined organic layers were washed with saturated aqueous $\mathrm{NaCl}(250 \mathrm{~mL})$, dried over $\mathrm{MgSO}_{4}$, and filtered. The filtrate was concentrated in vacuo to afford the $\alpha$-silyloxy vinylic silane as a light yellow oil. Vinylic silane 2 was purified by silica gel chromatography (EtOAc/hexanes). Purification by flash chromatography (2:98 EtOAc:hexanes) afforded 2 as a colorless oil (6.04 g, 84\%): $\mathrm{GC} \mathrm{t}_{\mathrm{R}} 12.8 \mathrm{~min}\left(50{ }^{\circ} \mathrm{C}, 10{ }^{\circ} \mathrm{C} / \mathrm{min}\right) ;{ }^{1} \mathrm{H} \mathrm{NMR}\left(500 \mathrm{MHz}, \mathrm{CDCl}_{3}\right) \delta 7.54(\mathrm{~m}, 2 \mathrm{H}), 7.37(\mathrm{~m}, 3 \mathrm{H}), 6.15(\mathrm{dd}, J=18.6,4.4 \mathrm{~Hz}$, 
$1 \mathrm{H}), 5.95(\mathrm{~d}, J=18.6 \mathrm{~Hz}, 1 \mathrm{H}), 4.35(\mathrm{~m}, 1 \mathrm{H}), 1.23(\mathrm{~d}, J=6.4 \mathrm{~Hz}, 3 \mathrm{H}), 0.92(\mathrm{~s}, 9 \mathrm{H}), 0.35(\mathrm{~s}, 6 \mathrm{H}), 0.07(\mathrm{~s}, 6 \mathrm{H}) ;{ }^{13} \mathrm{C} \mathrm{NMR}(125 \mathrm{MHz}$, $\left.\mathrm{CDCl}_{3}\right) \delta 152.5,139.3,134.2,129.3,128.1,124.9,71.5,26.3,24.3,18.7,-2.2,-2.3,-4.3,-4.4 ;$ IR (thin film) $2956,2857,1620,1250$ $\mathrm{cm}^{-1}$; HRMS (ESI) $\mathrm{m} / \mathrm{z}$ calcd for $\mathrm{C}_{18} \mathrm{H}_{32} \mathrm{OSi}_{2} \mathrm{Na}(\mathrm{M}+\mathrm{Na})^{+} 343.1889$, found 343.1886 .

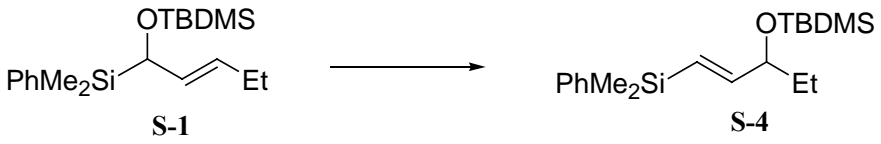

$\boldsymbol{\alpha}$-Silyloxy Vinylic Silane S-4. The representative procedure for the $\alpha$-silyloxy allylic silane rearrangement was followed using $\alpha$ silyloxy allylic silane $(0.400 \mathrm{~g}, 1.20 \mathrm{mmol}), \mathrm{CH}_{2} \mathrm{Cl}_{2}(5 \mathrm{~mL})$, and $\mathrm{BF}_{3} \cdot \mathrm{OEt}_{2}(0.177 \mathrm{~g}, 1.24 \mathrm{mmol})$. Purification by flash chromatography (2:98 EtOAc:hexanes) afforded $\mathbf{S}-4$ as a colorless oil $(0.283 \mathrm{~g}, 71 \%)$ : GC $\mathrm{t}_{\mathrm{R}} 13.7 \mathrm{~min}\left(50{ }^{\circ} \mathrm{C}, 10{ }^{\circ} \mathrm{C} / \mathrm{min}\right) ;{ }^{1} \mathrm{H} \mathrm{NMR}$ $\left(500 \mathrm{MHz}, \mathrm{CDCl}_{3}\right) \delta 7.54(\mathrm{~m}, 2 \mathrm{H}), 7.37(\mathrm{~m}, 3 \mathrm{H}), 6.07(\mathrm{dd}, J=18.7,5.4 \mathrm{~Hz}, 1 \mathrm{H}), 5.91(\mathrm{~d}, J=18.7 \mathrm{~Hz}, 1 \mathrm{H}), 4.05(\mathrm{~m}, 1 \mathrm{H}), 1.51(\mathrm{~m}$, $2 \mathrm{H}), 0.90(\mathrm{~m}, 9 \mathrm{H}), 0.89(\mathrm{~m}, 3 \mathrm{H}), 0.34(\mathrm{~s}, 6 \mathrm{H}), 0.05(\mathrm{~s}, 3 \mathrm{H}), 0.03(\mathrm{~s}, 3 \mathrm{H}) ;{ }^{13} \mathrm{C} \mathrm{NMR}\left(125 \mathrm{MHz}, \mathrm{CDCl}_{3}\right) \delta 151.4,139.2,134.0,129.0$, $127.9,126.2,77.0,30.8,26.1,18.6,9.9,-2.2,-2.3,-4.1,-4.5$; IR (thin film) 2958, 2857, $1250,836 \mathrm{~cm}^{-1}$; HRMS (ESI) $\mathrm{m} / \mathrm{z}$ calcd for $\mathrm{C}_{19} \mathrm{H}_{34} \mathrm{OSi}_{2} \mathrm{Na}(\mathrm{M}+\mathrm{Na})^{+}$357.2046, found 357.2039. Anal. Calcd for $\mathrm{C}_{19} \mathrm{H}_{34} \mathrm{OSi}_{2}$ : C, 68.19; H, 10.24. Found: C, 68.29; H, 10.18.

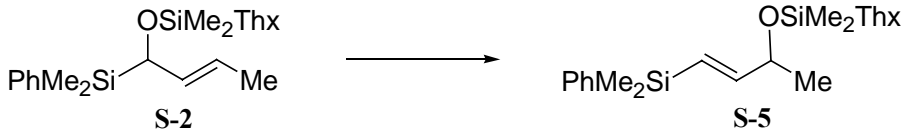

$\boldsymbol{\alpha}$-Silyloxy Vinylic Silane S-5. As above, the representative procedure for $\alpha$-silyloxy allylic silane rearrangement was followed using $\alpha$-silyloxy allylic silane $(0.500 \mathrm{~g}, 1.43 \mathrm{mmol}), \mathrm{CH}_{2} \mathrm{Cl}_{2}(5 \mathrm{~mL})$, and $\mathrm{BF}_{3} \cdot \mathrm{OEt}_{2}(0.210 \mathrm{~g}, 1.48 \mathrm{mmol})$. Purification by flash chromatography (2:98 EtOAc:hexanes) afforded $\mathbf{S}-5$ as a colorless oil $(0.340 \mathrm{~g}, 68 \%)$ : GC $\mathrm{t}_{\mathrm{R}} 15.1 \mathrm{~min}\left(50{ }^{\circ} \mathrm{C}, 10^{\circ} \mathrm{C} / \mathrm{min}\right)$; ${ }^{1} \mathrm{H} \mathrm{NMR}$ $\left(500 \mathrm{MHz}, \mathrm{CDCl}_{3}\right) \delta 7.54(\mathrm{~m}, 2 \mathrm{H}), 7.37(\mathrm{~m}, 3 \mathrm{H}), 6.11(\mathrm{dd}, J=18.6,4.8 \mathrm{~Hz}, 1 \mathrm{H}), 5.92(\mathrm{dd}, J=18.6,1.3 \mathrm{~Hz}, 1 \mathrm{H}), 4.30(\mathrm{~m}, 1 \mathrm{H}), 1.65$ (septet, $J=6.8 \mathrm{~Hz}, 1 \mathrm{H}), 1.21(\mathrm{~d}, J=6.4 \mathrm{~Hz}, 3 \mathrm{H}), 0.90(\mathrm{~s}, 3 \mathrm{H}), 0.89(\mathrm{~s}, 3 \mathrm{H}), 0.86(\mathrm{~s}, 6 \mathrm{H}), 0.33(\mathrm{~s}, 6 \mathrm{H}), 0.10(\mathrm{~s}, 3 \mathrm{H}), 0.08(\mathrm{~s}, 3 \mathrm{H}) ;{ }^{13} \mathrm{C}$ NMR $\left(125 \mathrm{MHz}, \mathrm{CDCl}_{3}\right) \delta 152.5,139.0,133.8,128.9,127.7,124.6,71.3,34.1,31.7,25.1,24.0,22.7,20.4,18.6,14.2,-2.4,-2.5$, -2.7; IR (thin film) 2958, 1250, $829 \mathrm{~cm}^{-1}$; HRMS (ESI) $\mathrm{m} / \mathrm{z}$ calcd for $\mathrm{C}_{20} \mathrm{H}_{36} \mathrm{OSi}_{2} \mathrm{Na}(\mathrm{M}+\mathrm{Na})^{+} 371.2202$, found 371.2205 .
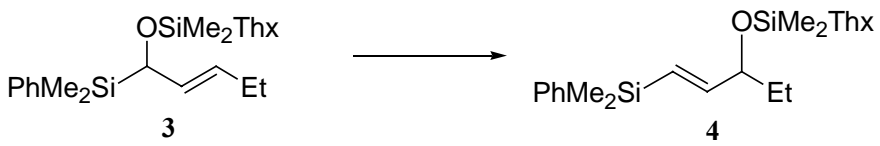

$\alpha$-Silyloxy Vinylic Silane 4. As above the representative procedure for $\alpha$-silyloxy allylic silane rearrangement was followed using $\alpha$ silyloxy allylic silane $(0.363 \mathrm{~g}, 1.00 \mathrm{mmol}), \mathrm{CH}_{2} \mathrm{Cl}_{2}(4 \mathrm{~mL})$, and $\mathrm{BF}_{3} \cdot \mathrm{OEt}_{2}(0.147 \mathrm{~g}, 1.04 \mathrm{mmol})$. Purification by flash chromatography (2:98 EtOAc:hexanes) afforded 4 as a colorless oil $(0.149 \mathrm{~g}, 41 \%)$ : $\mathrm{GC} \mathrm{t}_{\mathrm{R}} 13.7 \mathrm{~min}\left(50{ }^{\circ} \mathrm{C}, 10{ }^{\circ} \mathrm{C} / \mathrm{min}\right)$; ${ }^{1} \mathrm{H} \mathrm{NMR}$ $\left(500 \mathrm{MHz}, \mathrm{CDCl}_{3}\right) \delta 7.54(\mathrm{~m}, 2 \mathrm{H}), 7.37(\mathrm{~m}, 3 \mathrm{H}), 6.05(\mathrm{dd}, J=18.7,5.6 \mathrm{~Hz}, 1 \mathrm{H}), 5.89(\mathrm{dd}, \mathrm{J}=18.7,1.0 \mathrm{~Hz}, 1 \mathrm{H}), 4.09(\mathrm{~m}, 1 \mathrm{H}), 1.68$ (septet, $J=6.8 \mathrm{~Hz}, 1 \mathrm{H}), 1.50(\mathrm{~m}, 2 \mathrm{H}), 0.89(\mathrm{~d}, J=6.8 \mathrm{~Hz}, 6 \mathrm{H}), 0.86(\mathrm{~m}, 3 \mathrm{H}), 0.84(\mathrm{~s}, 6 \mathrm{H}), 0.34(\mathrm{~s}, 6 \mathrm{H}), 0.09(\mathrm{~s}, 3 \mathrm{H}), 0.06(\mathrm{~s}, 3 \mathrm{H}) ;{ }^{13} \mathrm{C}$ NMR $\left(125 \mathrm{MHz}, \mathrm{CDCl}_{3}\right) \delta 151.6,139.2,134.1,129.1,127.9,126.3,77.0,34.4,30.8,25.3,20.6,18.9,9.9,-2.0,-2.2,-2.3,-2.5 ;$ IR (thin film) 2959, 1250, $831 \mathrm{~cm}^{-1}$; HRMS (ESI) $\mathrm{m} / \mathrm{z}$ calcd for $\mathrm{C}_{21} \mathrm{H}_{38} \mathrm{OSi}_{2} \mathrm{Na}(\mathrm{M}+\mathrm{Na})^{+}$385.2359, found 385.2360. Anal. Calcd for $\mathrm{C}_{18} \mathrm{H}_{32} \mathrm{OSi}_{2}$ : C, 69.54; H, 10.56. Found: C, 69.32; H, 10.40 .

\section{Rearrangement of $( \pm) \alpha$-Pivalyloxy Allylic Silanes}

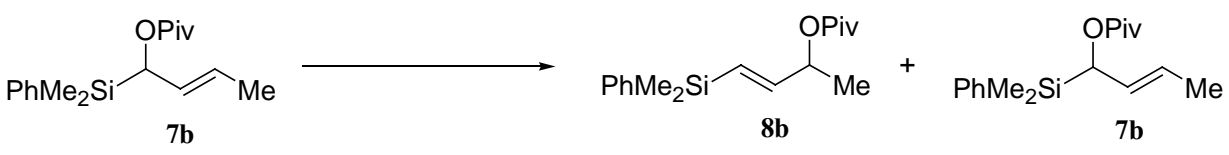

$\alpha$-Pivalyloxy Vinylic Silane 8b. The representative procedure for $\alpha$-silyloxy allylic silane rearrangement was followed using $\alpha$ pivalyloxy allylic silane $(0.200 \mathrm{~g}, 0.69 \mathrm{mmol}), \mathrm{CH}_{2} \mathrm{Cl}_{2}(5 \mathrm{~mL})$, and $\mathrm{BF}_{3} \cdot \mathrm{OEt}_{2}(0.108 \mathrm{~g}, 0.75 \mathrm{mmol})$. GC analysis of the unpurified product revealed an incomplete rearrangement. (20\% completion). 


\section{Crossover Experiment of Rearrangement}

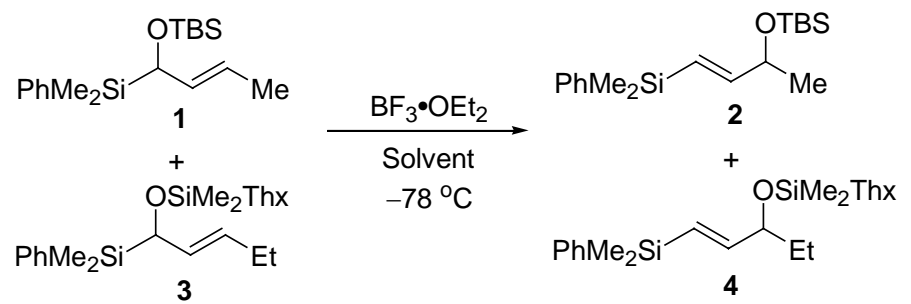

Crossover Rearrangement. To a cooled solution $\left(-78^{\circ} \mathrm{C}\right)$ of $\alpha$-silyloxy allylic silane $\mathbf{1}(0.177 \mathrm{~g}, 0.551 \mathrm{mmol})$ and $\alpha$-silyloxy allylic silane $3(0.200 \mathrm{~g}, 0.551 \mathrm{mmol})$ in $4 \mathrm{~mL}$ of solvent was added $\mathrm{BF}_{3} \cdot \mathrm{OEt}_{2}(0.162 \mathrm{~g}, 1.14 \mathrm{mmol})$. After $3 \mathrm{~h} \mathrm{at}-78^{\circ} \mathrm{C}$, an aqueous solution of $\mathrm{NaHCO}_{3}(15 \mathrm{~mL})$ was added. The resultant layers were separated and the aqueous layer was extracted with $3 \times 5 \mathrm{~mL}$ of hexanes. The combined organic layers were washed with saturated aqueous $\mathrm{NaCl}(15 \mathrm{~mL})$, dried over $\mathrm{MgSO}_{4}$, and filtered. GC analysis revealed the formation of vinylic silanes $\mathbf{2}$ and $\mathbf{4}$ exclusively.

\section{Stereochemistry Scrambling Experiments}

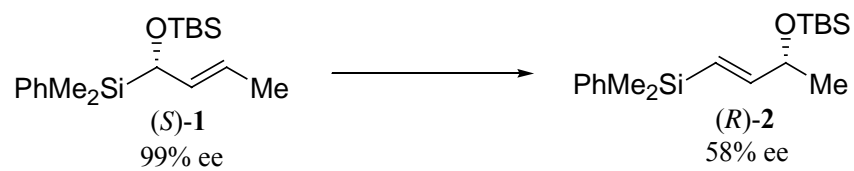

$\alpha$-Silyloxy Allylic Silane Rearrangement. To a cooled $\left(-78^{\circ} \mathrm{C}\right)$ solution of $\alpha$-silyloxy allylic silane $(0.044 \mathrm{~g}, 0.137 \mathrm{mmol})$ in $0.5 \mathrm{~mL}$ of $\mathrm{CH}_{2} \mathrm{Cl}_{2}$ was added $\mathrm{BF}_{3} \cdot \mathrm{OEt}_{2}(0.018 \mathrm{~g}, 0.125 \mathrm{mmol})$. After $3 \mathrm{~h}$ at $-78^{\circ} \mathrm{C}$, an aqueous solution of $\mathrm{NaHCO}_{3}(10 \mathrm{~mL})$ was added. The resultant layers were separated and the aqueous layer was extracted with $3 \times 5 \mathrm{~mL}$ of hexanes. The combined organic layers were washed with saturated aqueous $\mathrm{NaCl}(10 \mathrm{~mL})$, dried over $\mathrm{MgSO}_{4}$, and filtered. The filtrate was concentrated in vacuo to afford the $\alpha$ silyloxy vinylic silane as a light yellow oil. Vinylic silane $(R)-2$ was purified by silica gel chromatography (2:98 EtOAc:hexanes) (89\%). Deprotection of the $\alpha$-silyloxy allylic silane using the representative deprotection procedure (vide infra) afforded $\alpha$-hydroxy vinylic silane (48\%), which was analyzed by chiral HPLC (Chiralcel OD-H column, 98:2 hexanes / IPA, $1 \mathrm{~mL} / \mathrm{min}, 254 \mathrm{~nm}$ ) and found to be $58 \%$ ee. The product was identical to ( \pm )-S-7 by ${ }^{1} \mathrm{H}$ NMR and ${ }^{13} \mathrm{C}$ NMR spectroscopic analysis (vide infra).

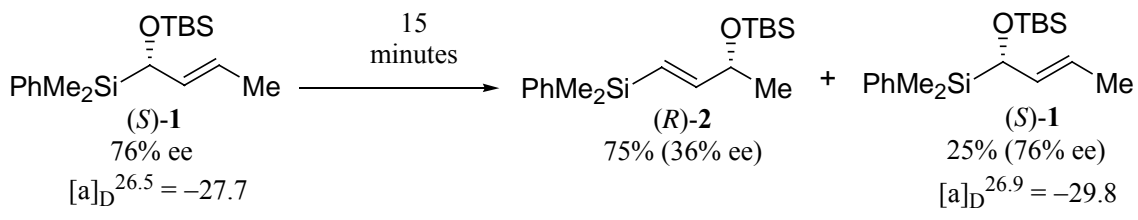

Control Experiment to Determine the Enantiomeric Excess of Recovered Starting Material: To a cooled solution $\left(-78^{\circ} \mathrm{C}\right)$ of $\alpha$ silyloxy allylic silane $(0.300 \mathrm{~g}, 0.936 \mathrm{mmol})$ in $3.5 \mathrm{~mL}$ of $\mathrm{CH}_{2} \mathrm{Cl}_{2}$ was added $\mathrm{BF}_{3} \cdot \mathrm{OEt}_{2}(0.133 \mathrm{~g}, 0.936 \mathrm{mmol})$. After $3 \mathrm{~h}$ at $-78^{\circ} \mathrm{C}$, an aqueous solution of $\mathrm{NaHCO}_{3}(30 \mathrm{~mL})$ was added. The resultant layers were separated and the aqueous layer was extracted with $3 \times$ $15 \mathrm{~mL}$ of hexanes. The combined organic layers were washed with saturated aqueous $\mathrm{NaCl}(30 \mathrm{~mL})$, dried over $\mathrm{MgSO}_{4}$, and filtered. The filtrate was concentrated in vacuo to afford the $\alpha$-silyloxy vinylic silane as a light yellow oil. Vinylic silane $(R)$ - 2 and allylic silane (S)-1 were purified by silica gel chromatography (hexanes to 2:98 EtOAc:hexanes). Recovered (S)-1 was identical to ( \pm )-1 by ${ }^{1} \mathrm{H}$ NMR and ${ }^{13} \mathrm{C}$ NMR spectroscopic analysis (vide supra): $[\alpha]_{\mathrm{D}}^{20}-29.8$ (c 1.0, $\mathrm{CHCl}_{3}$ ). Deprotection of the $\alpha$-silyloxy allylic silane using the representative deprotection procedure (vide infra) afforded $\alpha$-hydroxy vinylic silane (73\%), which was analyzed by chiral HPLC (Chiralcel OD-H column, 98:2 hexanes / IPA, $1 \mathrm{~mL} / \mathrm{min}, 254 \mathrm{~nm}$.) and found to have $36 \%$ ee. The product was identical to $( \pm)-\mathrm{S}-7$ by ${ }^{1} \mathrm{H}$ NMR and ${ }^{13} \mathrm{C}$ NMR spectroscopic analysis (vide infra): $[\alpha]^{20}{ }_{\mathrm{D}}-1.7\left(c 1.03, \mathrm{CHCl}_{3}\right)$.

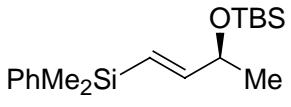

(S)-2 $95 \%$ ee<smiles>CCCCCC[SiH2]C=CC(C)[OH2+]</smiles>

(S)-2

95\% eе

Control experiment to Determine Stereochemical Stability of Product. To a cooled solution $\left(-78{ }^{\circ} \mathrm{C}\right)$ of $\alpha$-silyloxy allylic silane $(0.583 \mathrm{~g}, 1.82 \mathrm{mmol})$ in $7 \mathrm{~mL}$ of $\mathrm{CH}_{2} \mathrm{Cl}_{2}$ was added $\mathrm{BF}_{3} \cdot \mathrm{OEt}_{2}(0.289 \mathrm{~g}, 2.03 \mathrm{mmol})$. After 3 hours at $-78^{\circ} \mathrm{C}$ an aqueous solution of $\mathrm{NaHCO}_{3}(50 \mathrm{~mL})$ was added. The resultant layers were separated and the aqueous layer was re-extracted with $3 \times 25 \mathrm{~mL}$ of hexanes. The combined organic layers were washed with saturated aqueous $\mathrm{NaCl}(30 \mathrm{~mL})$, dried over $\mathrm{MgSO}_{4}$ and filtered. The filtrate was concentrated in vacuo to afford the $\alpha$-silyloxy vinylic silane as a light yellow oil. Vinylic silane $(S)-2$ was purified by silica gel 
chromatography (2:98 EtOAc:hexanes) (89\%). Deprotection of the $\alpha$-silyloxy allylic silane using the representative deprotection procedure afforded $\alpha$-hydroxy vinylic silane (91\%), which was analyzed by chiral HPLC (Chiralcel OD-H column, 98:2 hexanes / IPA, $1 \mathrm{~mL} / \mathrm{min}, 254 \mathrm{~nm}$.) and found to have $95 \%$ enantiomeric excess. The product was identical to $( \pm)-\mathrm{S}-7$ by ${ }^{1} \mathrm{H}$ NMR and ${ }^{13} \mathrm{C}$ NMR spectroscopic analysis (vide infra): $[\alpha]^{20} 6.3\left(\right.$ c $\left.1.05, \mathrm{CHCl}_{3}\right)$.

\section{Deprotection of $\alpha$-Silyloxy Vinylic Silanes}
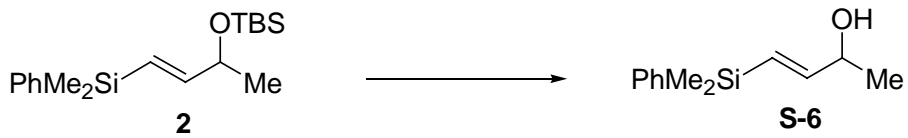

Procedure for Deprotection of $\boldsymbol{\alpha}$-Silyloxy Vinylic Silane S-6. To a solution of $\alpha$-silyloxy vinylic silane (0.461 g, 1.44 mmol) in 5 $\mathrm{mL}$ of THF at $23{ }^{\circ} \mathrm{C}$ was added tetrabutylammonium fluoride $\left(2.88 \mathrm{~mL}, 2.9 \mathrm{mmol}, 1.0 \mathrm{M}\right.$ solution in THF). After $18 \mathrm{~h}$ at $23{ }^{\circ} \mathrm{C}$, a saturated aqueous solution of ammonium chloride $(50 \mathrm{~mL})$ was added. The resultant layers were separated and the aqueous layer was

extracted with $(3 \times 25 \mathrm{~mL})$ of diethyl ether. The combined organic layers were washed with saturated aqueous $\mathrm{NaCl}(30 \mathrm{~mL})$, dried over $\mathrm{MgSO}_{4}$, and filtered. The filtrate was concentrated in vacuo to afford the $\alpha$-hydroxy vinylic silane as a light yellow oil. Purification by flash chromatography (10:90 EtOAc:hexanes) afforded S-6 as a colorless oil (0.269 $\mathrm{g}, 91 \%)$ : GC $\mathrm{t}_{\mathrm{R}} 9.3 \mathrm{~min}\left(50{ }^{\circ} \mathrm{C}, 10\right.$ $\left.{ }^{\circ} \mathrm{C} / \mathrm{min}\right) ;{ }^{1} \mathrm{H} \mathrm{NMR}\left(500 \mathrm{MHz}, \mathrm{CDCl}_{3}\right) \delta 7.52(\mathrm{~m}, 2 \mathrm{H}), 7.36(\mathrm{~m}, 3 \mathrm{H}), 6.17(\mathrm{dd}, J=18.7,4.9 \mathrm{~Hz}, 1 \mathrm{H}), 5.97(\mathrm{~d}, J=18.7,1.5 \mathrm{~Hz}, 1 \mathrm{H})$, $4.32(\mathrm{~m}, 1 \mathrm{H}), 1.55$ (br s, $1 \mathrm{H}) 1.28(\mathrm{~d}, J=6.4 \mathrm{~Hz}, 3 \mathrm{H}), 0.35(\mathrm{~s}, 6 \mathrm{H}) ;{ }^{13} \mathrm{C} \mathrm{NMR}\left(125 \mathrm{MHz}, \mathrm{CDCl}_{3}\right) \delta 151.4,138.5,133.9,129.1,127.9$, 126.0, 70.5, 23.0, -2.6; IR (thin film) 3338, 2961, 1248, $825 \mathrm{~cm}^{-1}$; HRMS (ESI) $\mathrm{m} / \mathrm{z}$ calcd for $\mathrm{C}_{12} \mathrm{H}_{18} \mathrm{OSiNa}_{(\mathrm{M}+\mathrm{Na}}{ }^{+} 229.1025$, found 229.1017. Anal. Calcd for $\mathrm{C}_{12} \mathrm{H}_{18} \mathrm{OSi}$ : C, 69.84; H, 8.79. Found: C, 69.51; H, 8.88.

\section{References}

(1) Pangborn, A. B.; Giardello, M. A.; Grubbs, R. H.; Rosen, R. K.; Timmers, F. J. Organometallics 1996, 15, $1518-1520$.

(2) Ager, D. J.; Fleming, I.; Patel, S. K. J. Chem. Soc., Perkin Trans. 1 1981, 2520-2526.

(3) Takeda, K.; Ohnishi, Y.; Koizumi, T. Org. Lett. 1999, 1, 237-239.

(4) Sakaguchi, K.; Higashino, M.; Ohfune, Y. Tetrahedron 2003, 59, 6647-6658.

\section{Spectral Data}




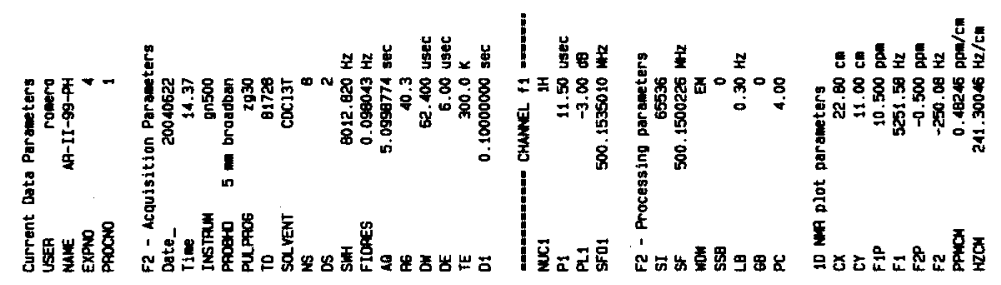

c9lt

SEEO 0

2082. 0

2082

osere: 0

2688.0

$\begin{array}{lll}9106 & 0\end{array}$

L659' 1

$2599 \cdot 1$

orcas

roL9?

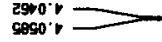

$\angle a t r s$

ootr's

gier $s$

orgr $\mathrm{s}$

EEgr $\mathrm{s}$

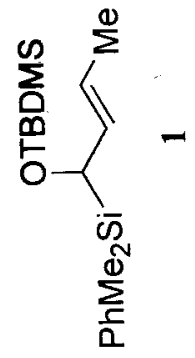

IoLe

EGEE: 2

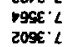

$\begin{array}{ll}2055: & L \\ 2095 & L\end{array}$

$629 \mathrm{C} L$

9Ser: $\angle$

EESS.

Ergs

0L95' $\angle$

\section{.}




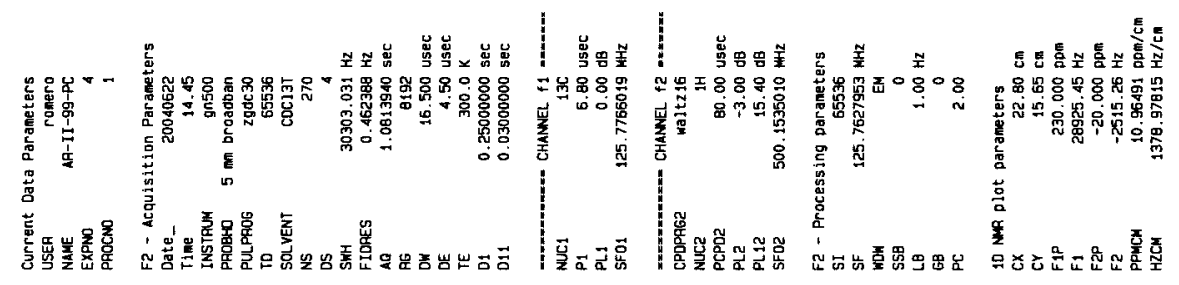

co. $\mathrm{s}-$

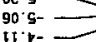

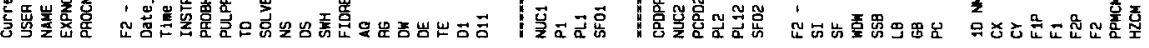

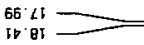

EI ' 92

E' 89

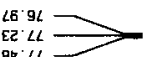

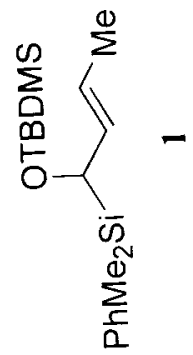

เ2' 225

49625

${ }_{41}^{262} \cdot 62=$

घi' 26

99 हㄷ

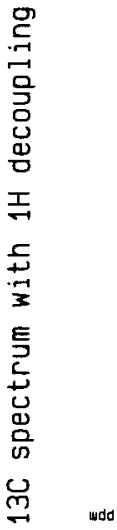



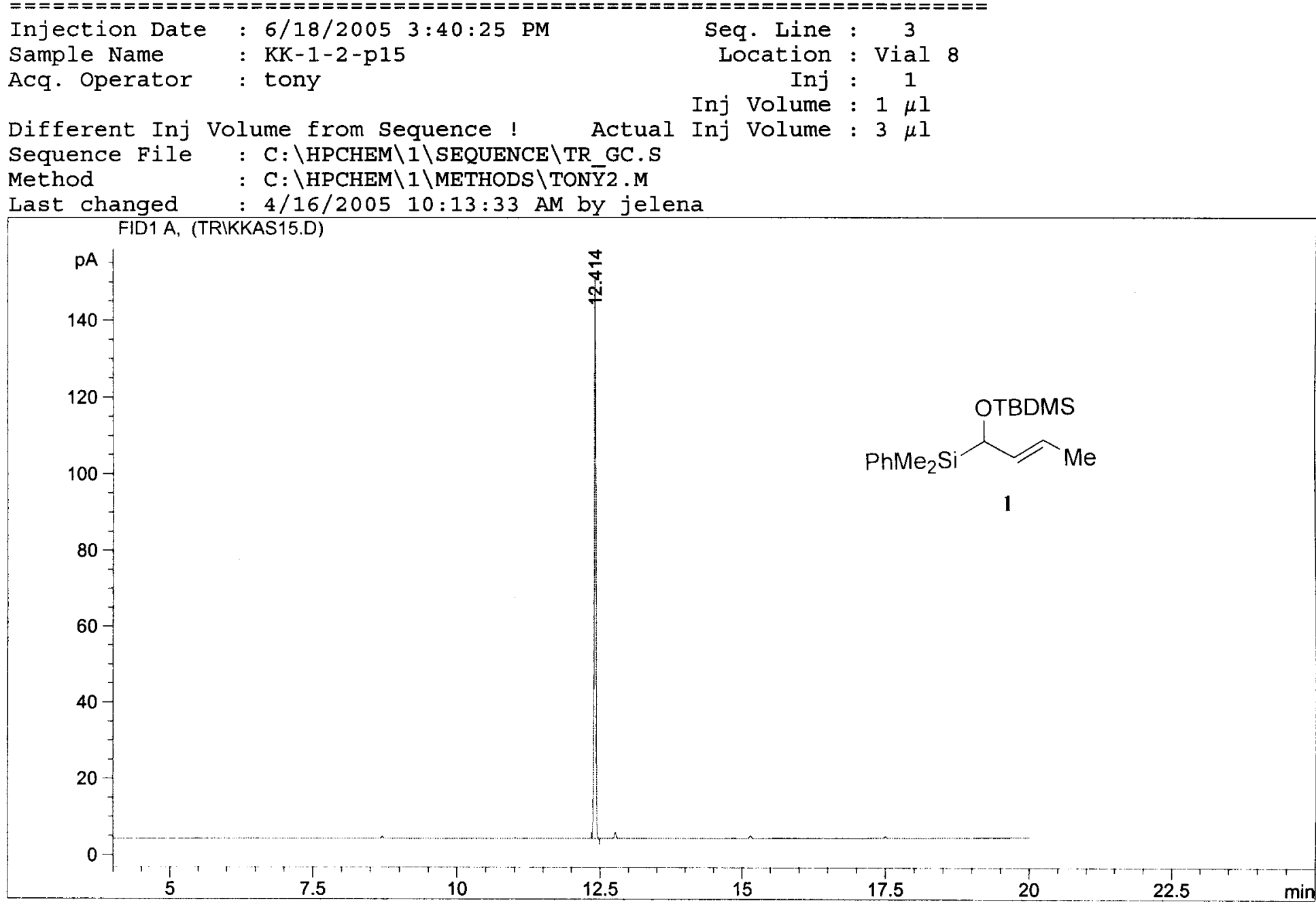

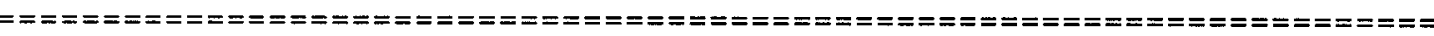

Area Percent Report

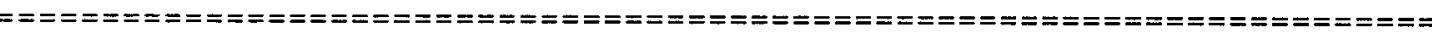

Sorted By

Multiplier

Signal

Dilution

1.0000

1.0000

Signal 1: FID1 A,

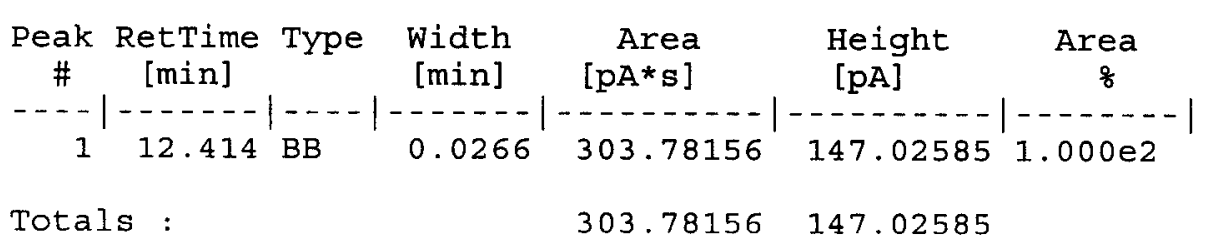

Results obtained with enhanced integrator! 

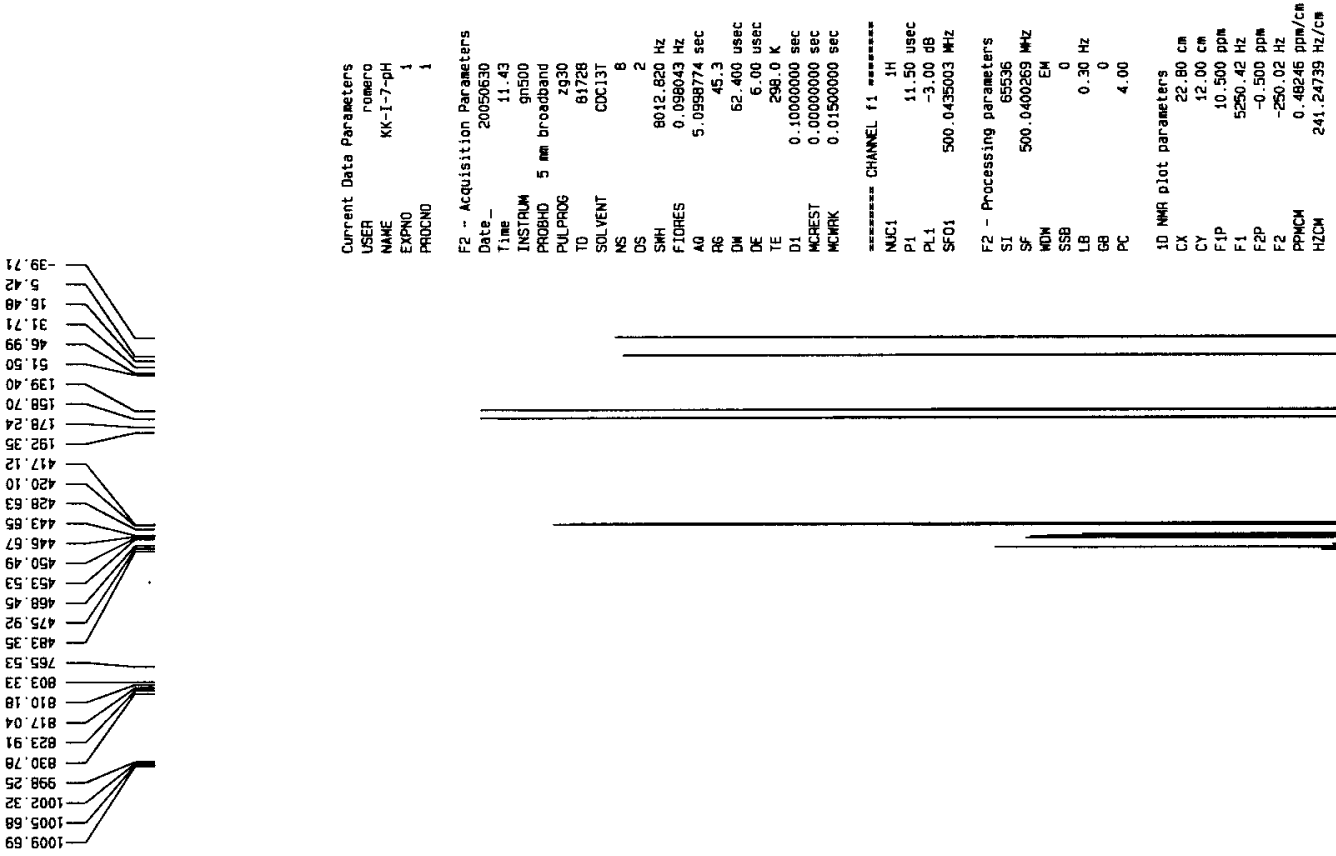

g2' 6202

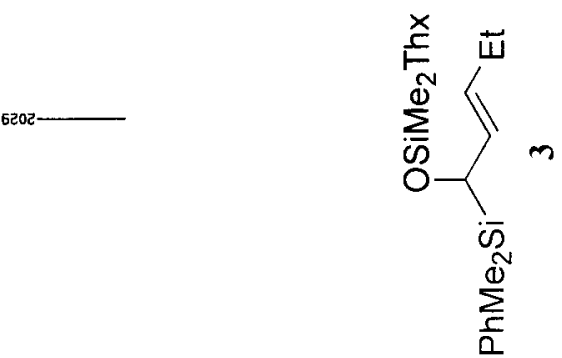

$96 \cdot 9 \mathrm{~s}<2-$

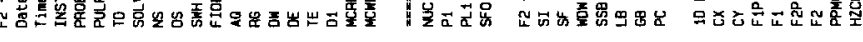

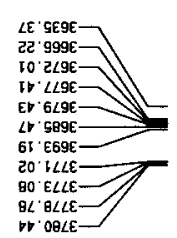

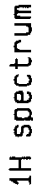
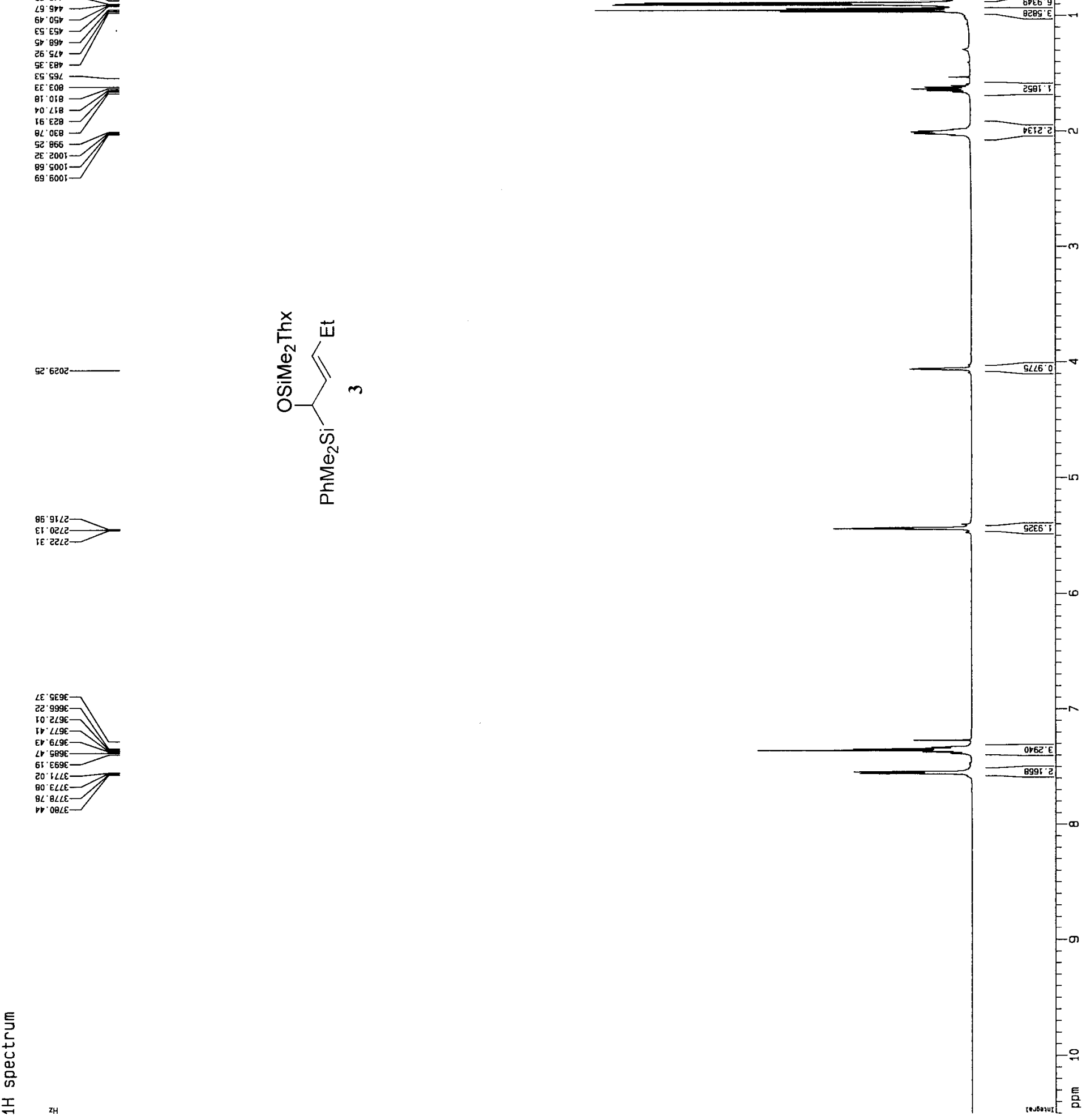

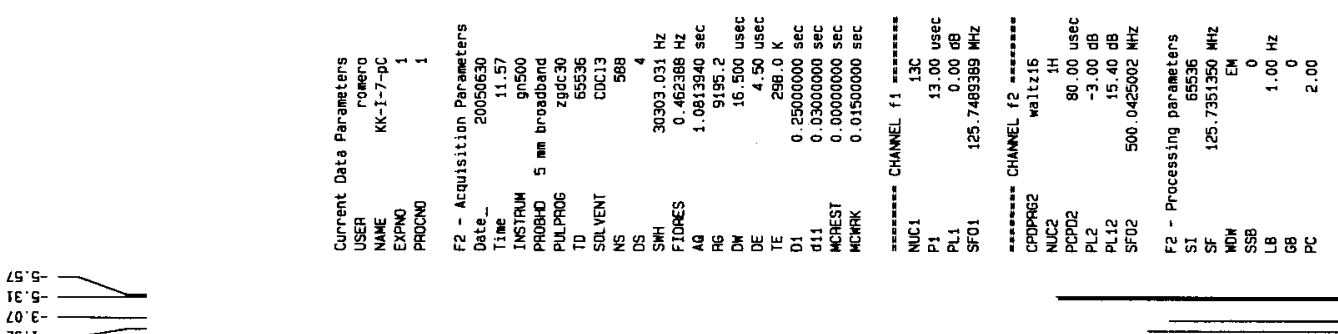

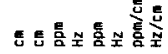

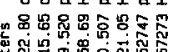

N

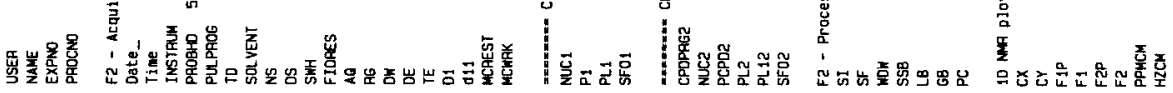
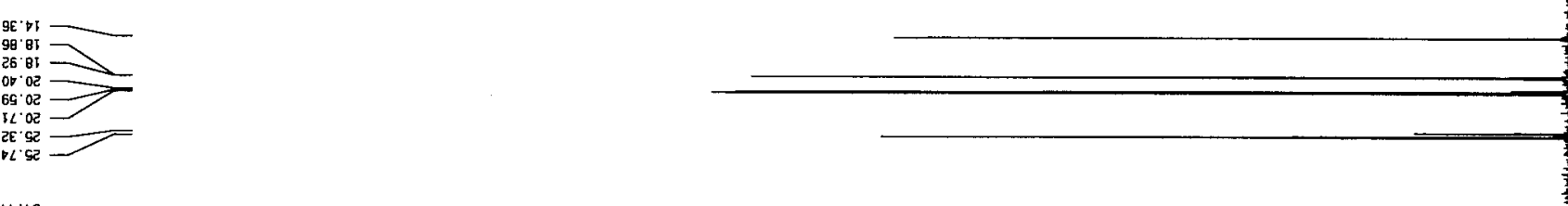

6r' 89

$\underset{\substack{66 \\ 32.42 \\ 05: 21}}{0.20}$
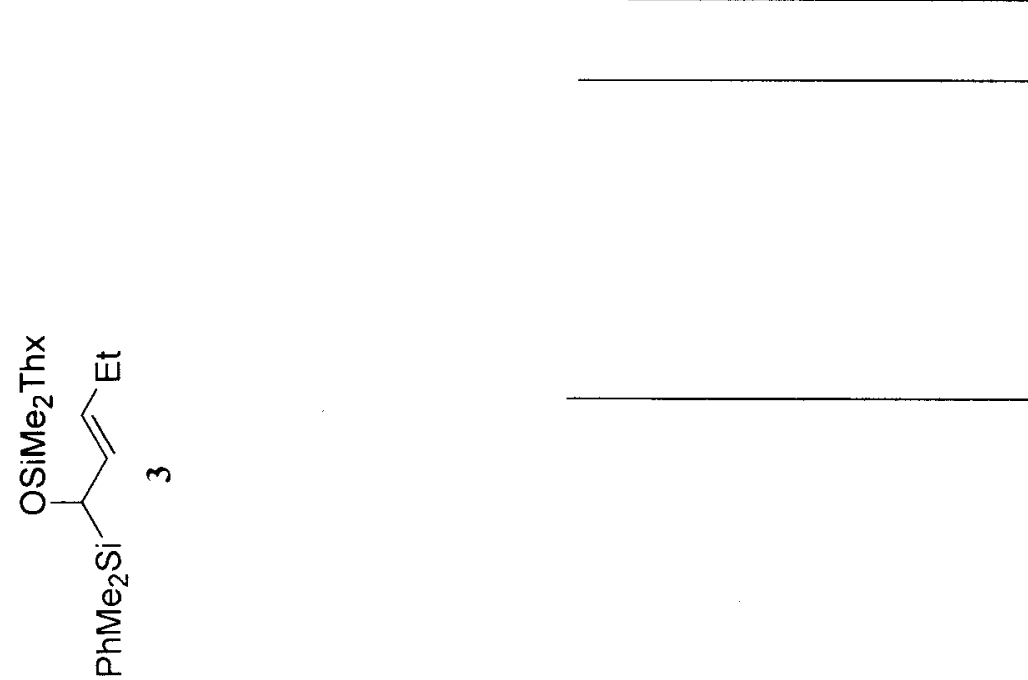

$\sum_{\frac{N}{\alpha}}^{\bar{d}}$

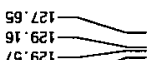

$49^{\circ} 62$

${ }_{\mathrm{BZ}} \mathrm{O} \mathrm{OES}=$

S9 $\angle E$ - 

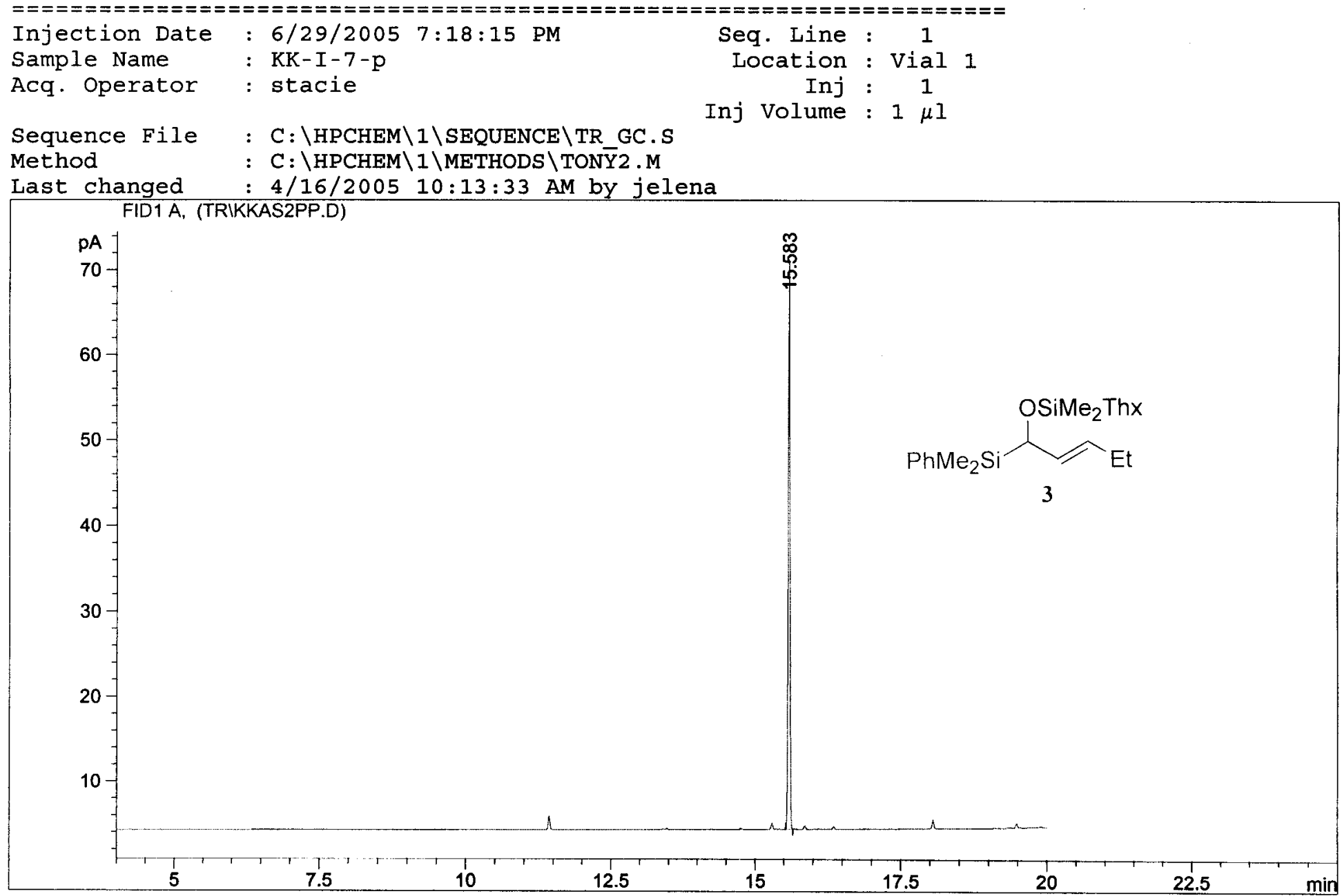

\section{Area Percent Report}

$\begin{array}{lll}\text { Sorted By } & : & \text { Signal } \\ \text { Multiplier } & : & 1.0000 \\ \text { Dilution } & : & 1.0000\end{array}$

Signal 1: FID1 A,

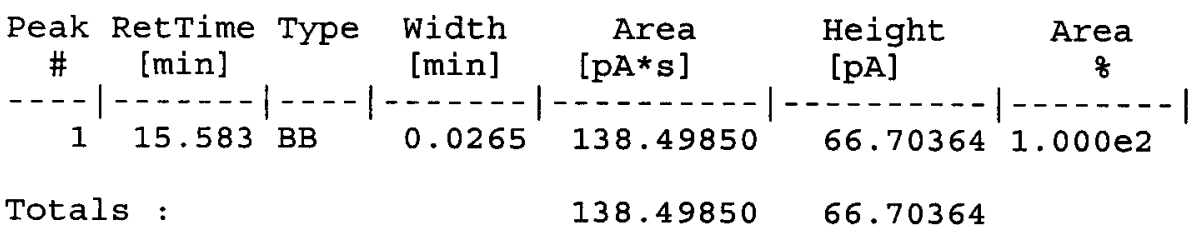

Results obtained with enhanced integrator! 


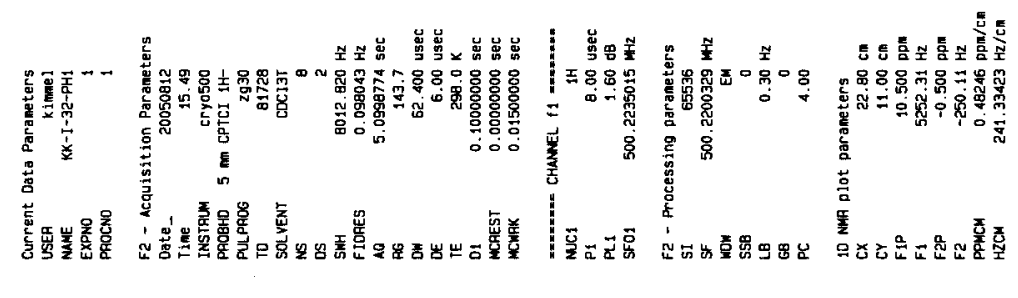

$\operatorname{sen} 0-1$

$8900 \cdot 0-$

E6r2 0

Eesg 0

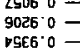

$\varepsilon \angle 46^{\prime} \mathrm{I}$

$$
\text { 政政 }
$$

2020 '

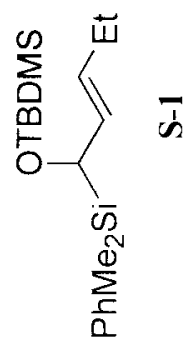

grors 
Supporting Information: Kim, Kimmel, Romero, Smitrovich, and Woerpel

$S-14$

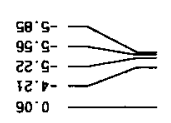

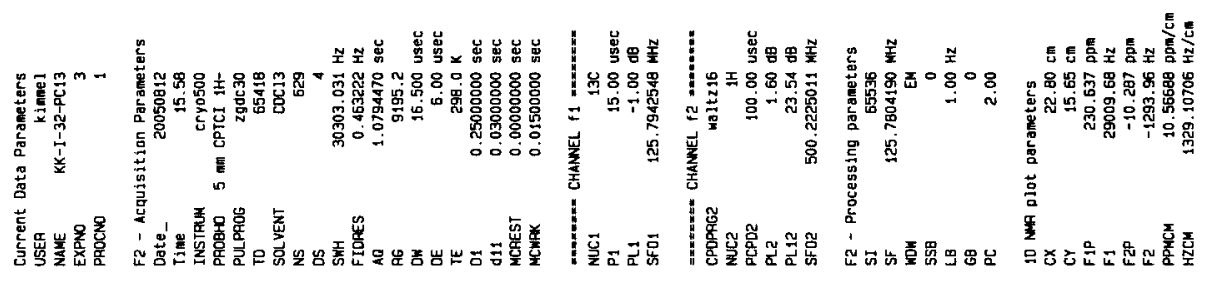

${ }^{2 \cdot} \cdot \mathrm{r} \cdot \mathrm{Br}-$

${ }_{96}^{96} \cdot 92=$
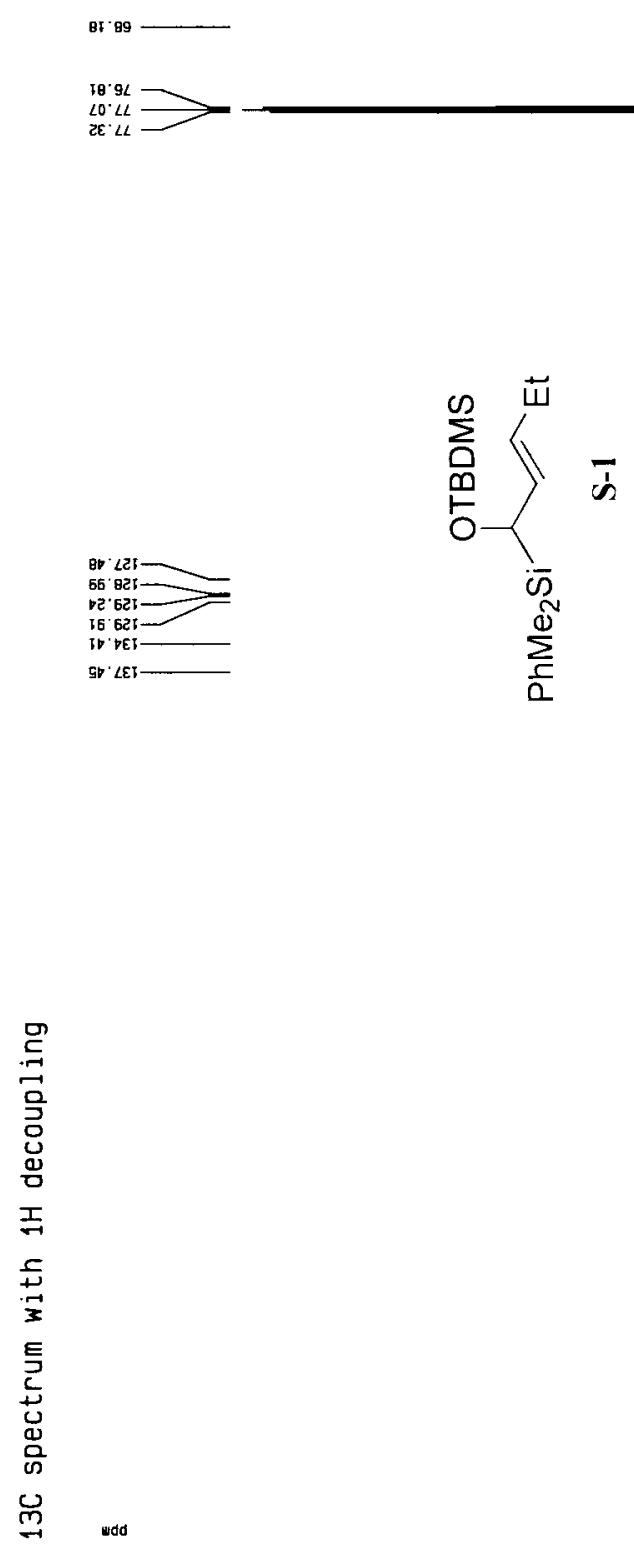

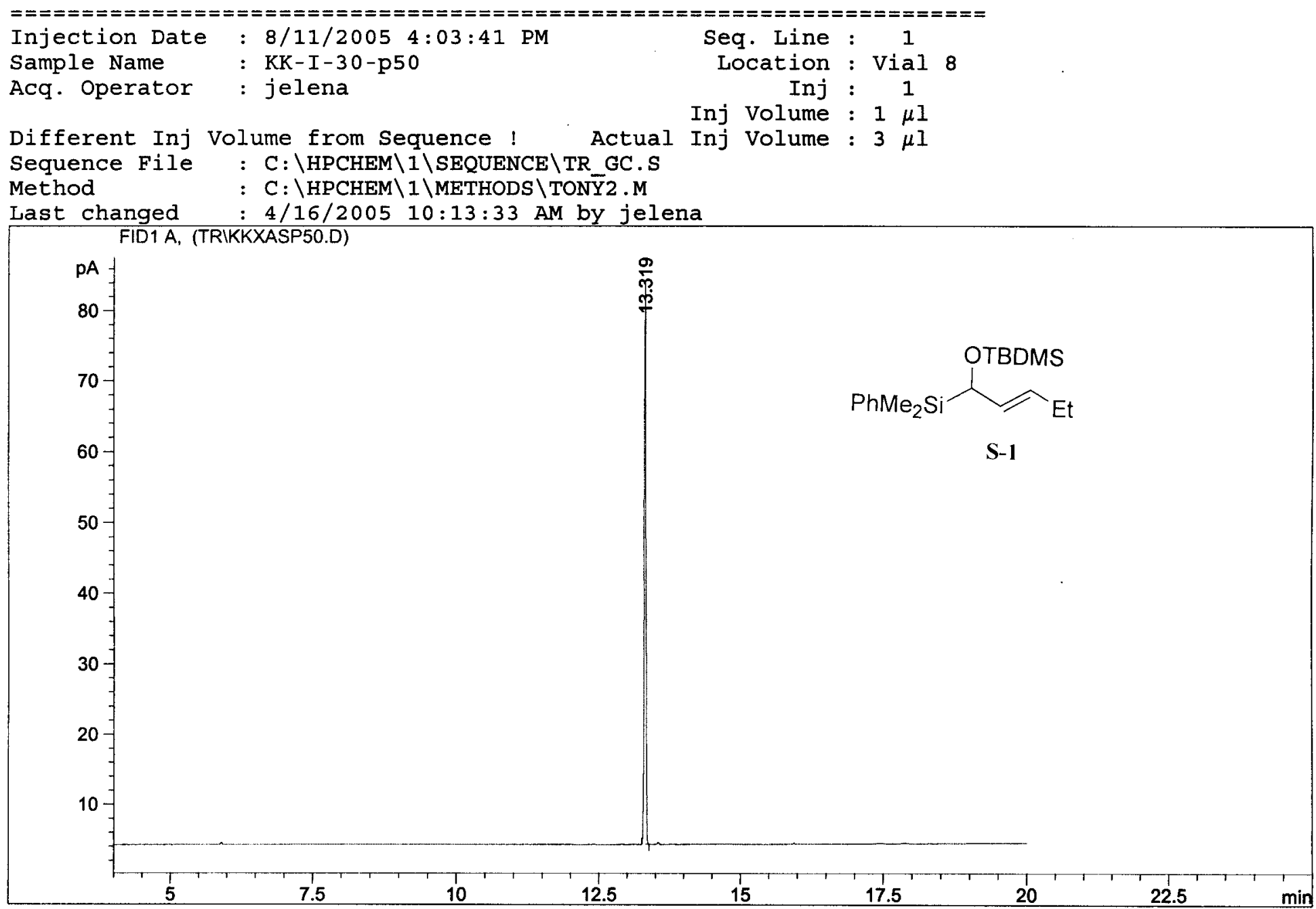

\section{Area Percent Report}

$\begin{array}{lll}\text { Sorted By } & : & \text { Signal } \\ \text { Multiplier } & : & 1.0000 \\ \text { Dilution } & : & 1.0000\end{array}$

Signal 1: FID1 A,

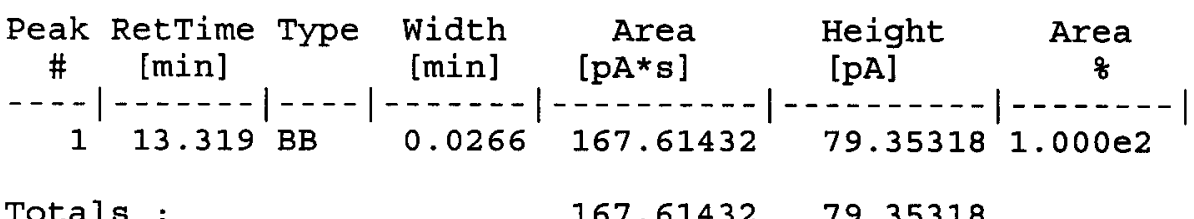

Totals :

$167.61432 \quad 79.35318$

Results obtained with enhanced integrator!

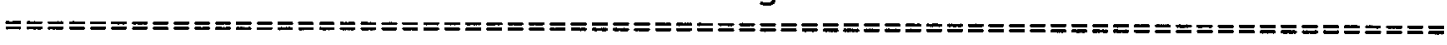

*** End of Report 


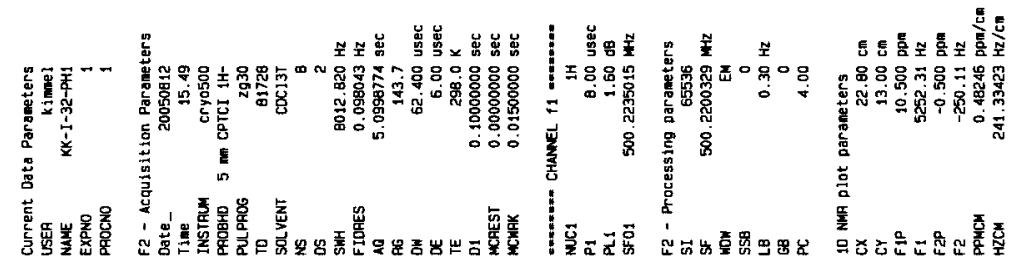

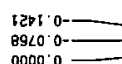

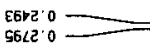

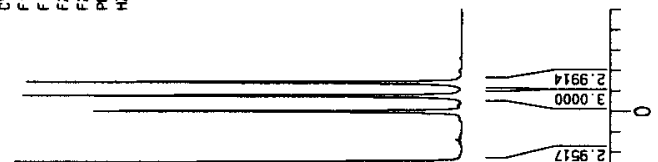

$\operatorname{eccs} \cdot 0$

$8506 \cdot 0$
$\angle 026 \cdot 0$
$\rightarrow S E 6 \cdot 0$

OGEG' 1

$\varepsilon\left\langle\mathcal{L}^{\prime} \cdot \mathrm{T}\right.$

$2020^{\circ} \mathrm{b}$

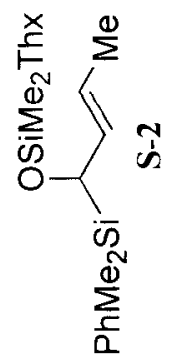

$910+5$

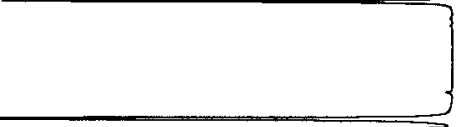

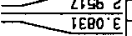

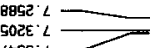

LEEE'L

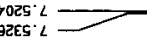
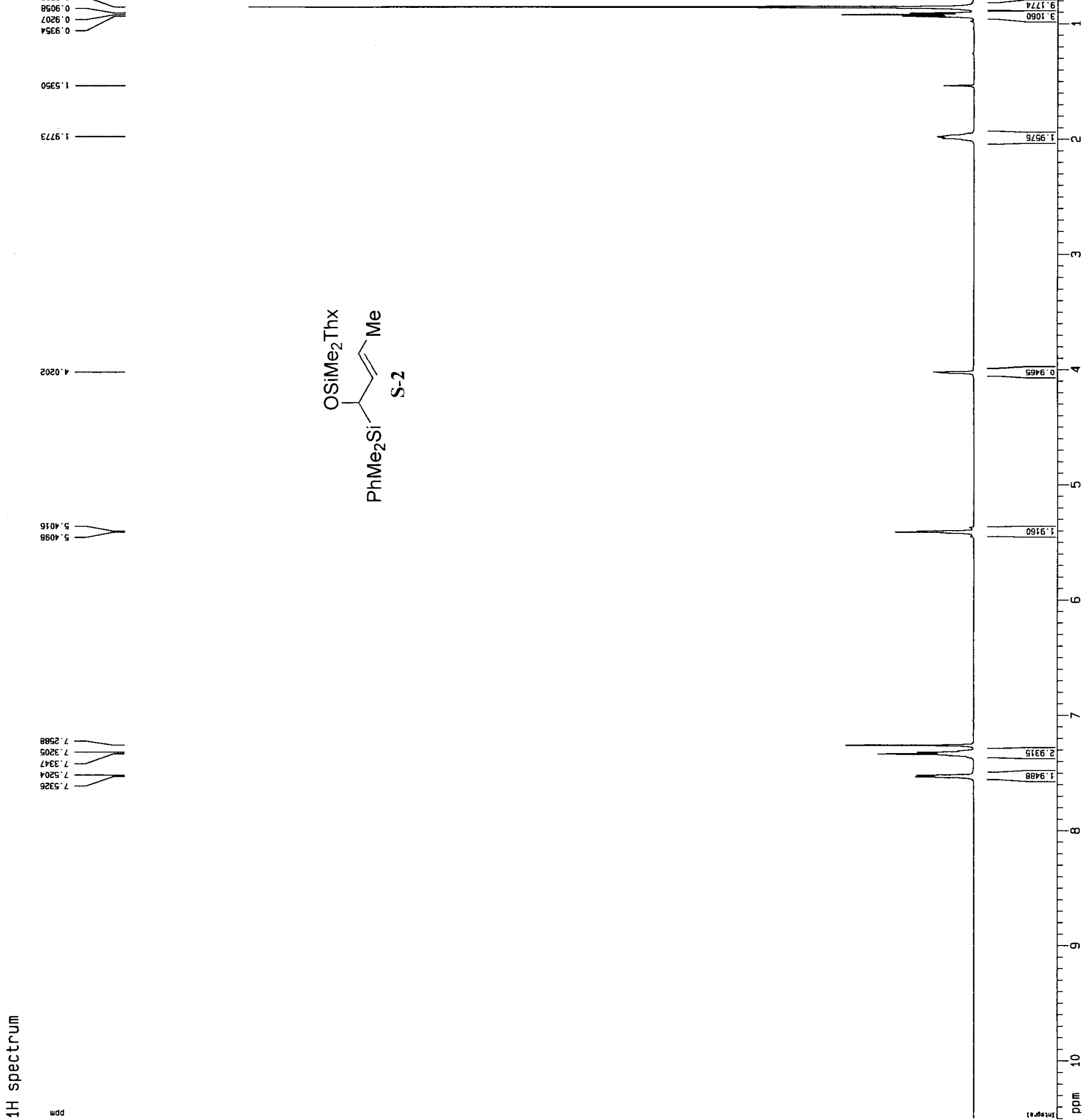


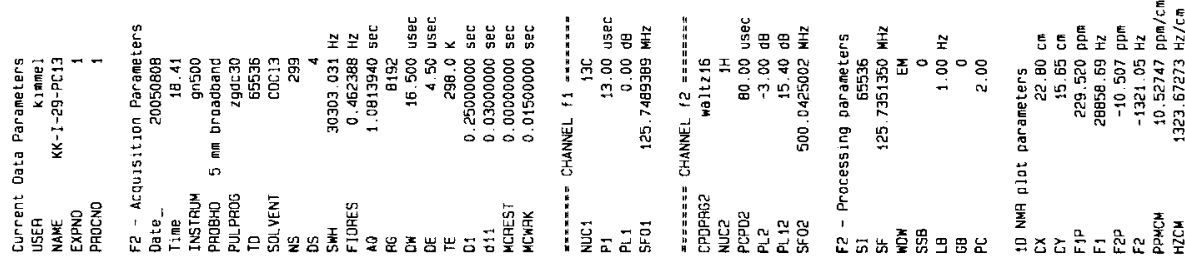

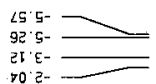

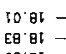

68.85

${ }_{0<}^{95} 02=$

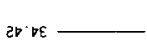

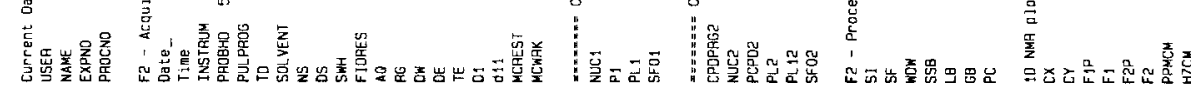
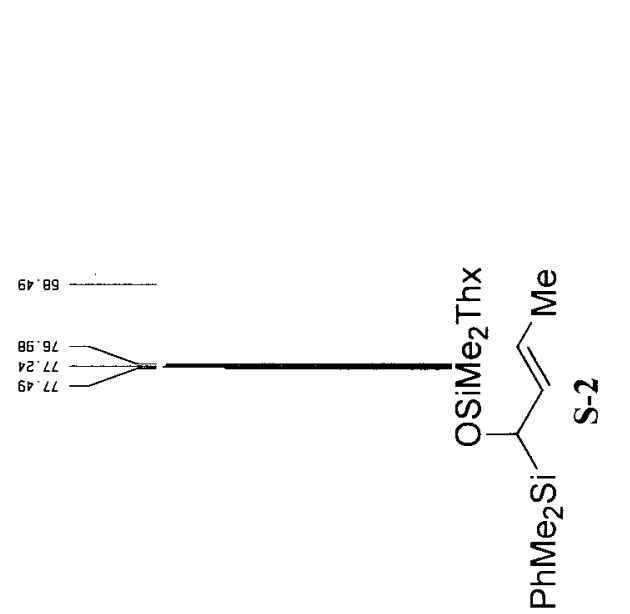

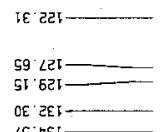

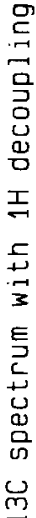

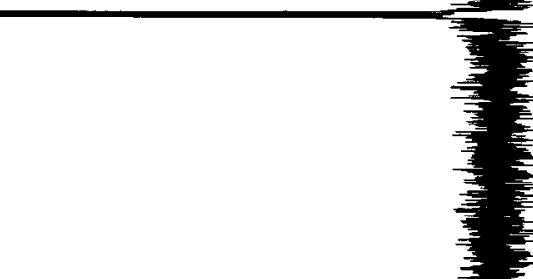



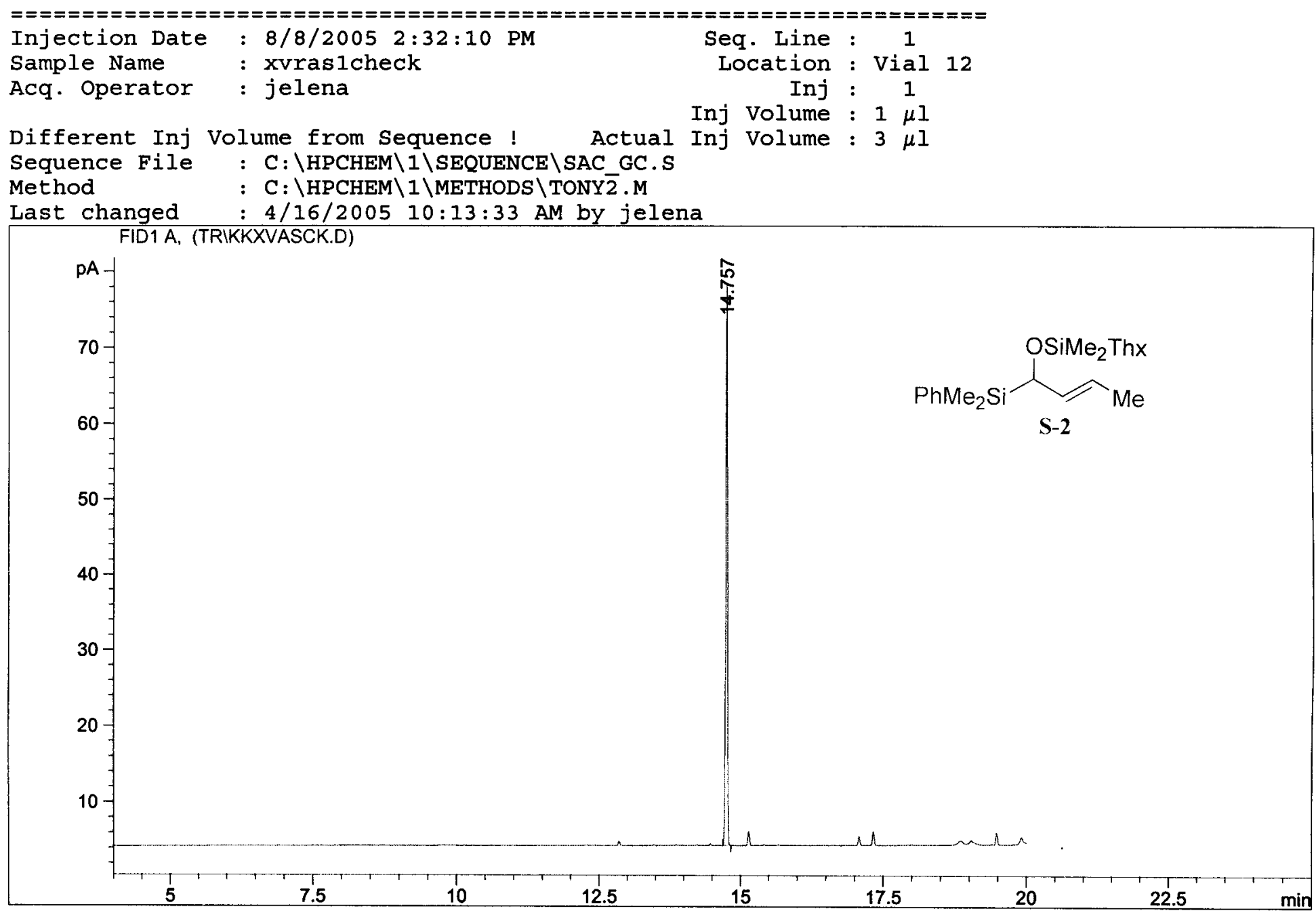

Signal 1: FID1 A,

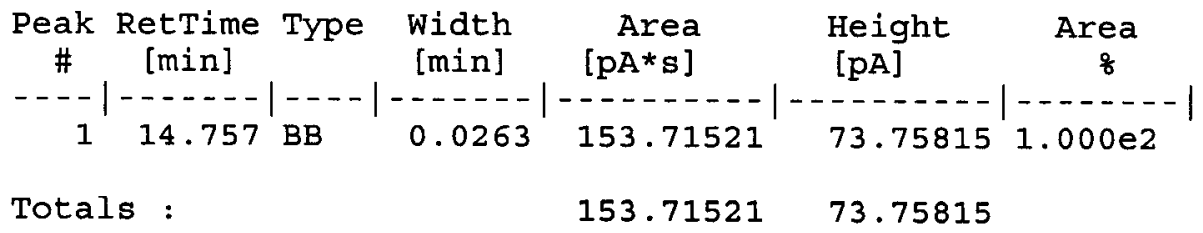

Results obtained with enhanced integrator! 

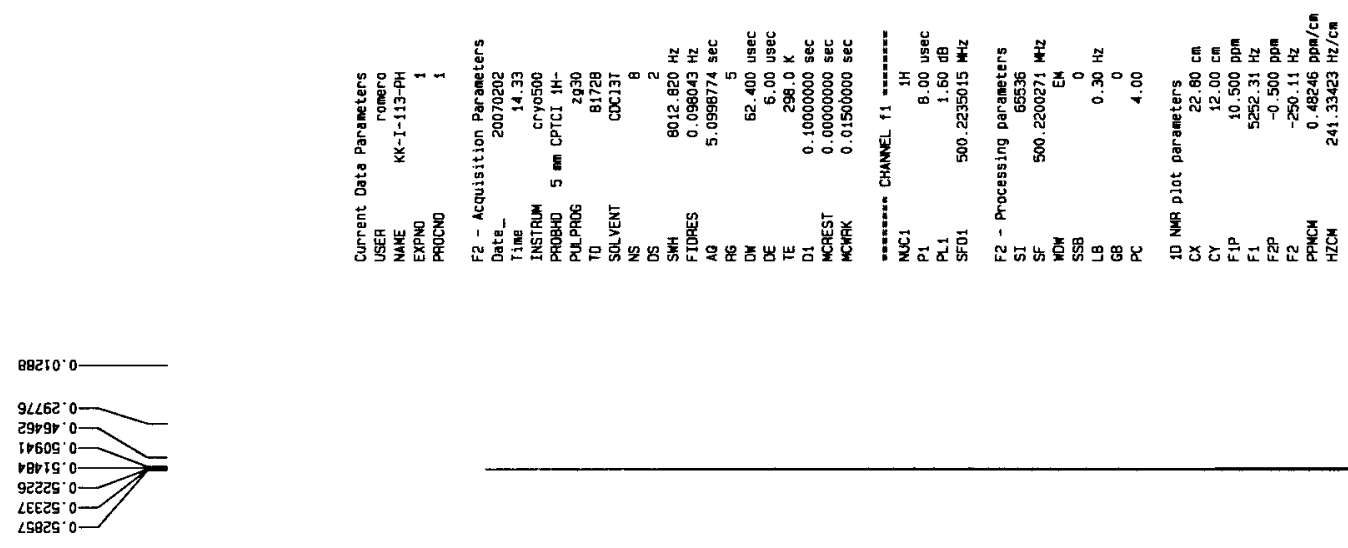

GEट58: $: 5$

rogeg,
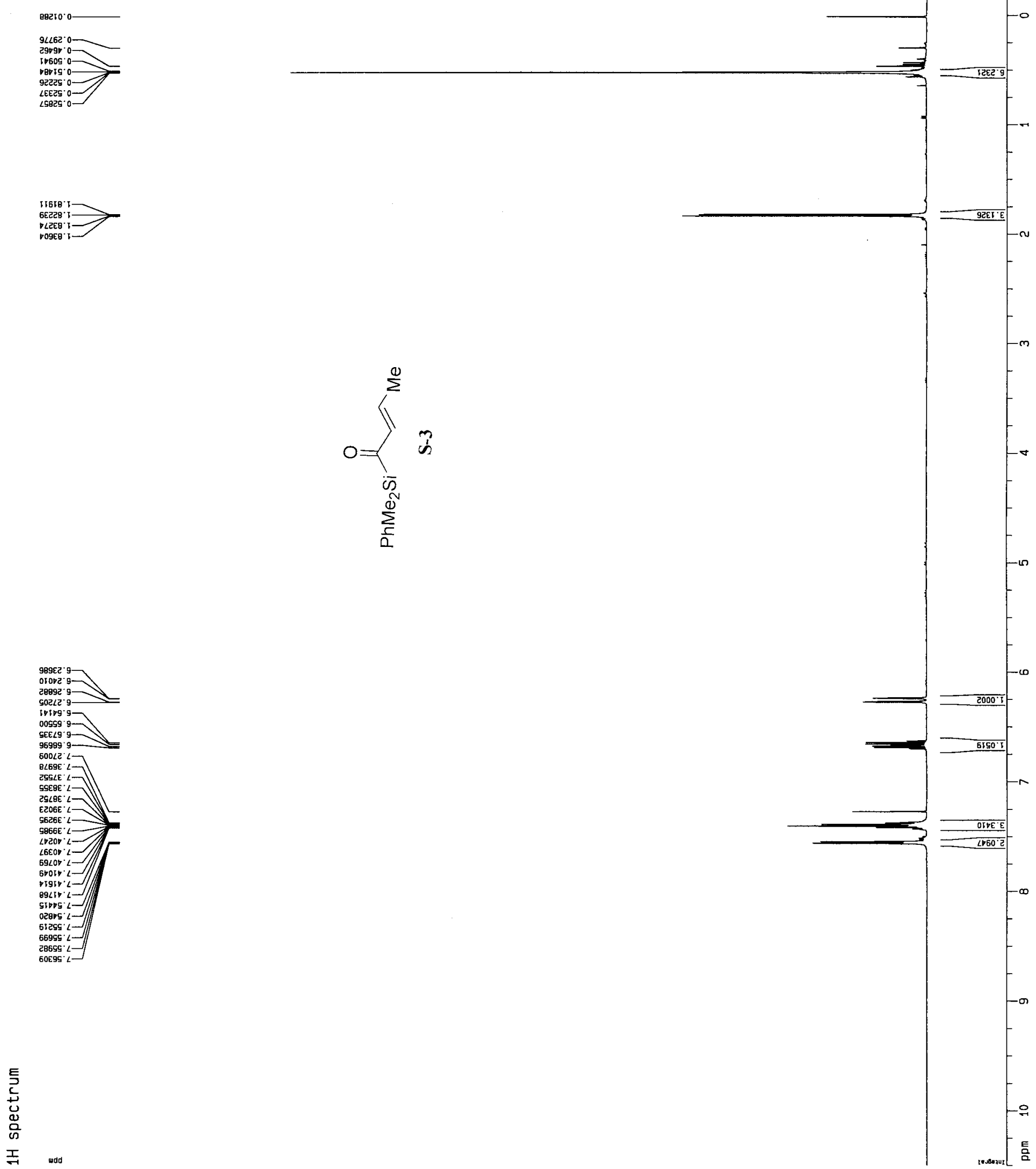


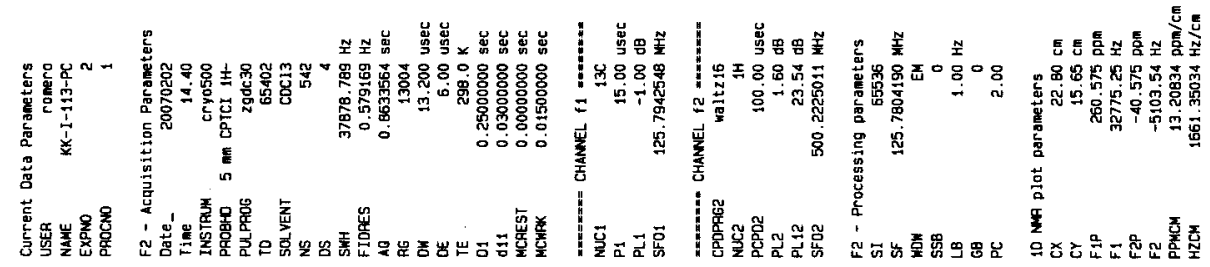

9v' $\varepsilon-$

$E C^{\prime}$ ir

0. $92 \mathrm{Z}$

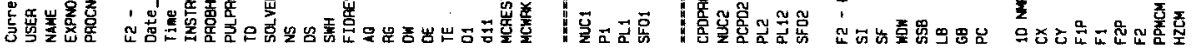

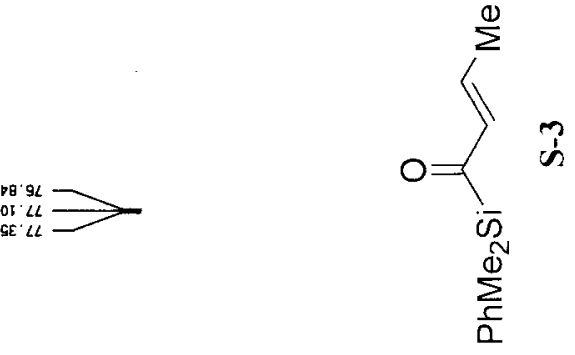

$25 \cdot 8217$

$62 \cdot 6512$

$06.621 \longrightarrow$

I0. $\mathrm{BEI}$

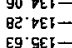

$\mathrm{Eg} \cdot \mathrm{SEl}$
$\mathrm{V} \cdot \mathrm{gEl}$

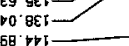

3

$\bar{s}$

돈

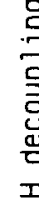

营

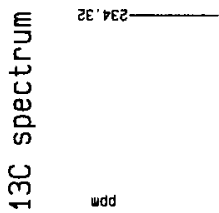



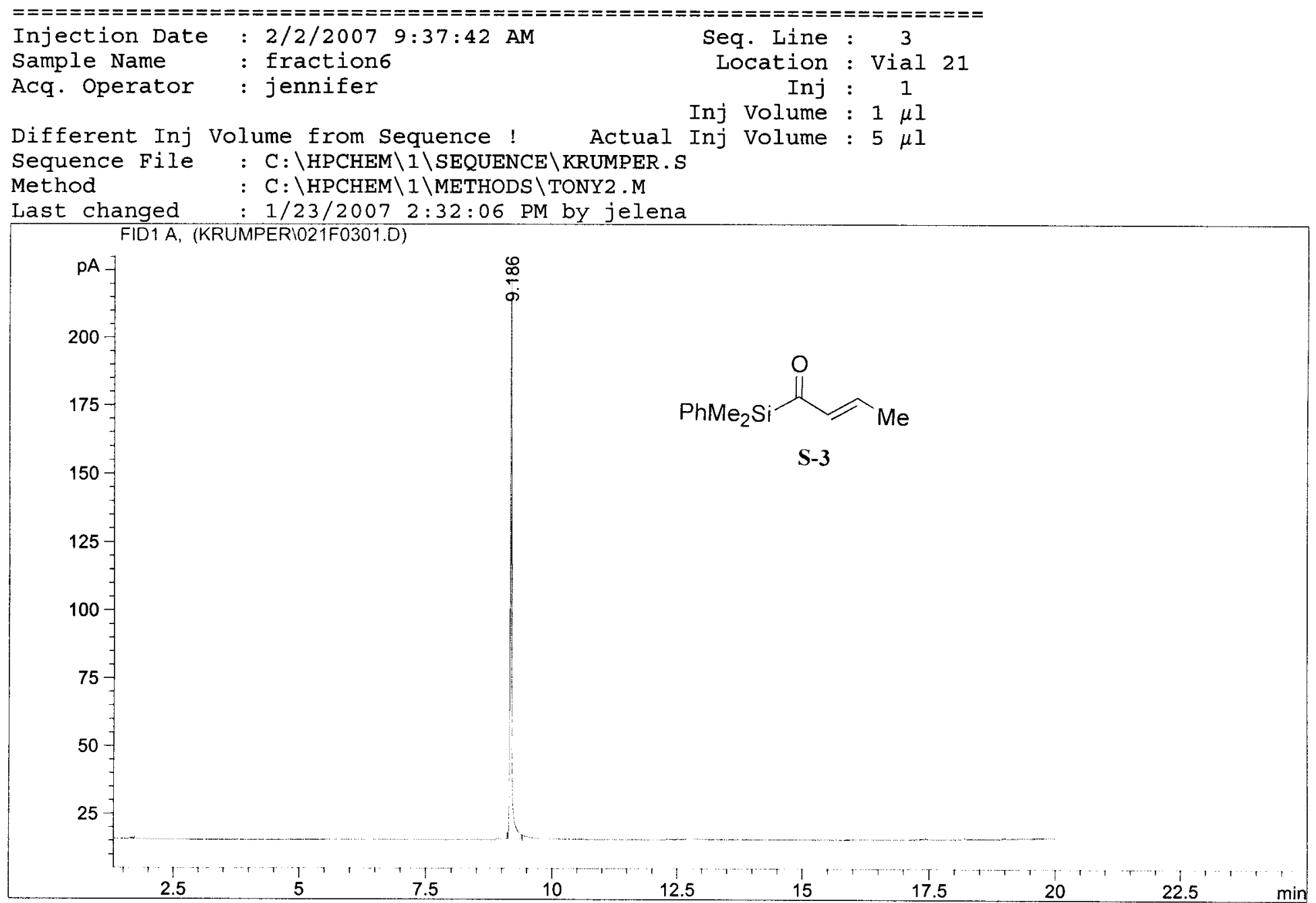

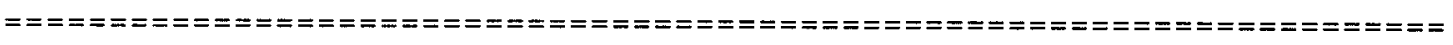

Area Percent Report

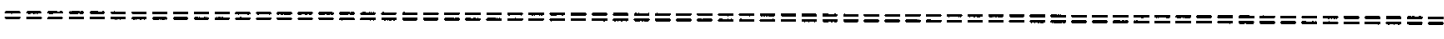

Sorted By

Multiplier

Signal

Dilution

1.0000

1.0000

Signal 1: FID1 A,

Peak RetTime Type width Area Height Area

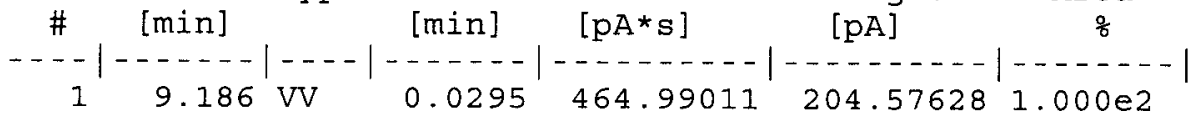

Totals : $\quad 464.99011204 .57628$

Results obtained with enhanced integrator!

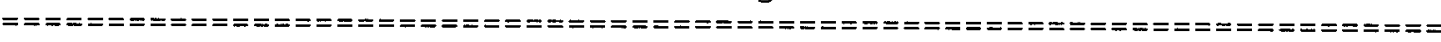

*** End of Report *** 


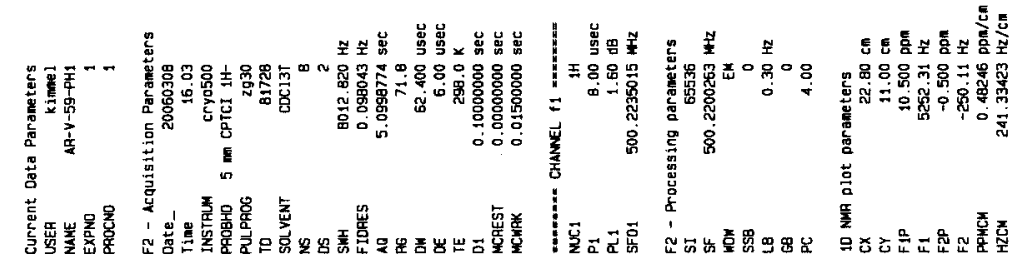

2RLCE: 0

zsas! :

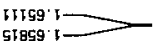

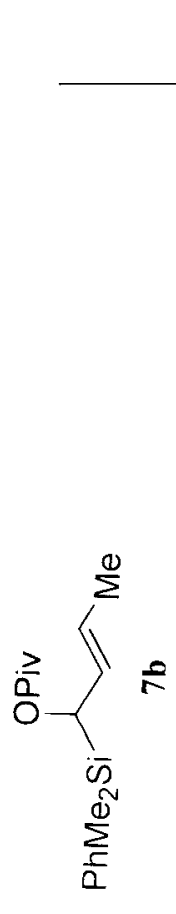

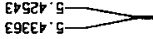

E6692: $2-$

IOBSE: $L \longrightarrow$

g995: $L$

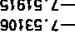

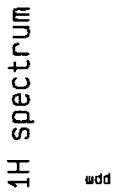

$$
\text { ำ }
$$

$\sqrt{2}$

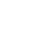




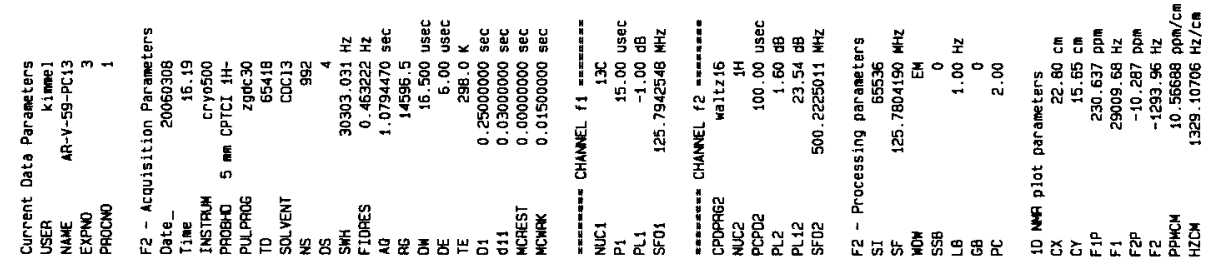

$\underset{\substack{05 \\ 91 \mathrm{~s}-}}{{ }_{\mathrm{s}}}$

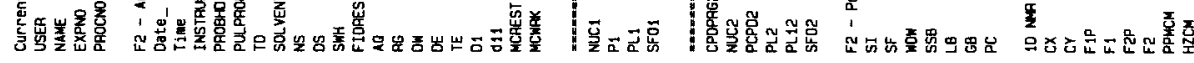

$$
00 \cdot 85
$$

$\varepsilon \varepsilon \cdot \measuredangle 2$

$00^{\circ} 6 \varepsilon$

$28 \cdot 9 L$

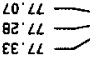

ER:

$9<\cdot 22] \longrightarrow$

6I DEI
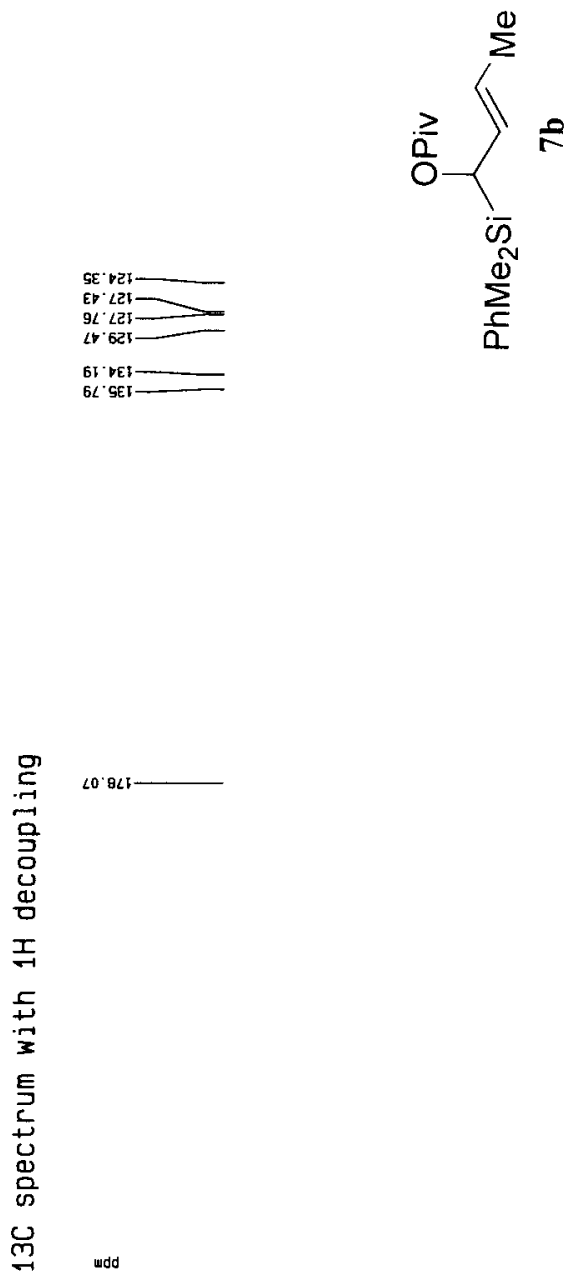

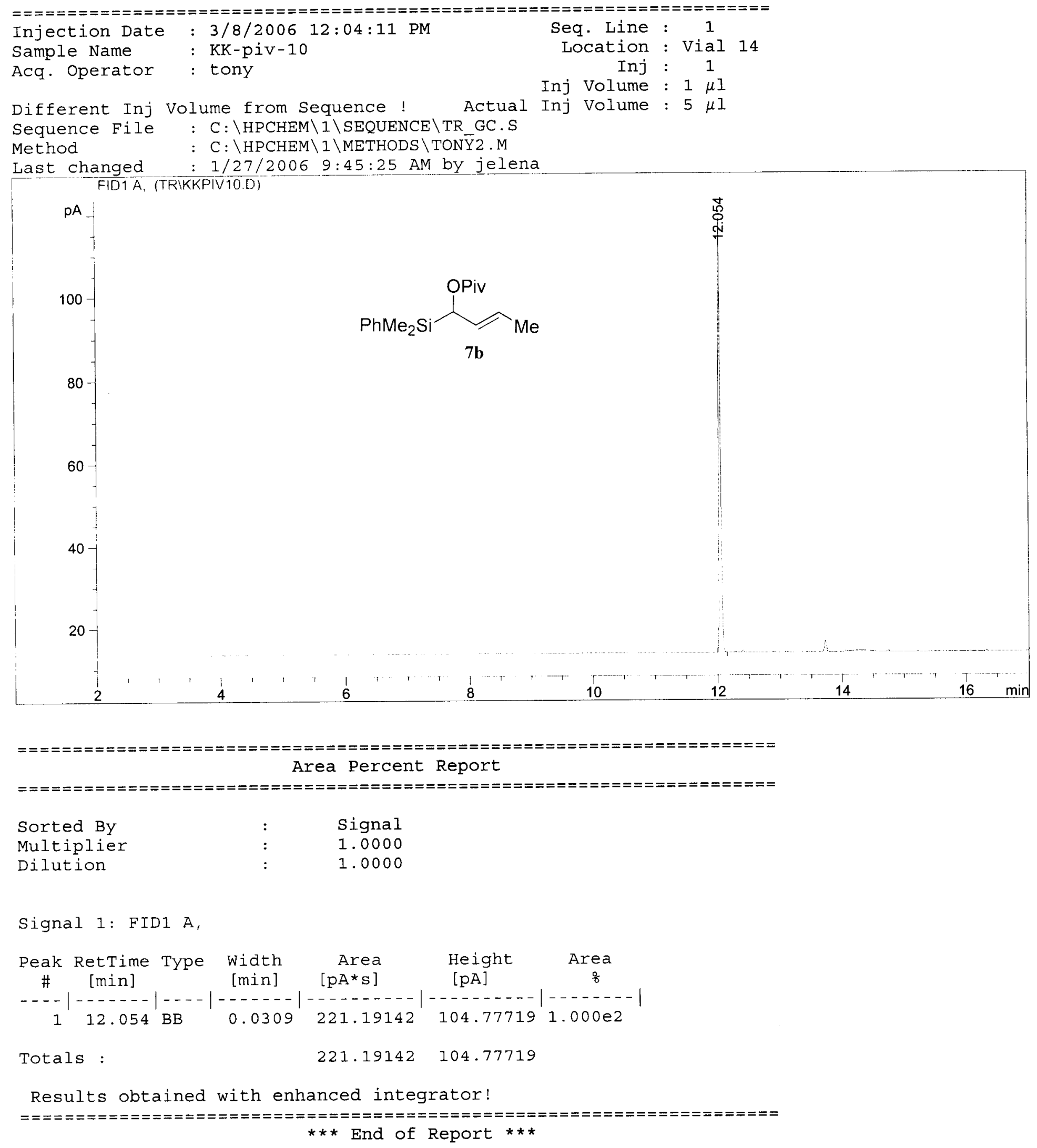


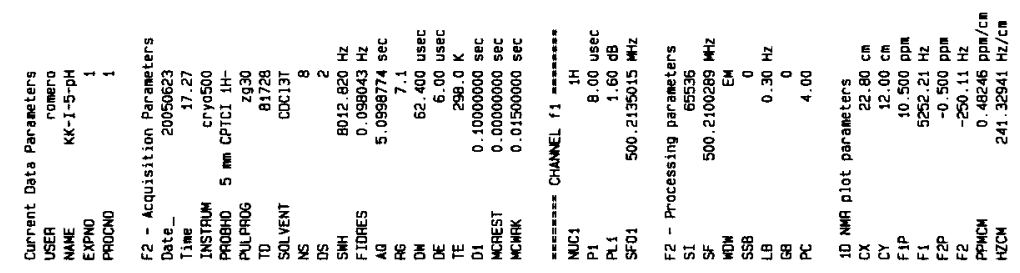

$p 9 \cdot 6 r$
$s !<r$

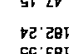

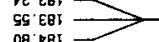

E8: 996

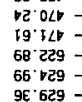

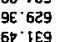

86: 9912

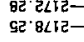

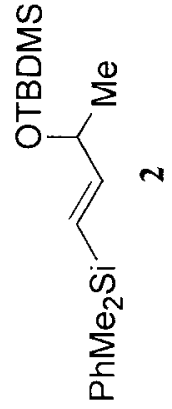

${ }^{80} \cdot \mathcal{E} 6262-$

or 2662

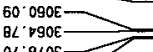

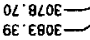

80 .

Eg.

$\varepsilon 2 \cdot 069 \varepsilon-1$

$12 \cdot 669 \mathrm{E}$

I. $24 L \varepsilon$

जG. $L L L E-$

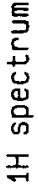

. 


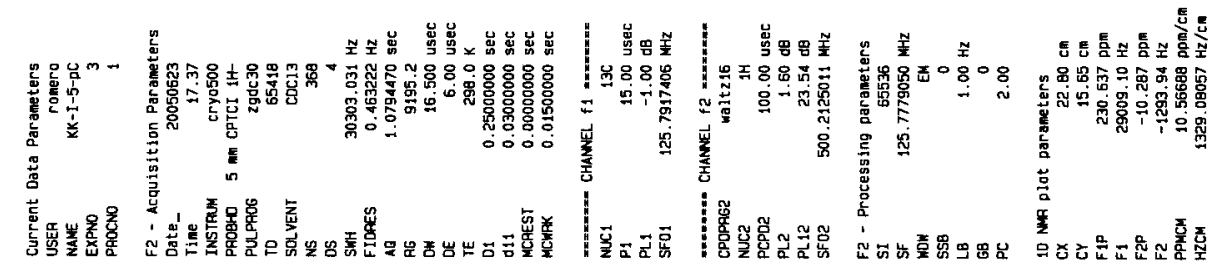

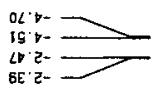

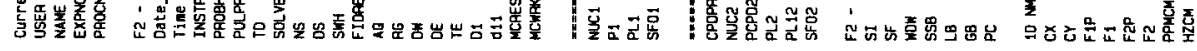

10.02
00.92

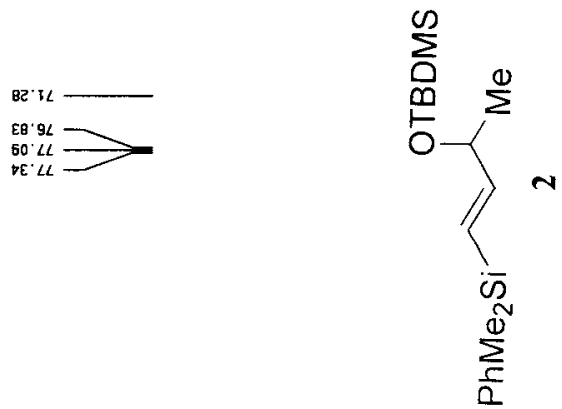

99' $v$ (2)

B. 6.825

ZE' $E E$ :

$00.6 \mathrm{E}$

E' 2 ' 

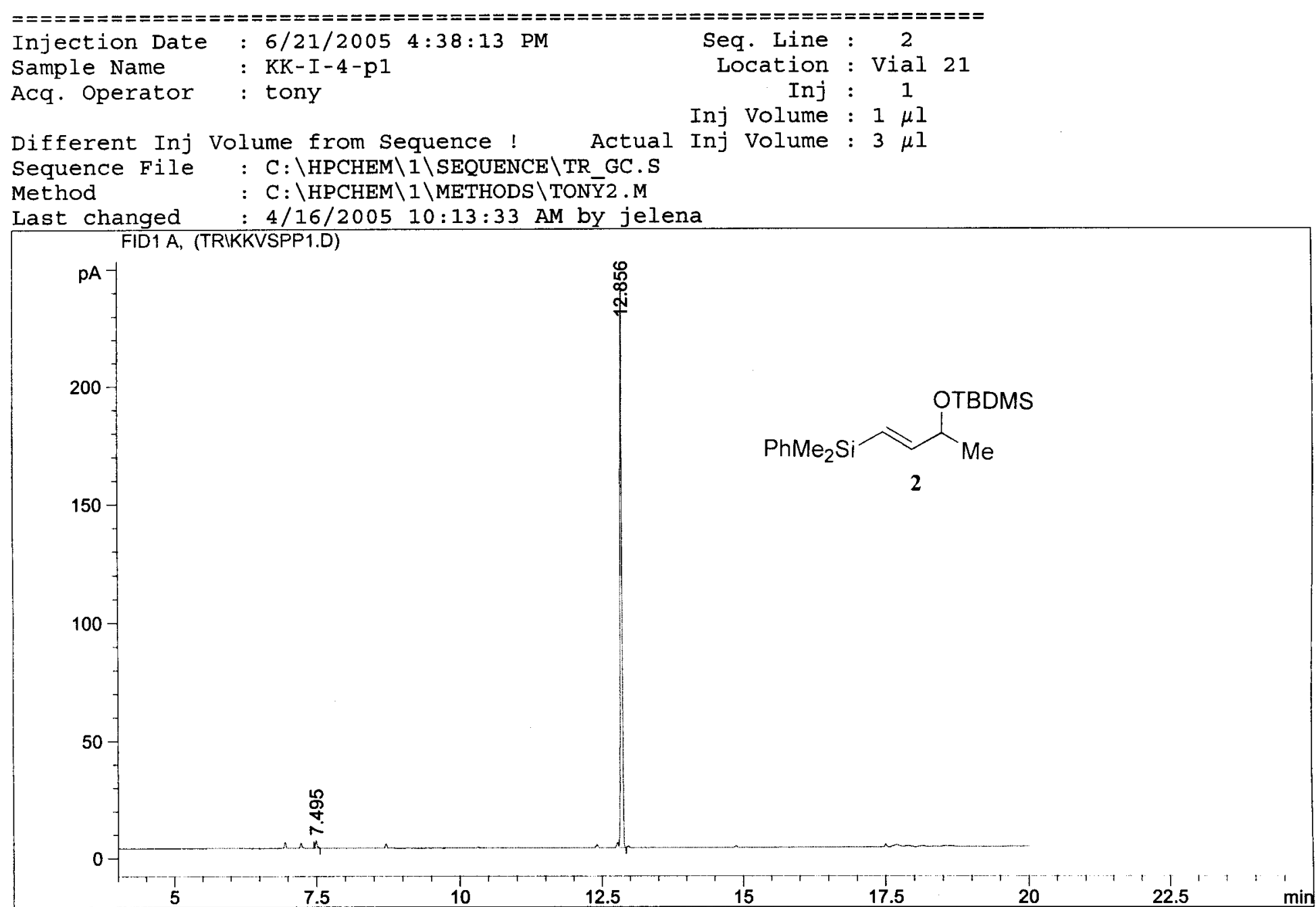

Area Percent Report

$\begin{array}{lll}\text { Sorted By } & : & \text { Signal } \\ \text { Multiplier } & : & 1.0000 \\ \text { Dilution } & : & 1.0000\end{array}$

Signal 1: FID1 A,

\begin{tabular}{|c|c|c|c|c|c|c|}
\hline $\begin{array}{c}\text { Peak } \\
\quad \#\end{array}$ & $\begin{array}{c}\text { RetTime } \\
\text { [min] }\end{array}$ & Type & $\begin{array}{c}\text { Width } \\
\text { [min] }\end{array}$ & $\begin{array}{r}\text { Area } \\
{\left[\mathrm{pA}^{*} \mathrm{~s}\right]}\end{array}$ & $\begin{array}{l}\text { Height } \\
{[\mathrm{pA}]}\end{array}$ & $\begin{array}{c}\text { Area } \\
\frac{q}{6}\end{array}$ \\
\hline 1 & & & & 615000 & & \\
\hline $\begin{array}{l}1 \\
2\end{array}$ & 7.495 & BB & 0.0261 & 6.45280 & 3.18348 & 1.26378 \\
\hline 2 & 12.856 & VB & 0.0263 & 504.14166 & 237.26180 & 98.13622 \\
\hline \multicolumn{4}{|c|}{ Totals : } & 510.59446 & 240.44527 & \\
\hline
\end{tabular}

Results obtained with enhanced integrator! 


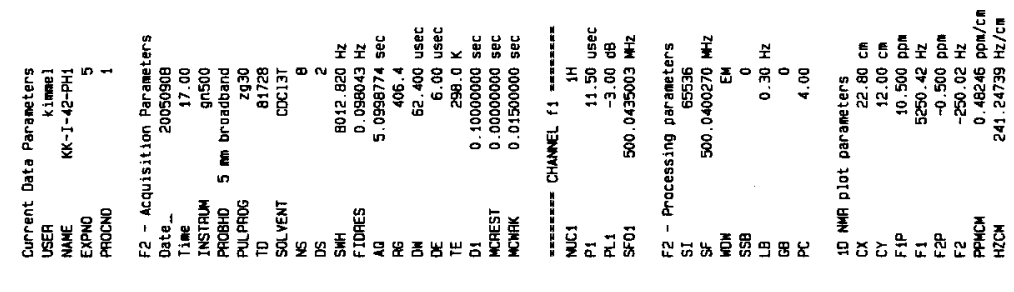

68:

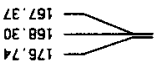

${ }_{8 \mathrm{r}}^{80 \cdot \mathrm{r} e \mathrm{r}} \mathrm{\gamma}$

$60.05 \%$

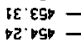

$E 2 \cdot 0 L_{L}$
gr $: 4 L_{L}$

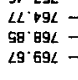

$\angle 9.69 \%$

\section{垔}

(1)

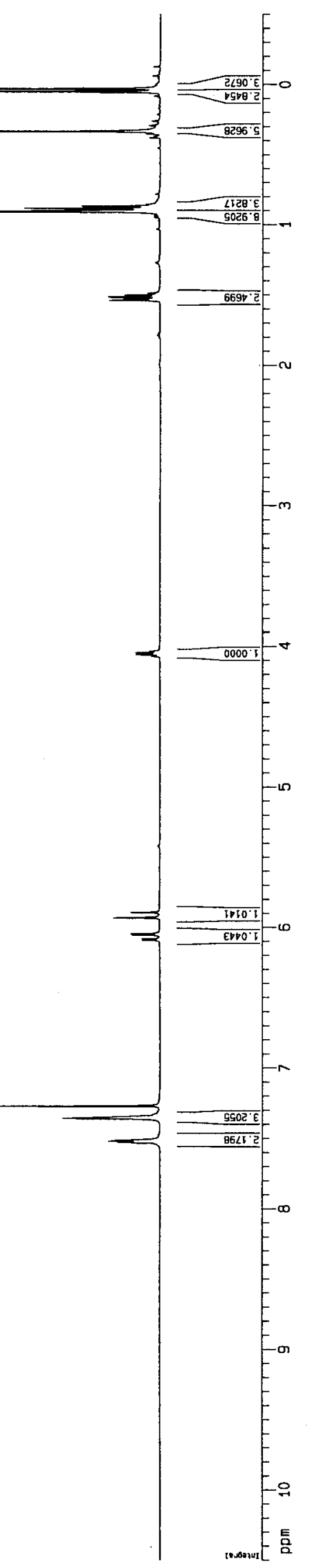




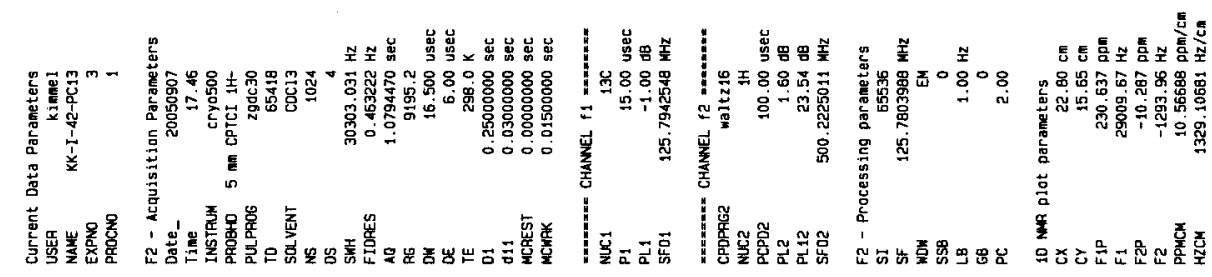

敫,

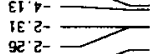

$250=$

$56^{*} 6$

95' 81

$\varepsilon \mathrm{El} \cdot \mathrm{gz}$

DQ: OE

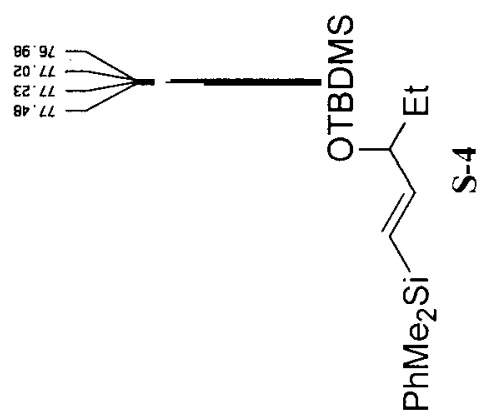

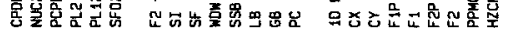

\section{然}



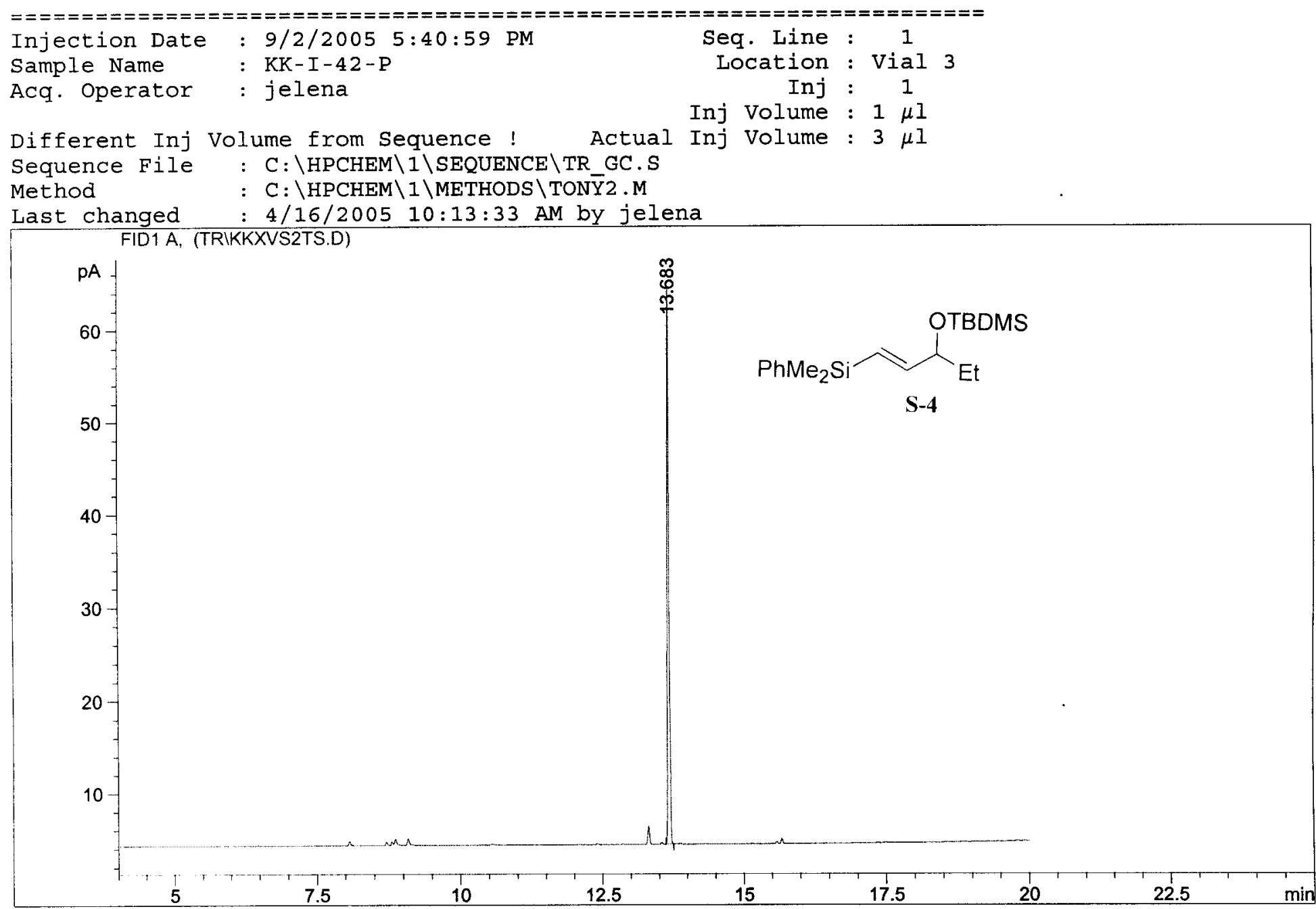

\begin{tabular}{|c|c|c|}
\hline Sorted By & : & Signal \\
\hline Multiplier & : & 1.0000 \\
\hline
\end{tabular}

Signal 1: FID1 A,

Peak RetTime Type Width Area Height Area

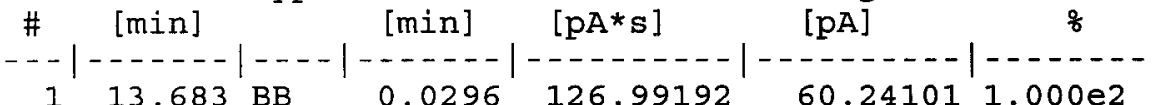

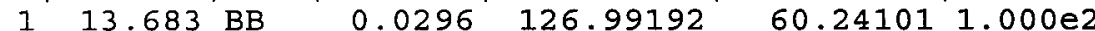

Totals : $\quad 126.99192 \quad 60.24101$

Results obtained with enhanced integrator! 


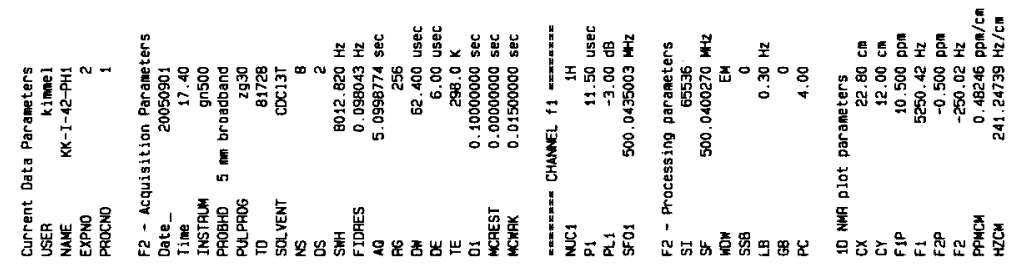

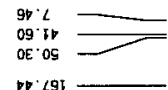

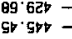

$62 \cdot 25 t=2$

18.

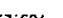

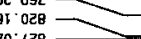

69. 228

ce.

so. $8912=$

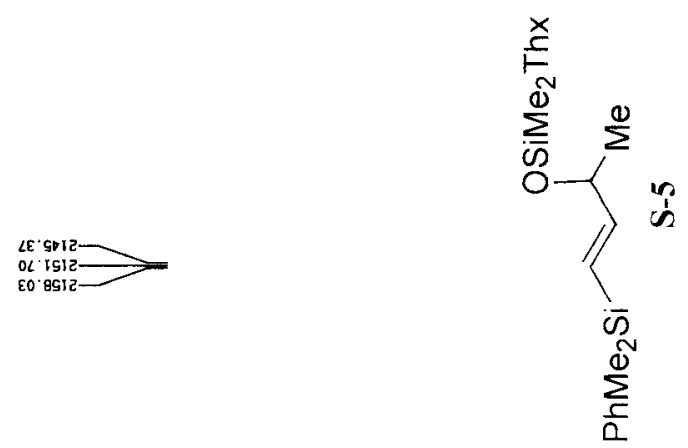

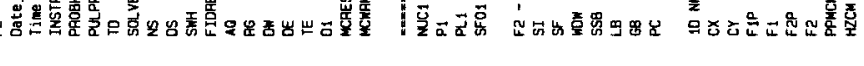

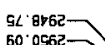

LE. $2962-1$

16. 8962

oc. $\angle \mathrm{CrOE}-$

टE $2900 \mathrm{E}$

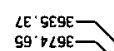

G9' $\angle L S E-$

$68^{\circ} \mathrm{BLE}-\mathrm{L}$

25. $\mathrm{G} L \mathrm{E}$

: $2 \cdot 69 \angle E-$

of E9LE-

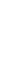


Supporting Information: Kim, Kimmel, Romero, Smitrovich, and Woerpel

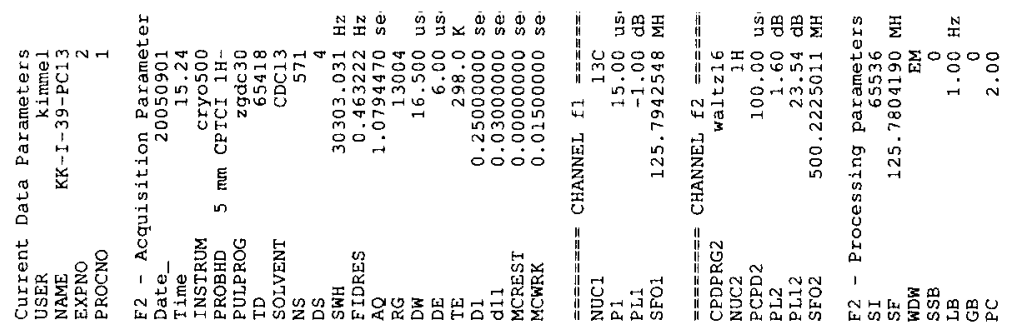

$089 \cdot 2-2$

$226 \cdot 2-2=$

26I.

$\operatorname{s\varepsilon 9\cdot 8\tau =}$

$\mathrm{IZL} \cdot \mathrm{ZZ} \longrightarrow$

I 2 I $5.52=$

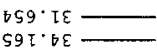

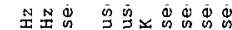

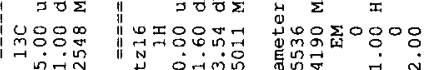

$690^{\circ} \angle L=$

$\angle 8 \mathrm{~S}^{\circ} 6 \mathrm{ZI}$

${ }_{\text {IS } L \cdot}^{\circ} \cdot \operatorname{LZT}=$

武高

乙68 $\varepsilon \varepsilon \tau$

$\llcorner\varepsilon 0 \cdot 6 \varepsilon \mathrm{I}$

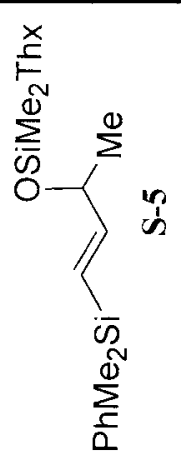

$9 \varepsilon \varsigma^{\prime}$ วร

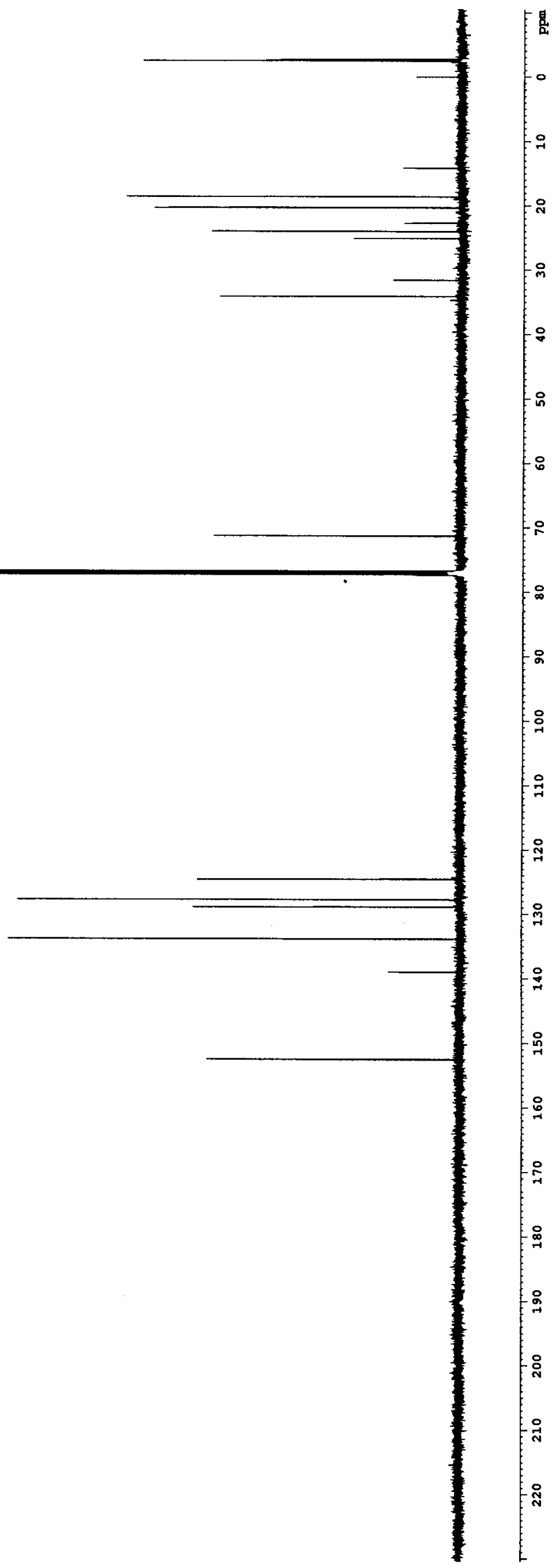




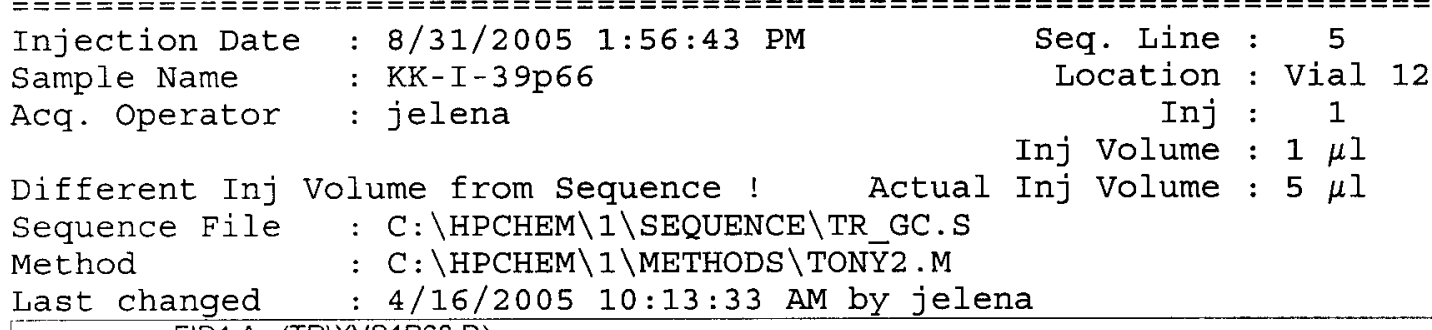

Inj Volume : $1 \mu \mathrm{I}$

Different Inj Volume from Sequence !

Sequence File : C: $\backslash$ HPCHEM $\backslash 1 \backslash S E Q U E N C E \backslash T R \_G C . S$

Method : C: $\backslash$ HPCHEM $\backslash 1 \backslash$ METHODS $\backslash T O N \bar{Y} 2 . M$

Last changed : 4/16/2005 10:13:33 AM by jelena

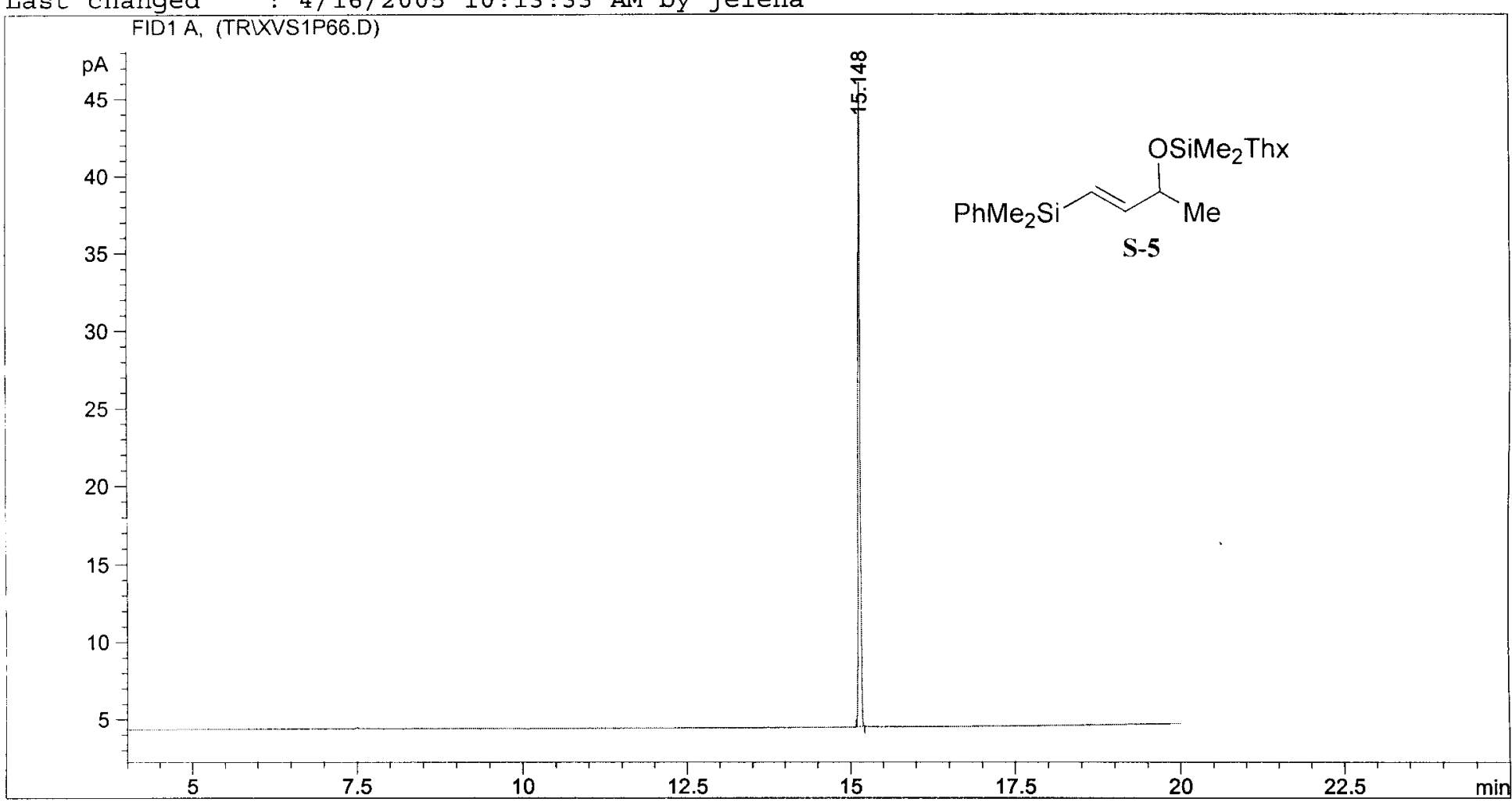

\begin{tabular}{|c|c|c|}
\hline Sorted By & : & Signal \\
\hline Multiplier & $:$ & 1.0000 \\
\hline Dilution & : & 1.0000 \\
\hline
\end{tabular}

Signal 1: FID1 A,

Peak RetTime Type width Area Height Area

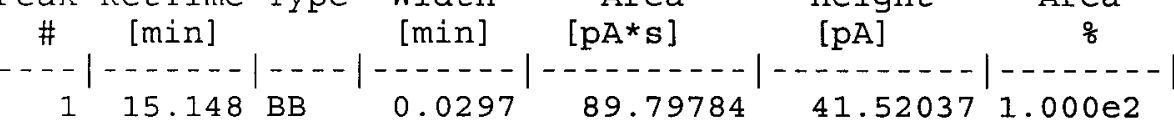

Totals :

$89.79784 \quad 41.52037$

Results obtained with enhanced integrator!

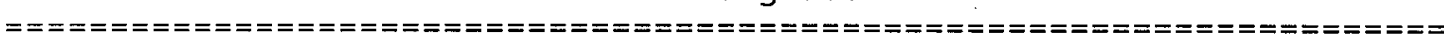

*** End of Report *** 

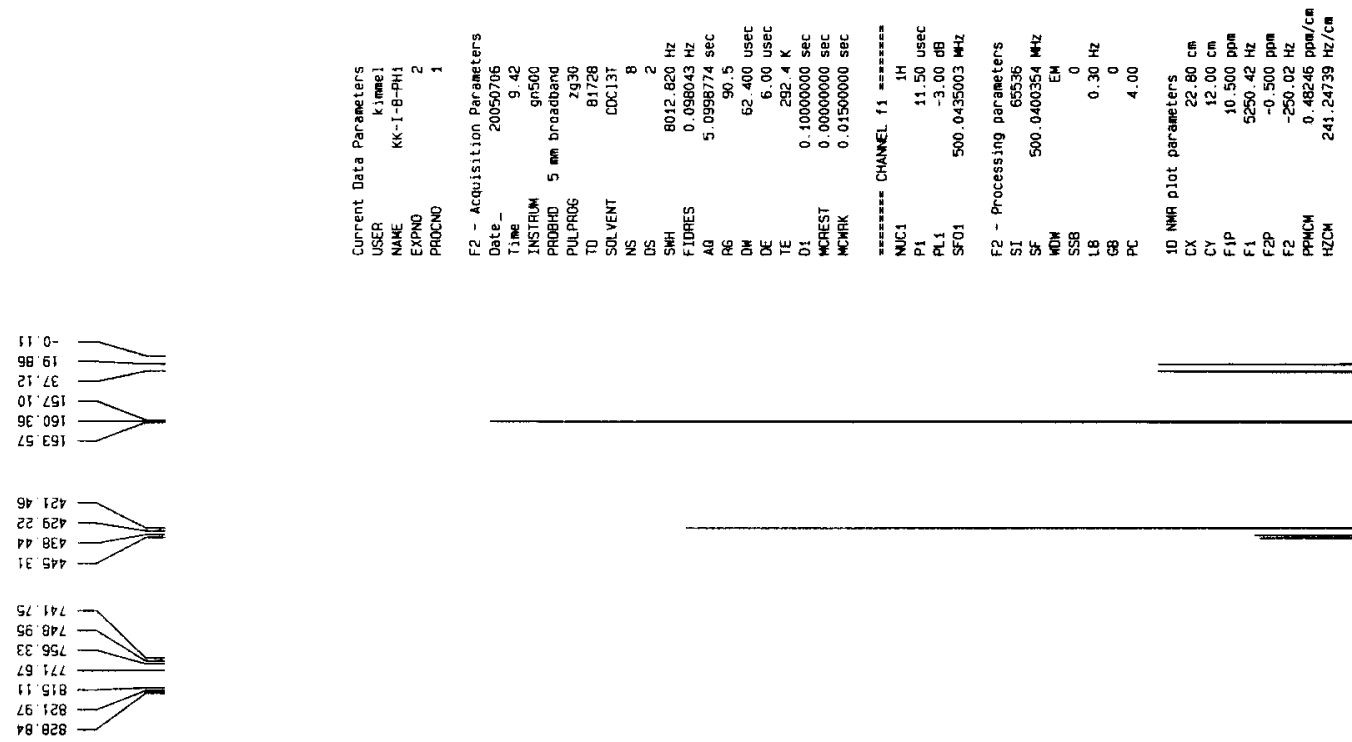

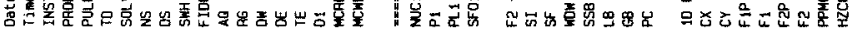
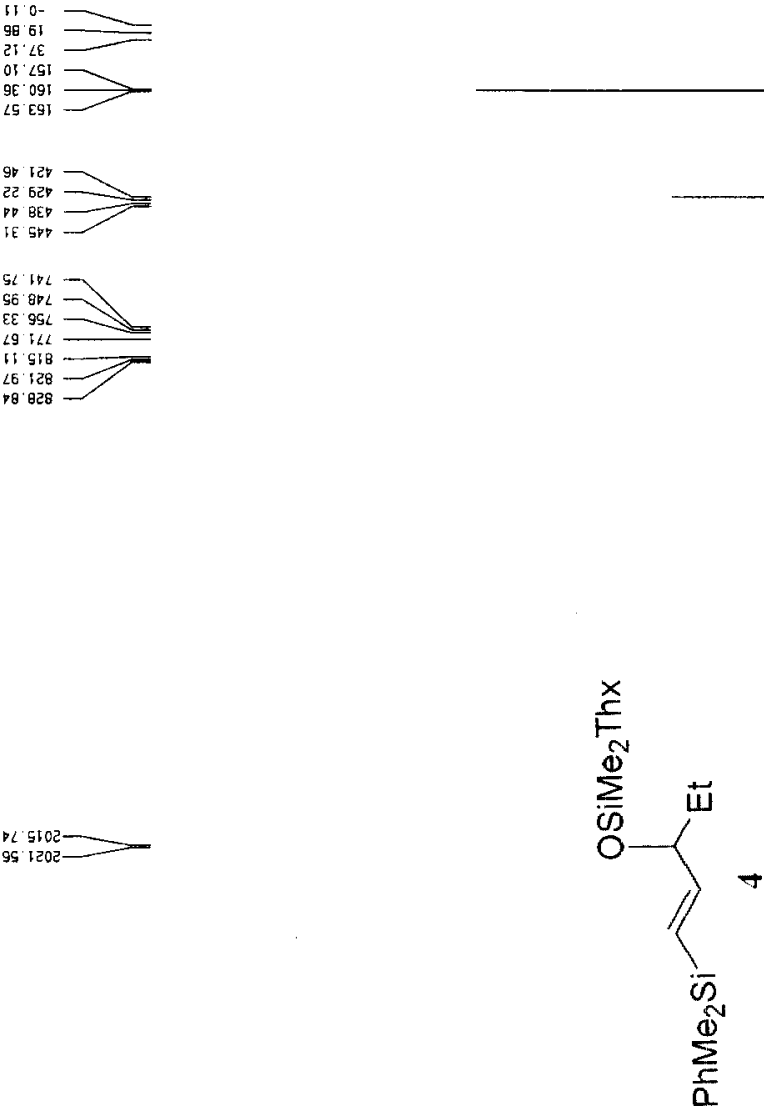

aE $\mathrm{pesc}$

$017562+252$

86.0106

LS $9100=7$

$99.6206-$

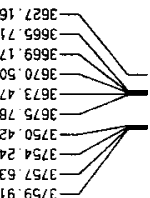



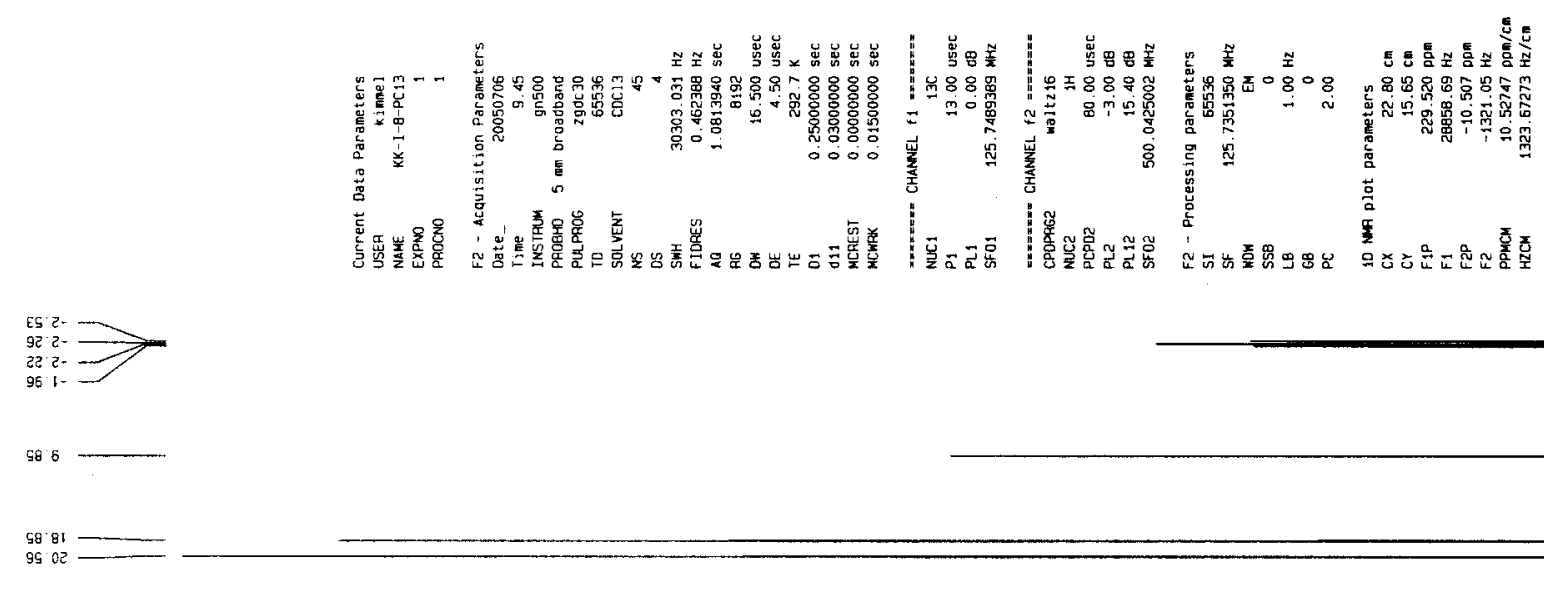

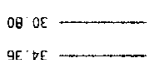

$9 E^{\prime} \not E$

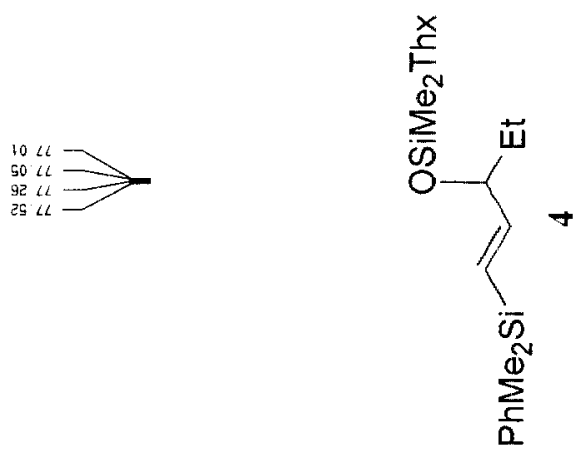

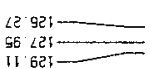

$80 \cdot 7 E$ I-
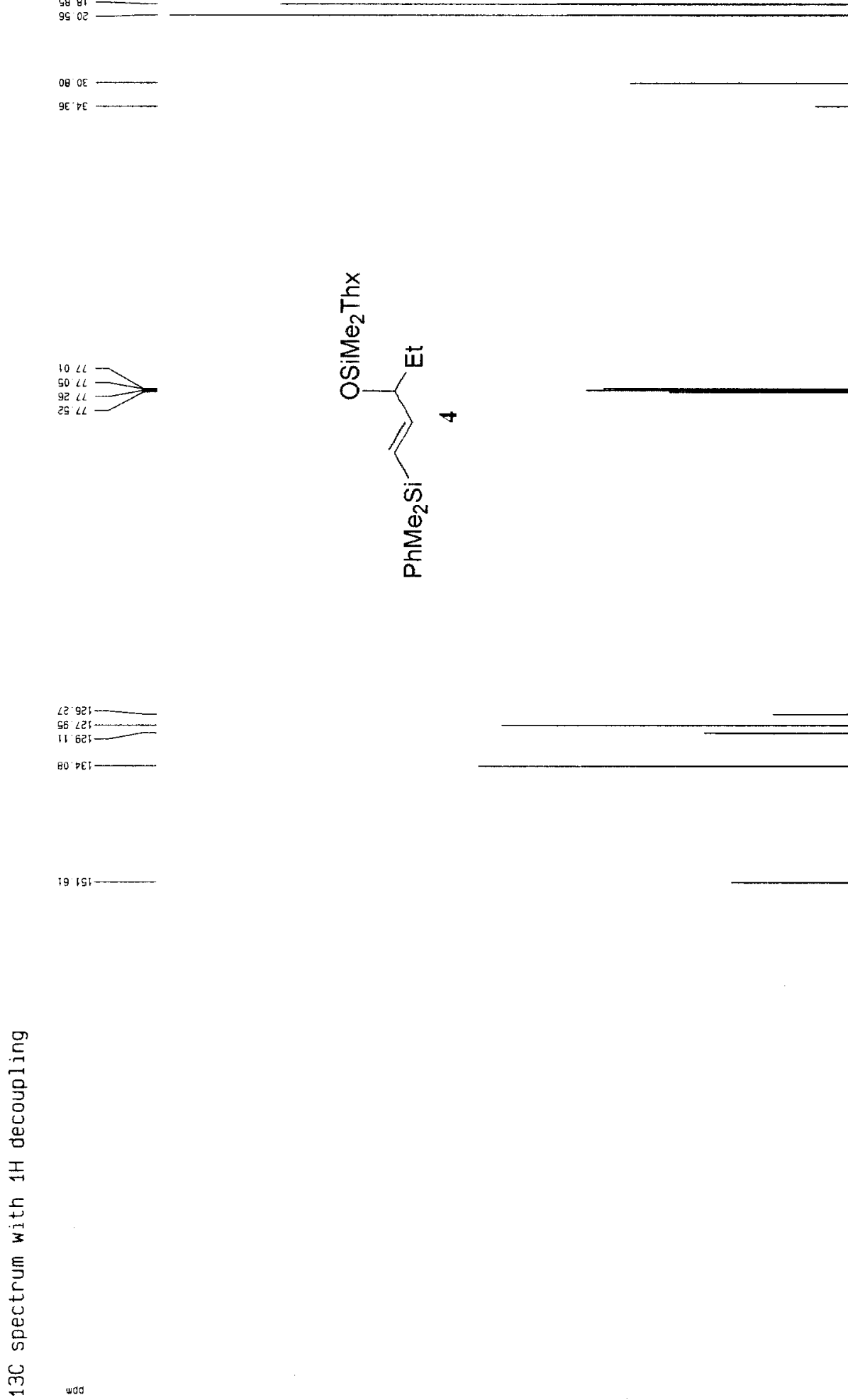


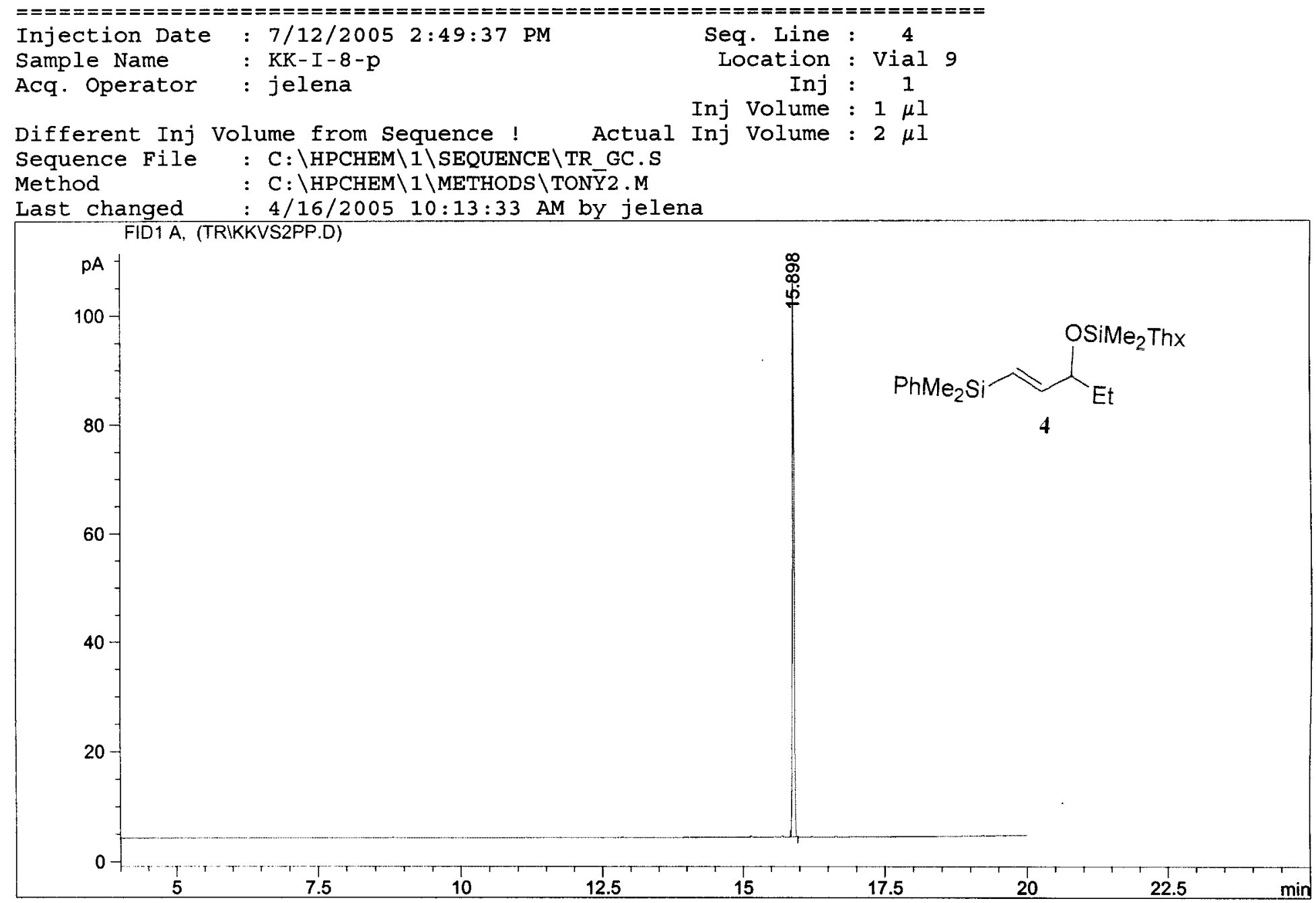

\begin{tabular}{|c|c|c|}
\hline Sorted By & : & Signal \\
\hline Multiplier & : & 1.0000 \\
\hline Dilution & : & 1.0000 \\
\hline
\end{tabular}

Signal 1: FID1 A,

\begin{tabular}{|c|c|c|c|c|c|c|}
\hline $\begin{array}{c}\text { Peak } \\
\#\end{array}$ & $\begin{array}{l}\text { RetTime } \\
\text { [min] }\end{array}$ & Type & $\begin{array}{c}\text { Width } \\
\text { [min] }\end{array}$ & $\begin{array}{r}\text { Area } \\
{\left[\mathrm{pA}^{*} \mathrm{~s}\right]}\end{array}$ & $\begin{array}{l}\text { Height } \\
\text { [pA] }\end{array}$ & $\begin{array}{c}\text { Area } \\
\frac{8}{8}\end{array}$ \\
\hline 1 & 15.898 & $\mathrm{BB}$ & 0.0281 & 221.56384 & 101.87508 & $1.000 \mathrm{e} 2$ \\
\hline ota & $:$ : & & & 221.56384 & & \\
\hline
\end{tabular}

Results obtained with enhanced integrator!

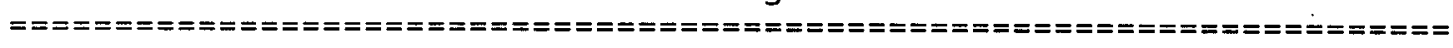

** End of Report *** 


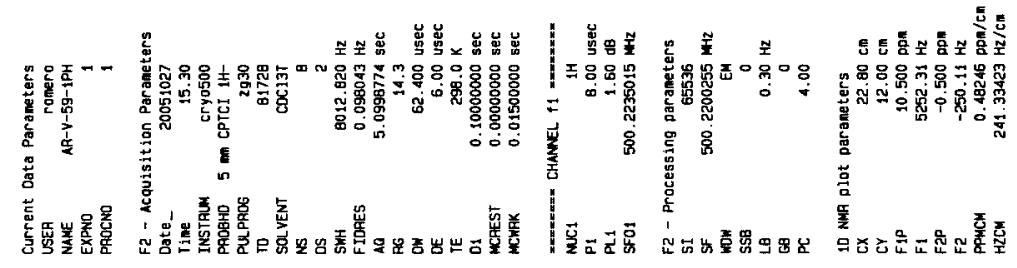

LZEgE - 0

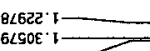

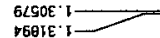

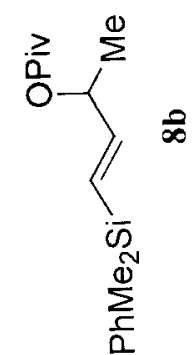

$\operatorname{ErgLE} \cdot \mathrm{s}$

$\infty$

$\bar{s}$

衣

$090 \angle 6 . \mathrm{g}$
$\square 2 \angle \angle 6 . \mathrm{G}$

E2ट250.9-

$88010.9-9$

$80060.9-$

$\angle 9 \angle 2,9$

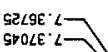

SPOLCE: 2 -

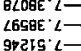

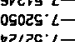

GELES:L二

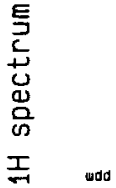




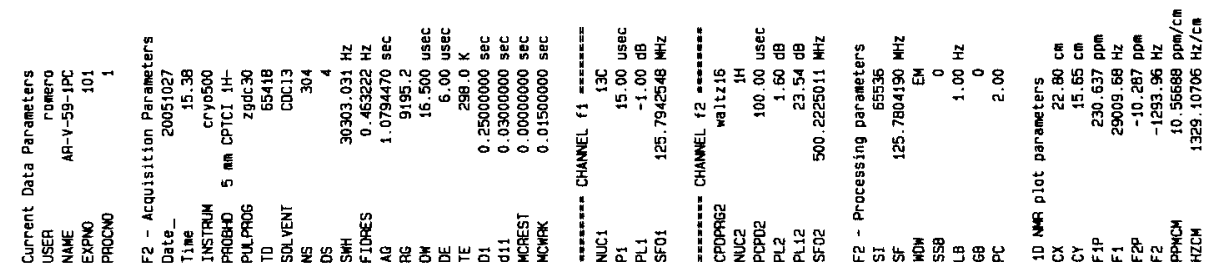

$\lg \cdot 2-=$

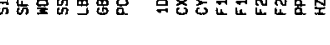

¿2 $\measuredangle 2$

${ }^{8 \theta^{\prime}} \theta \varepsilon$

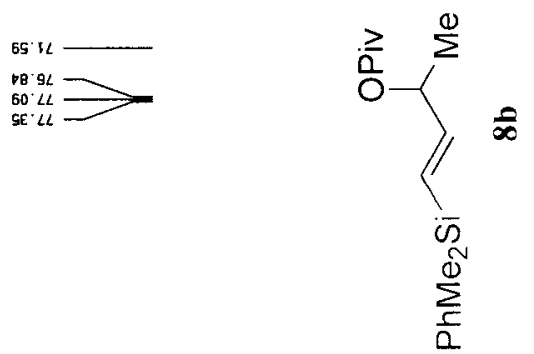

$29 \cdot 2 \mathrm{LI}-$

60. $62 \mathrm{~T}$

Б8' EE'

gr' $B E$ -

$90 \angle \mathrm{LT}$

$\frac{0}{\infty}$

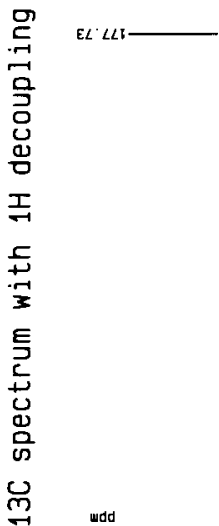


PIV protected vinylsilane
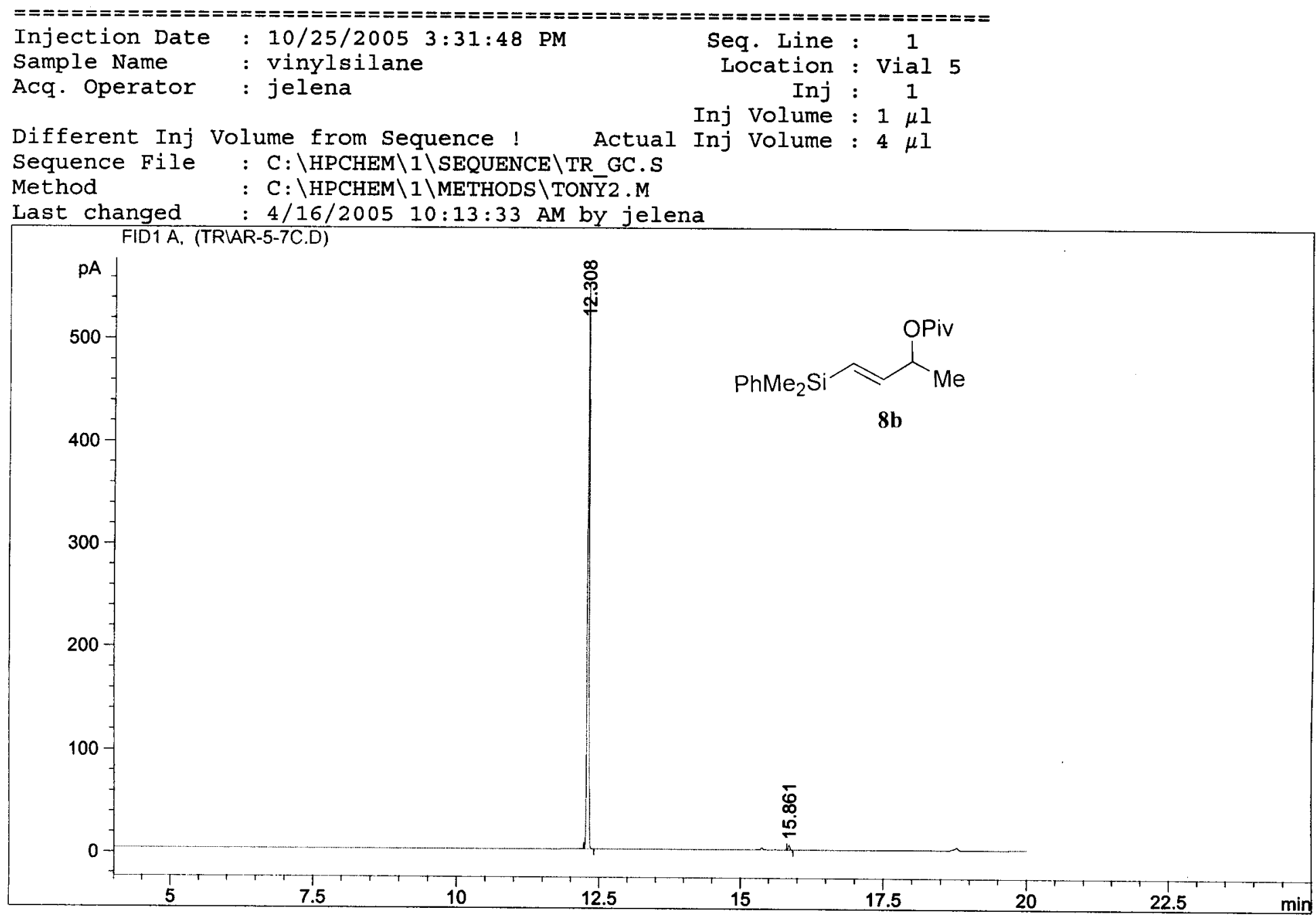

\section{Area Percent Report}

Sorted By

Multiplier

Dilution

\section{Signal}

1.0000

1.0000

Signal 1: FID1 A,

Peak RetTime Type Width Area Height Area

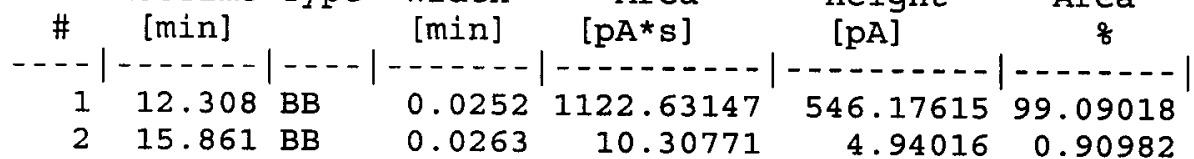

Totals :

$1132.93918 \quad 551.11631$

Results obtained with enhanced integrator! 


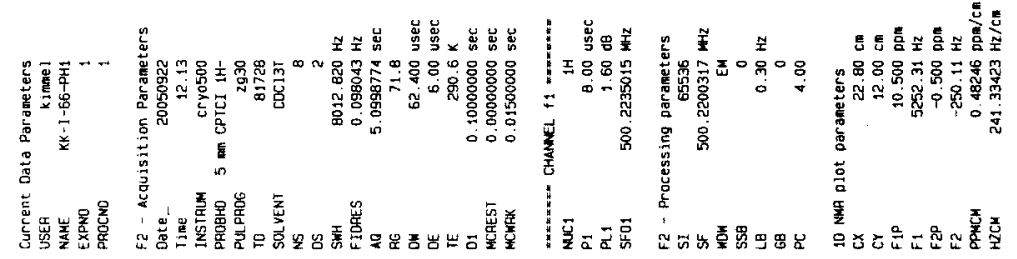

I:582: $1=$

EISGS :

IC9EE:

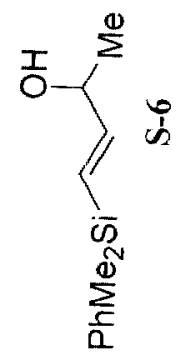

99656525

18206:9-2

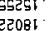

465819

$68652 \cdot 1$

¿tLSE:

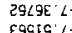

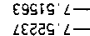

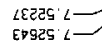

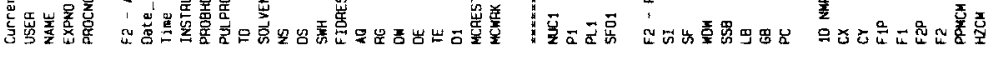




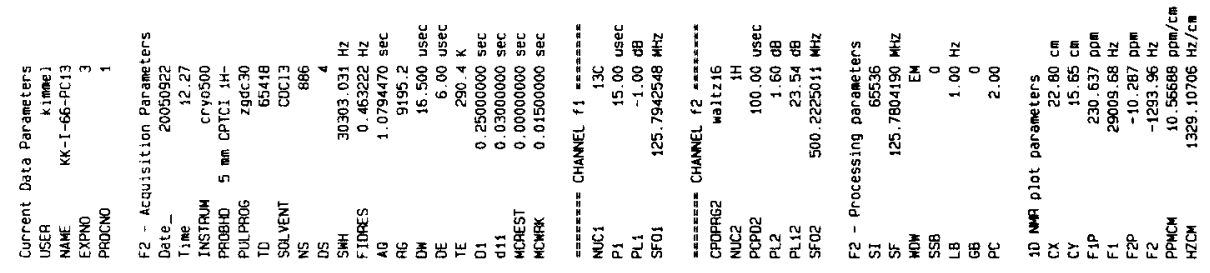

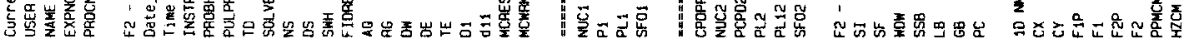

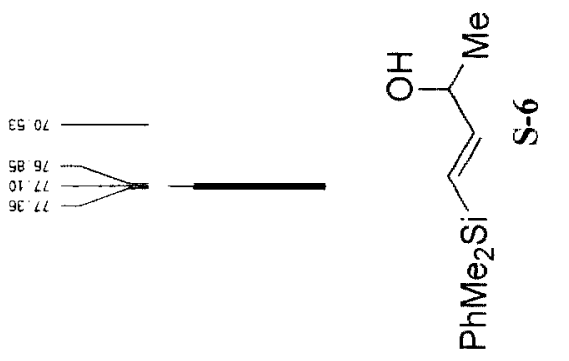

L6. $521=-$
$18.21=-$

$8 \mathrm{Q} \cdot \mathrm{EE}$

DS BEL

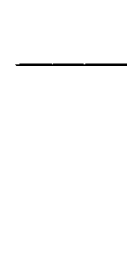



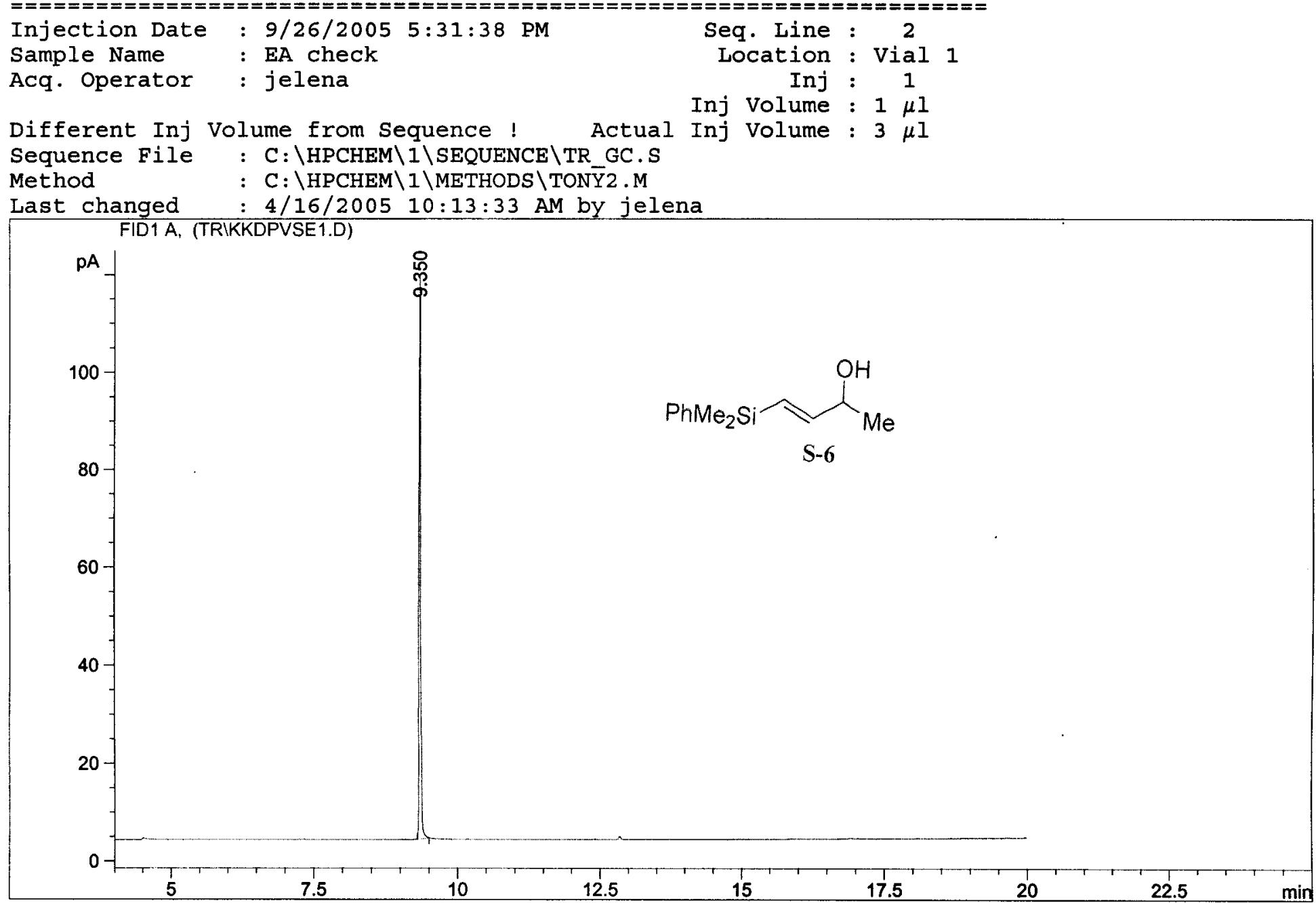

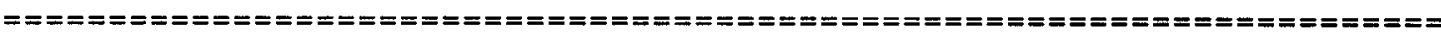

Area Percent Report

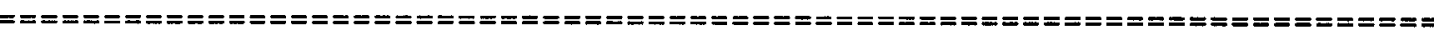

Sorted By

Multiplier

Dilution
Signal

1.0000

1.0000

Signal 1: FID1 A,

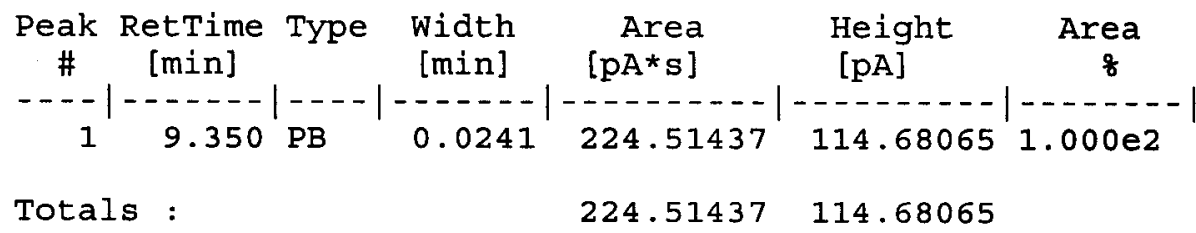

Results obtained with enhanced integrator! 


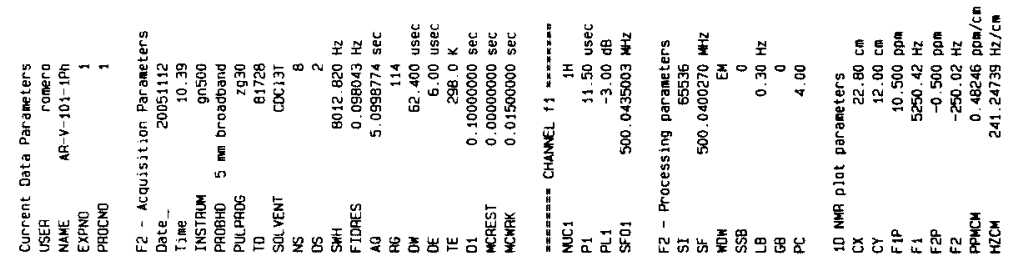

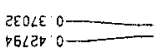

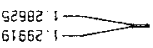

$\$ 9909:$

GETEE :

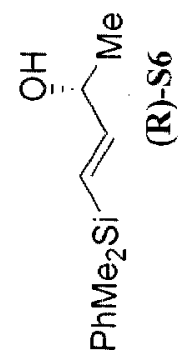

$80596 \cdot 2-$

$\operatorname{c\varepsilon c} \angle 6.5-1=$

$86900 \cdot 9$

-

$66149=$

(a)

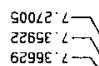

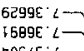

rosete : 2

is

2roce. 20.25

DLACS L-

ingers

$\frac{\infty}{2}$

$\bar{\omega}$

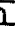


Supporting Information: Kim, Kimmel, Romero, Smitrovich, and Woerpel

$S-44$

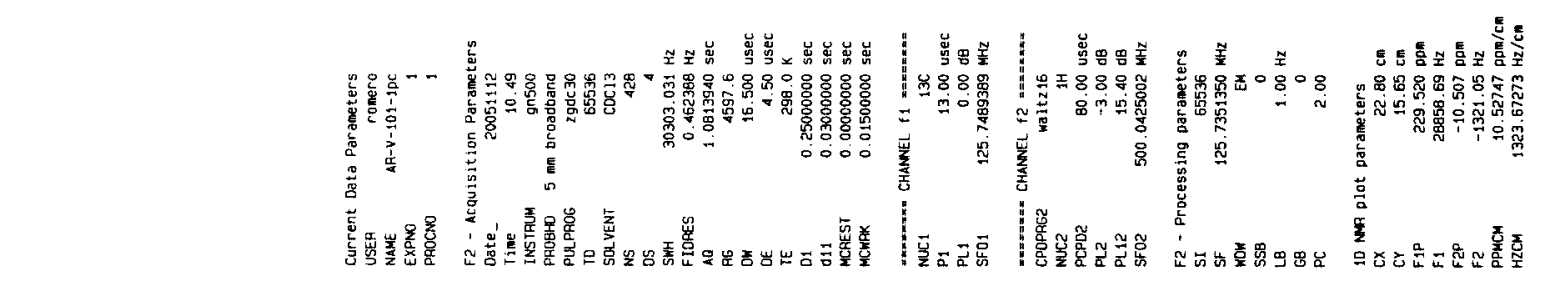

BE $2-\square$

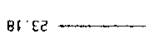

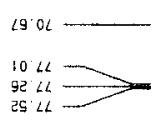

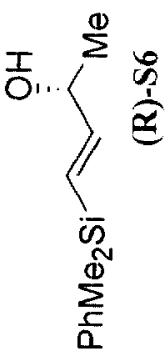

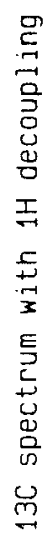

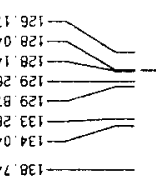

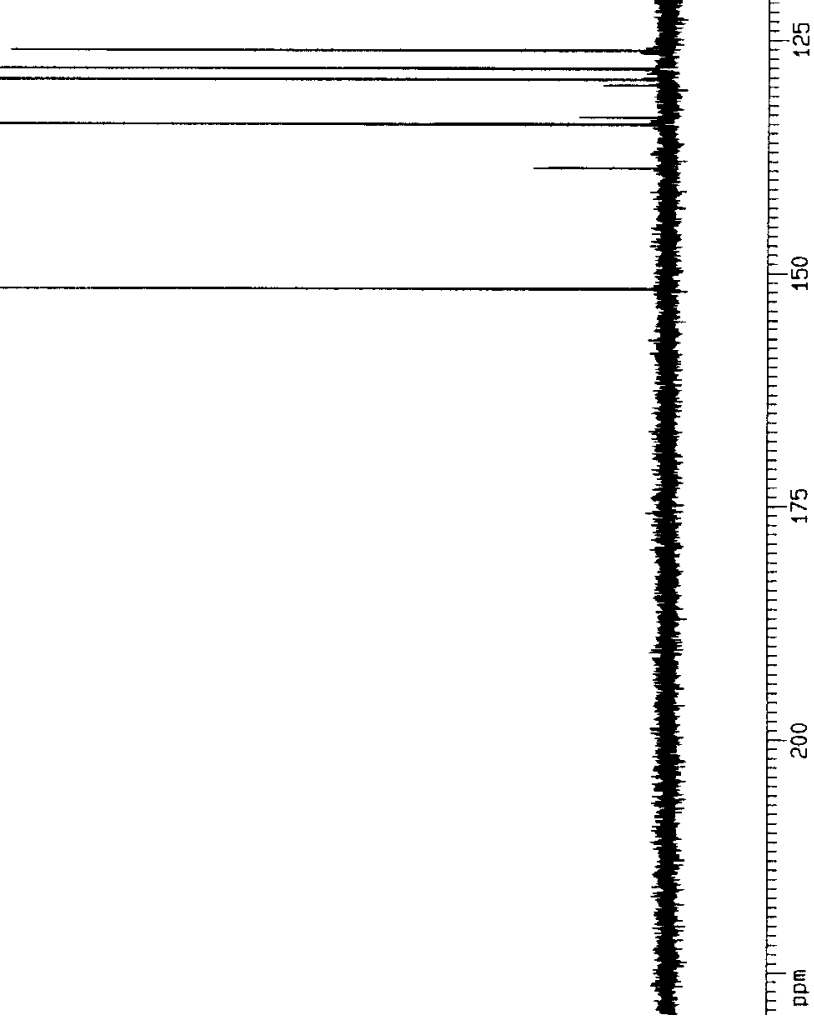

-

8

号

$9 \cdot 19 !$

E哀 


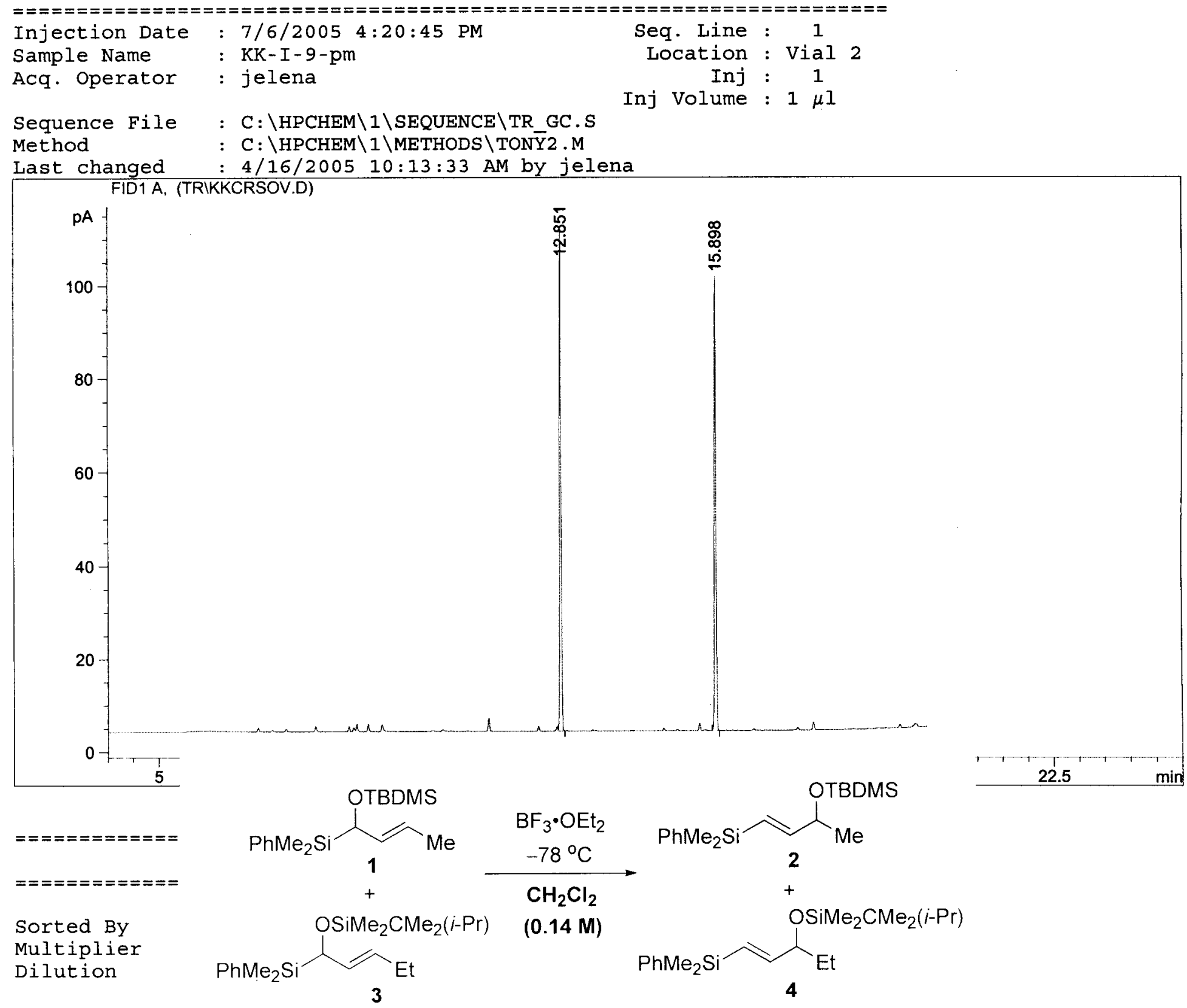

Signal 1: FID1 A,

\begin{tabular}{|c|c|c|c|c|c|c|}
\hline $\begin{array}{c}\text { Peak } \\
\#\end{array}$ & $\begin{array}{l}\text { RetTime } \\
\text { [min] }\end{array}$ & Type & $\begin{array}{l}\text { Width } \\
\text { [min] }\end{array}$ & $\begin{array}{r}\text { Area } \\
{\left[p A^{*} \mathrm{~s}\right]}\end{array}$ & $\begin{array}{l}\text { Height } \\
{[\mathrm{pA}]}\end{array}$ & $\begin{array}{c}\text { Area } \\
\frac{q}{b}\end{array}$ \\
\hline & & & & 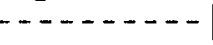 & & \\
\hline 1 & 1.425 & BB & 0.0112 & 4.63328 & 6.40631 & 1.04745 \\
\hline 2 & 2.590 & $\mathrm{~PB}$ & 0.0193 & 3.74237 & 3.00918 & 0.84604 \\
\hline 3 & 3.320 & BB & 0.0233 & 11.43873 & 7.58403 & 2.58597 \\
\hline 4 & 12.851 & VP & 0.0298 & 217.06615 & 107.47176 & 49.07250 \\
\hline 5 & 15.898 & BP & 0.0294 & 205.45709 & 97.31677 & 46.44803 \\
\hline & & & & 442.33762 & 78805 & \\
\hline
\end{tabular}

Results obtained with enhanced integrator!

*** End of Report *** 

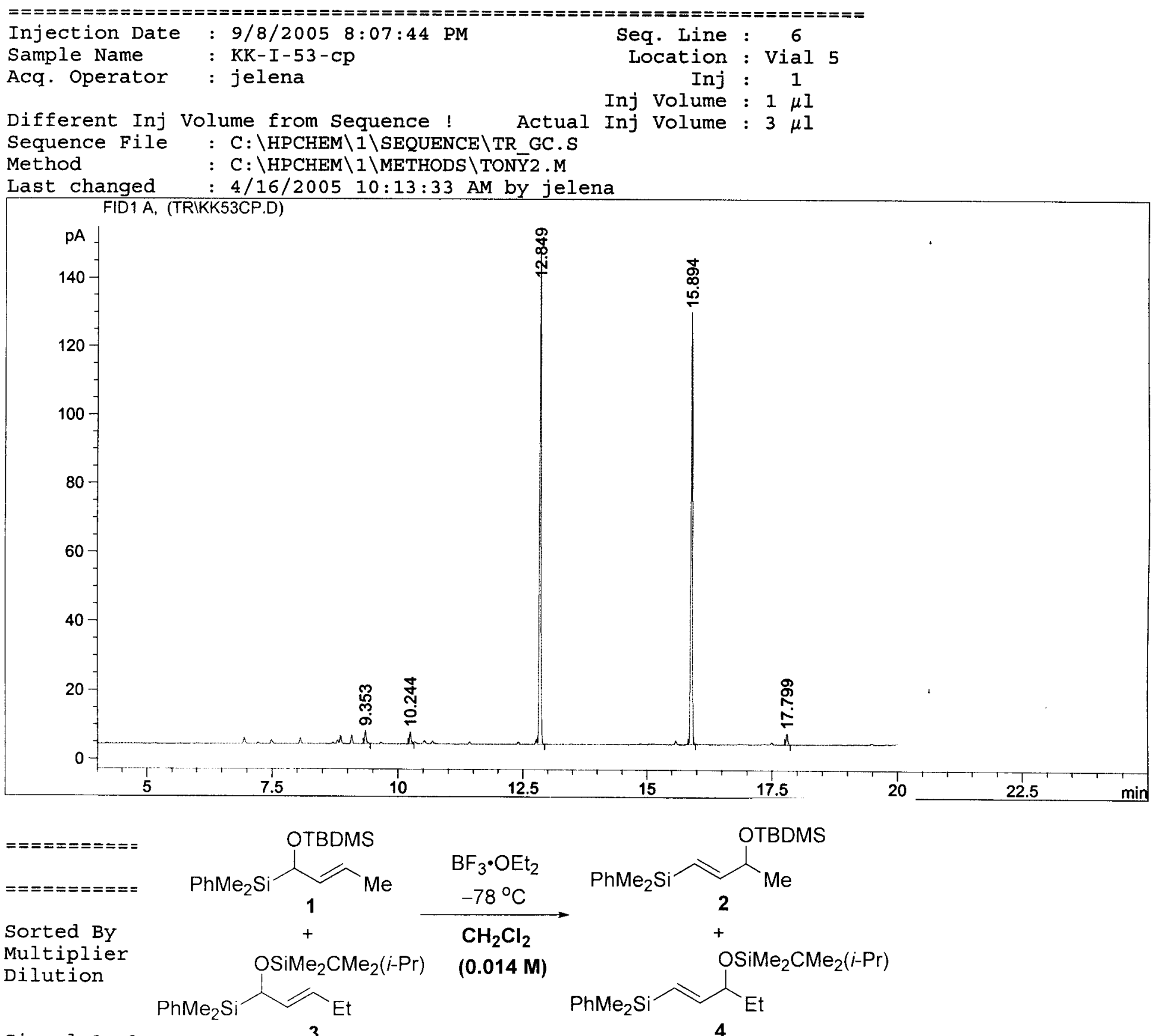

Signal 1: 1

\begin{tabular}{|c|c|c|c|c|c|c|}
\hline $\begin{array}{c}\text { Peak } \\
\#\end{array}$ & $\begin{array}{l}\text { RetTime } \\
\text { [min] }\end{array}$ & Type & $\begin{array}{l}\text { Width } \\
\text { [min] }\end{array}$ & $\begin{array}{r}\text { Area } \\
{\left[\mathrm{pA}{ }^{*} \mathrm{~s}\right]}\end{array}$ & $\begin{array}{l}\text { Height } \\
\text { [pA] }\end{array}$ & $\begin{array}{c}\text { Area } \\
\frac{8}{8}\end{array}$ \\
\hline & & & & & & $--\cdots--$ \\
\hline 1 & 1.425 & PB & 0.0116 & 8.39871 & 11.03529 & 1.32861 \\
\hline 2 & 1.989 & BB & 0.0158 & 4.94519 & 4.87553 & 0.78229 \\
\hline 3 & 2.590 & PB & 0.0199 & 10.48052 & 8.18075 & 1.65794 \\
\hline 4 & 3.319 & BB & 0.0230 & 23.23334 & 15.97332 & 3.67533 \\
\hline 5 & 9.353 & BB & 0.0351 & 8.92329 & 3.87690 & 1.41160 \\
\hline 6 & 10.244 & BV & 0.0348 & 8.28560 & 3.47881 & 1.31072 \\
\hline 7 & 12.849 & VB & 0.0296 & 294.95657 & 142.68611 & 46.65984 \\
\hline 8 & 15.894 & BB & 0.0327 & 265.79196 & 125.47290 & 42.04622 \\
\hline 9 & 17.799 & BB & 0.0281 & 7.12712 & 3.12608 & 1.12746 \\
\hline Total & & & & 632.14231 & 70570 & \\
\hline
\end{tabular}

Results obtained with enhanced integrator!

*** End of Report *** 

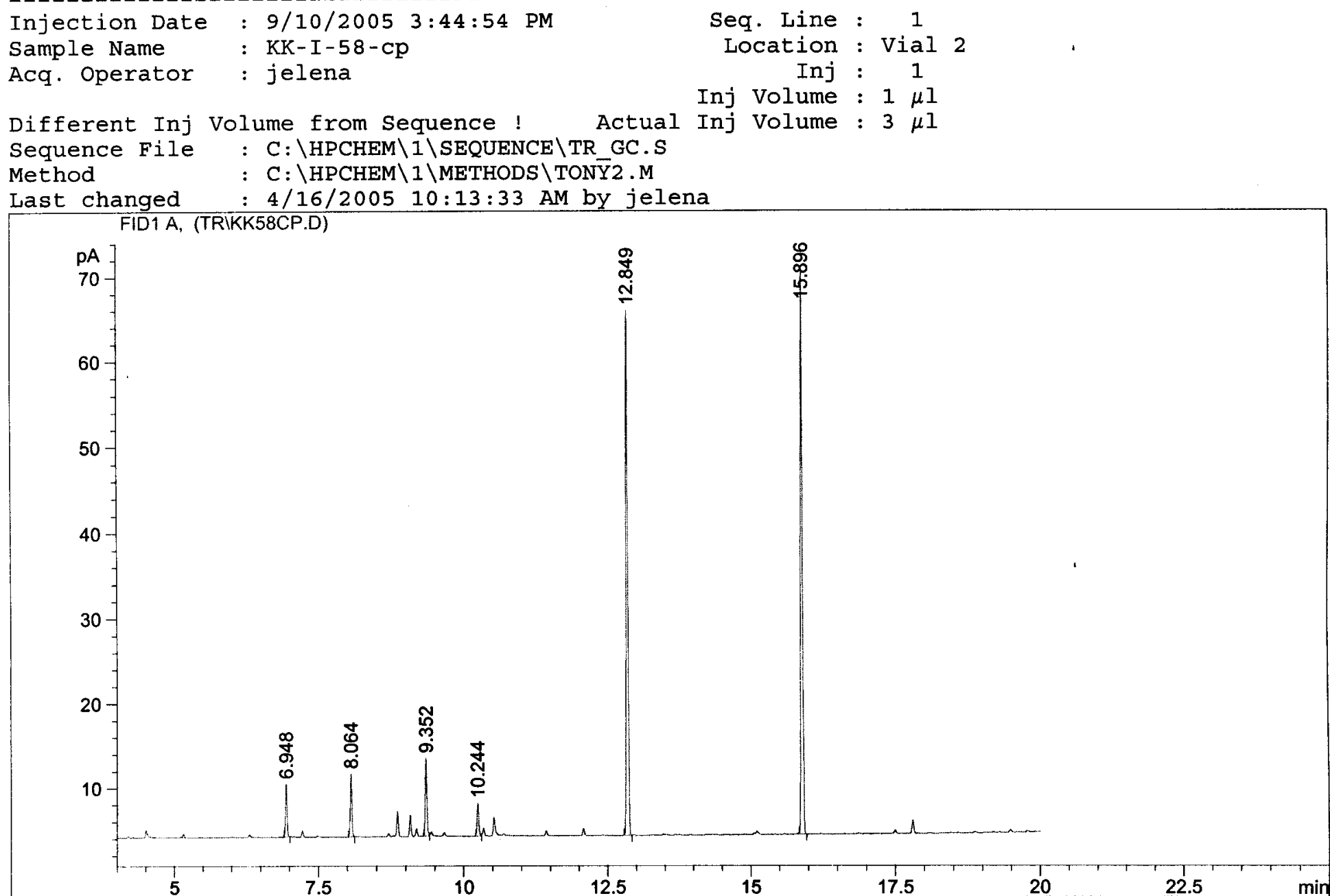

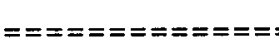

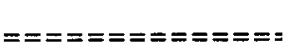

Sorted By

Multiplier

Dilution

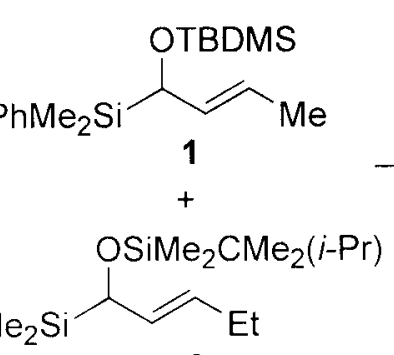

Signal 1: FID]

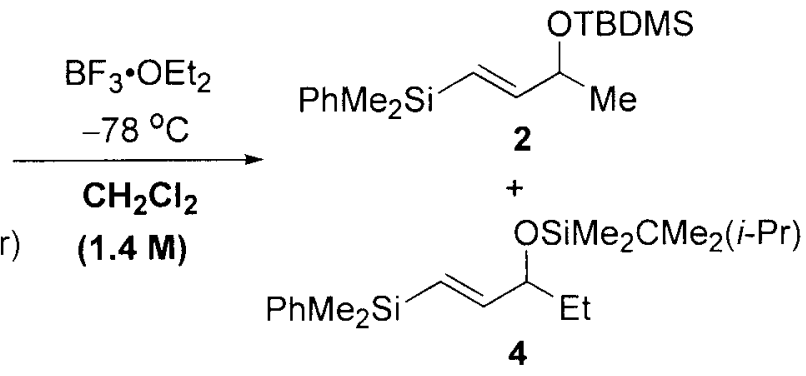

\begin{tabular}{|c|c|c|c|c|c|c|}
\hline $\begin{array}{c}\text { eak } \\
\#\end{array}$ & $\begin{array}{l}\text { RetTime } \\
\text { [min] }\end{array}$ & Type & $\begin{array}{c}\text { width } \\
\text { [min] }\end{array}$ & $\begin{array}{r}\text { Area } \\
{\left[\mathrm{pA}^{*} \mathrm{~s}\right]}\end{array}$ & $\begin{array}{l}\text { Height } \\
{[\mathrm{pA}]}\end{array}$ & $\begin{array}{c}\text { Area } \\
\frac{o}{b}\end{array}$ \\
\hline & & & & & & \\
\hline 1 & 1.426 & BB & 0.0119 & 14.97201 & 19.66622 & 3.78381 \\
\hline 2 & 1.991 & PV & 0.0157 & 8.93680 & & 2.25856 \\
\hline 3 & 2.591 & BV & 0.0195 & 14.77403 & 11.67691 & 3.73377 \\
\hline 4 & 3.320 & PB & 0.0223 & 33.93 & 23. & 8.57673 \\
\hline 5 & 6.948 & $\mathrm{BB}$ & 0.0 & 11.95 & 186 & 3.0 \\
\hline 6 & 8.064 & BB & 0.0 & 14.3 & 745 & 3.6 \\
\hline 7 & 9.352 & BV & 0.0 & 18.22 & 9. & 4.6 \\
\hline 8 & 10.244 & BB & 0.0 & & 3.87595 & 2.04068 \\
\hline 9 & 12.849 & BB & 0.0329 & 131.30743 & 61.57432 & 33.18472 \\
\hline 10 & 15.896 & BB & 0.0293 & 139.19469 & 66.24973 & 35.17803 \\
\hline
\end{tabular}

Totals :

$395.68638 \quad 217.97758$

Results obtained with enhanced integrator! 

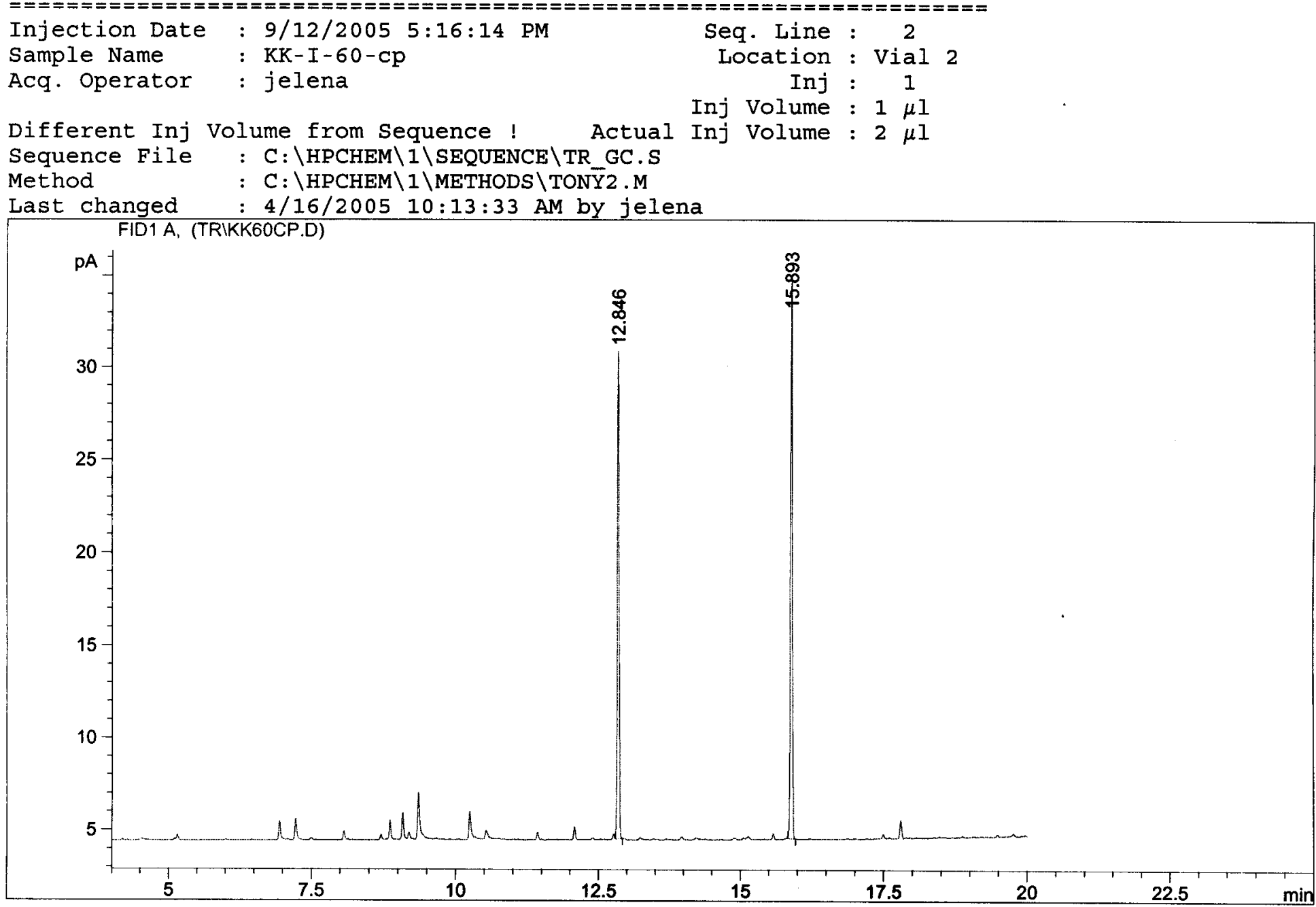

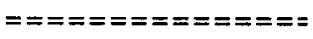

$===+====+====$

Sorted By Multiplier OTBDMS Dilution

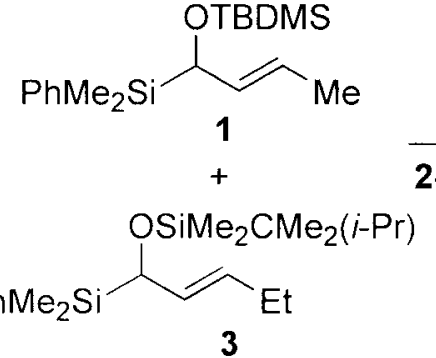<smiles>CCO[SbH](OC)OC</smiles>
$-78^{\circ} \mathrm{C}$<smiles>CC(C)[O+]C=C[SiH2]C(C)C</smiles>

Signal 1: FIDI

$(0.14 \mathrm{M})$

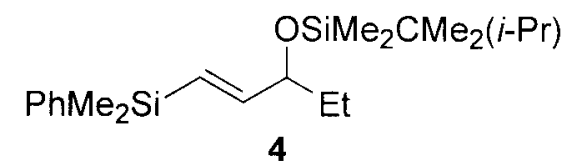

Totals :

$177.11827 \quad 105.69225$

Results obtained with enhanced integrator! 


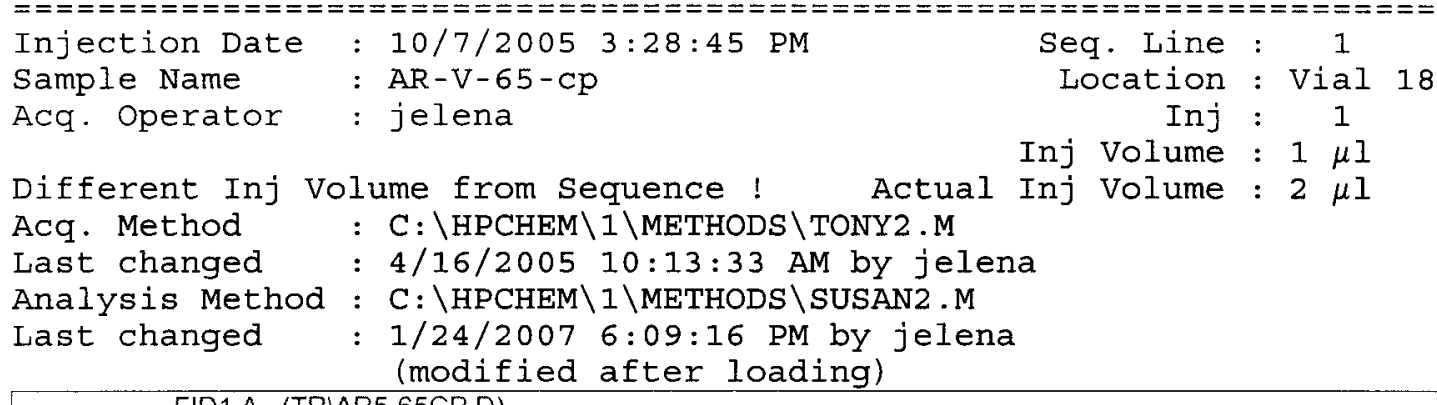

Inj Volume : $1 \mu 1$

Different Inj Volume from Sequence ! Actual Inj Volume : $2 \mu 1$

Acq. Method : C: $\backslash$ HPCHEM $\backslash 1 \backslash$ METHODS $\backslash$ TONY2.M

Last changed : 4/16/2005 10:13:33 AM by jelena

Analysis Method : C: $\backslash$ HPCHEM $\backslash 1 \backslash$ METHODS $\backslash$ SUSAN2.M

Last changed : 1/24/2007 6:09:16 PM by jelena (modified after loading)
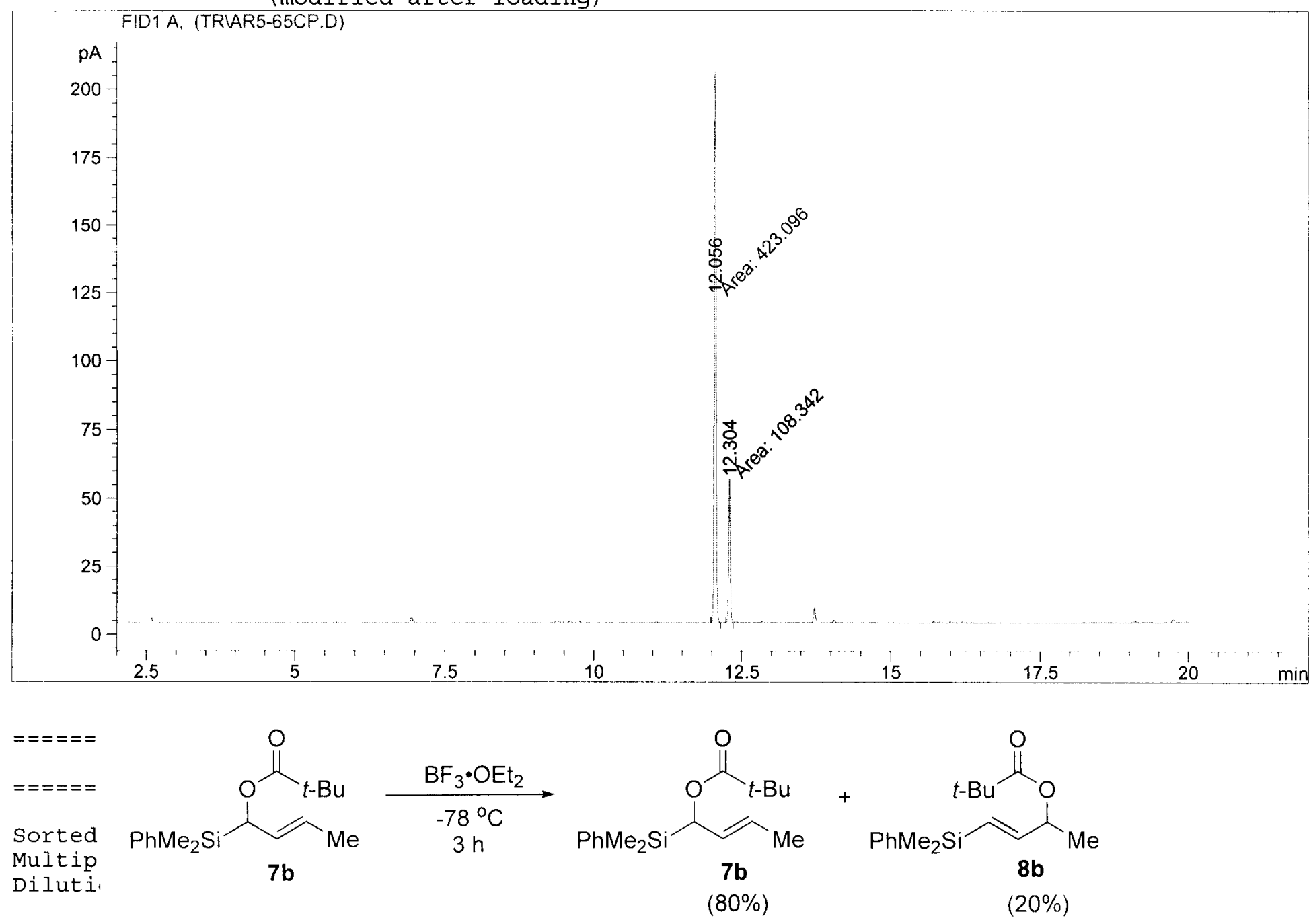

Signal 1: FID1 A,

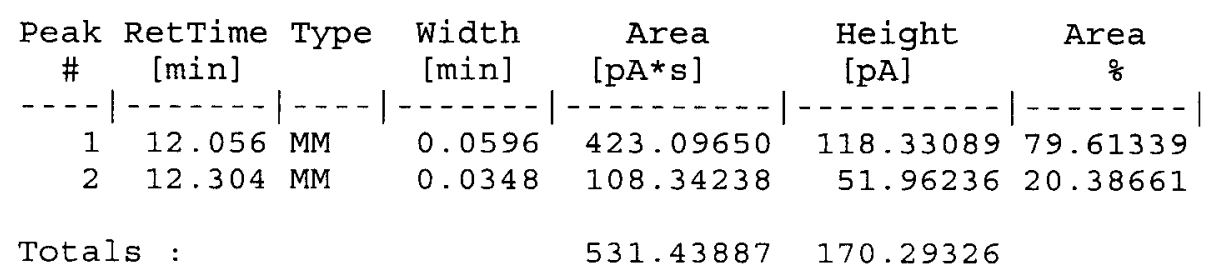

Results obtained with enhanced integrator! 


-1
0
0
0
0
0
0
0
0
0
0
-1
$\ddot{0}$
0
0
2
0
0
-1
0
0
0
0
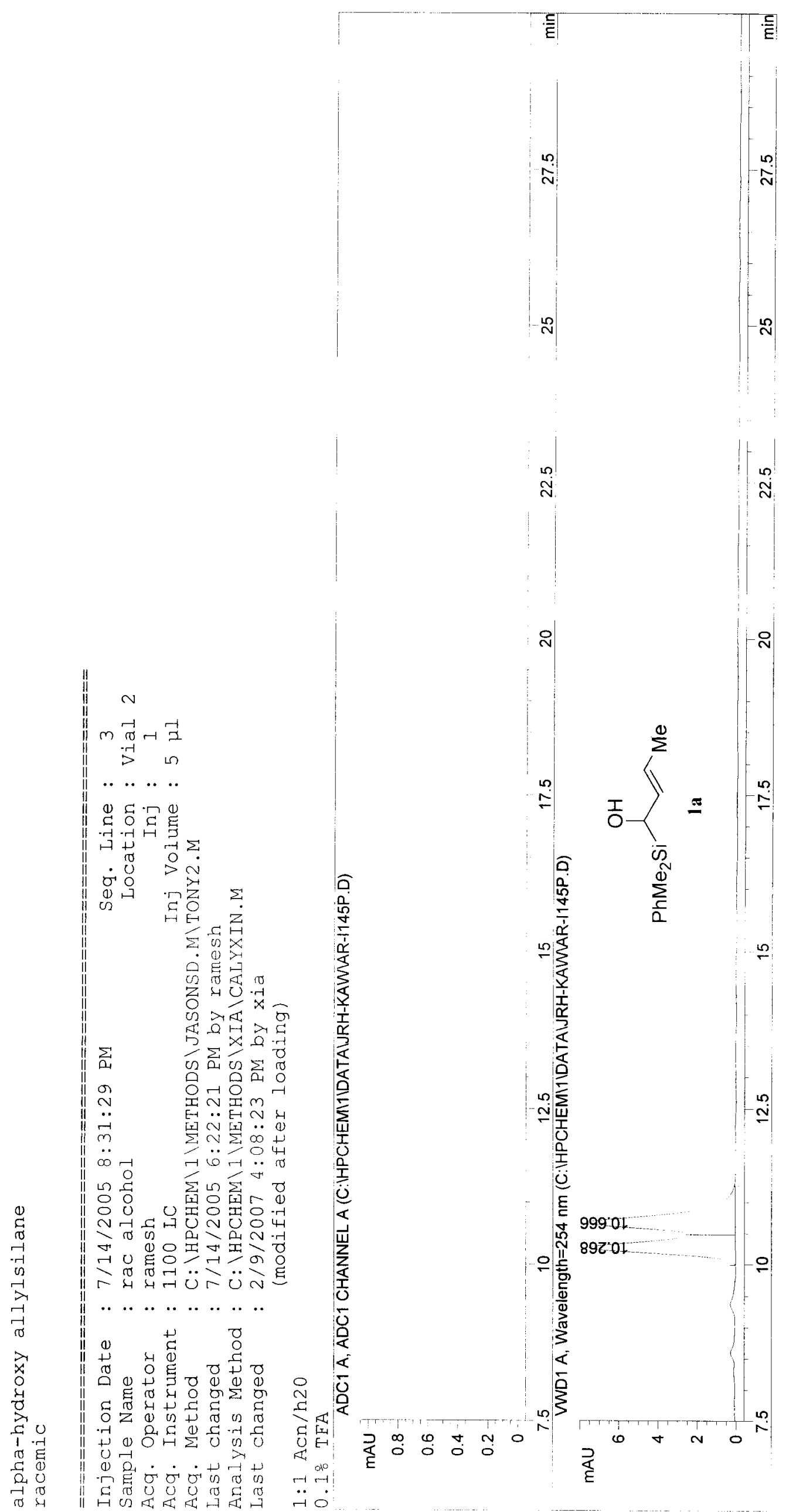


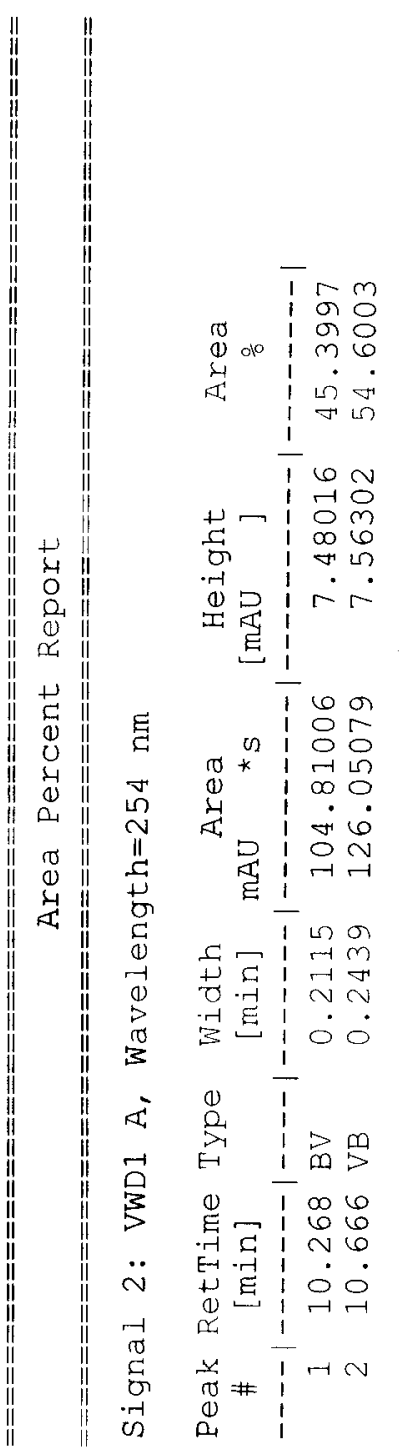

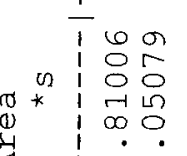

द

密

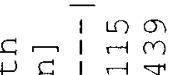

गु.

苛圭

(1) $\frac{1}{1}$

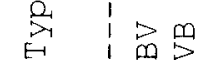

( T) 0

E马 100

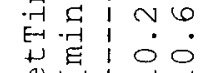

의 음으

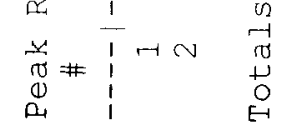

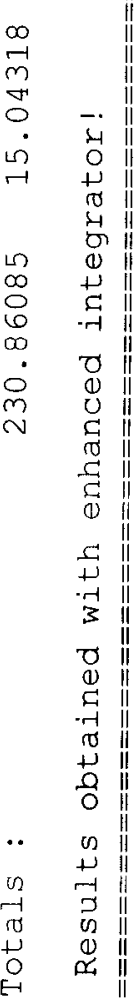




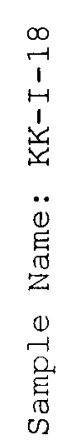

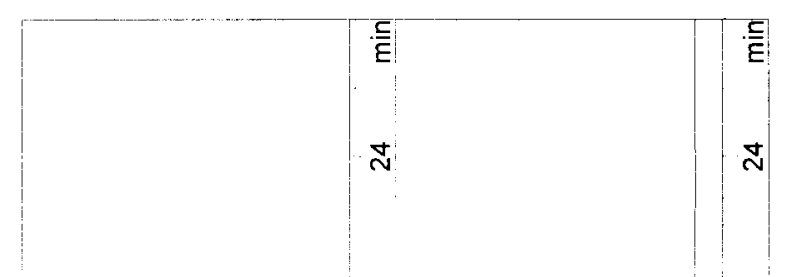

N

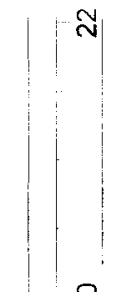

용
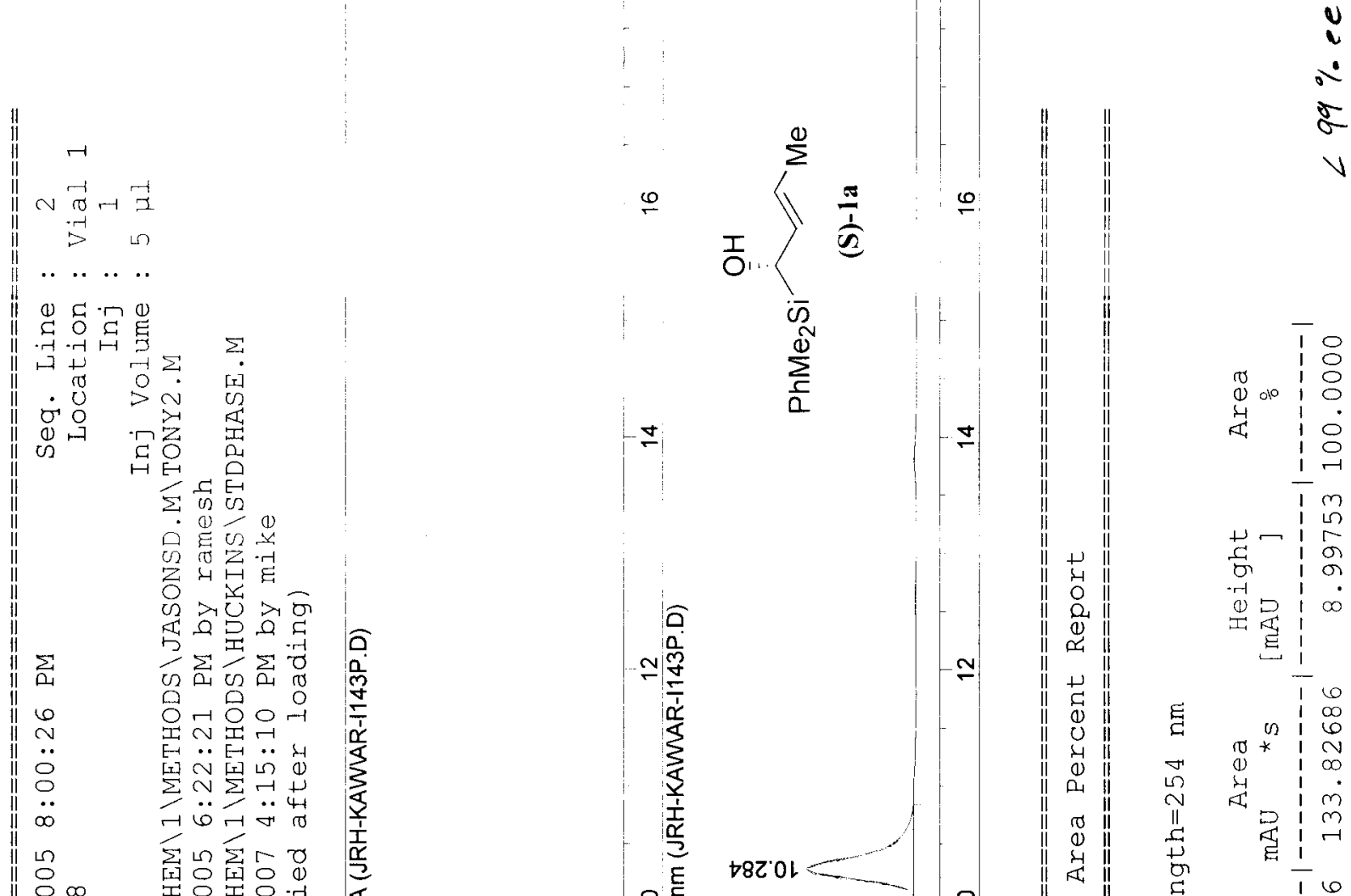

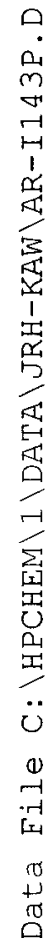

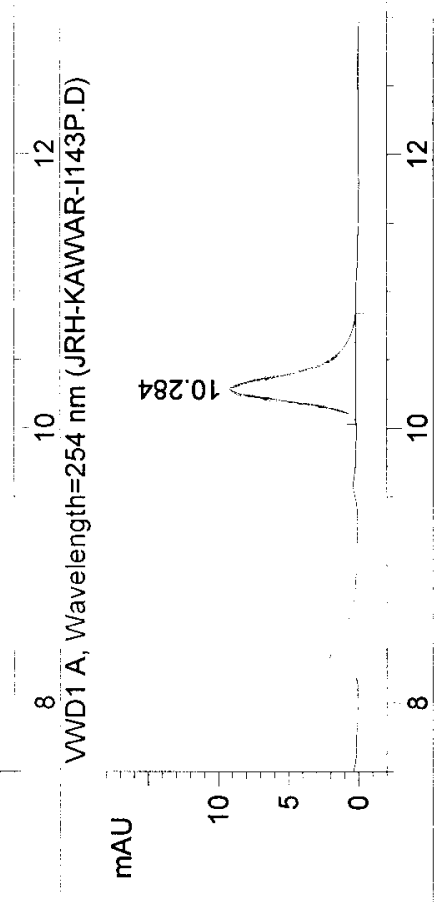

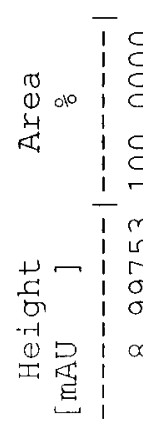

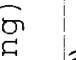

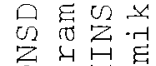

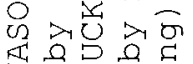

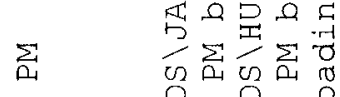

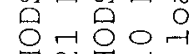

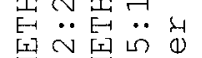

Nㅗㄴㄷㅏ

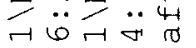

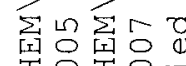

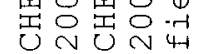

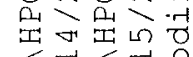

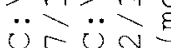

空离

OrUn

$\cdot . \cdot$

¿ $\overline{0}$

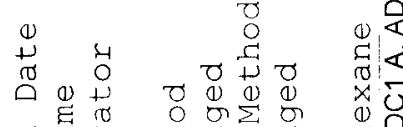

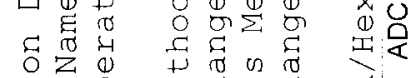

तो

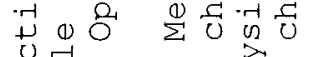

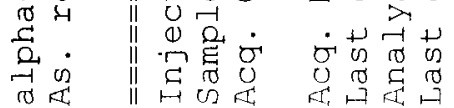

岁
id
$m$

롱ㅇ $\infty$

है

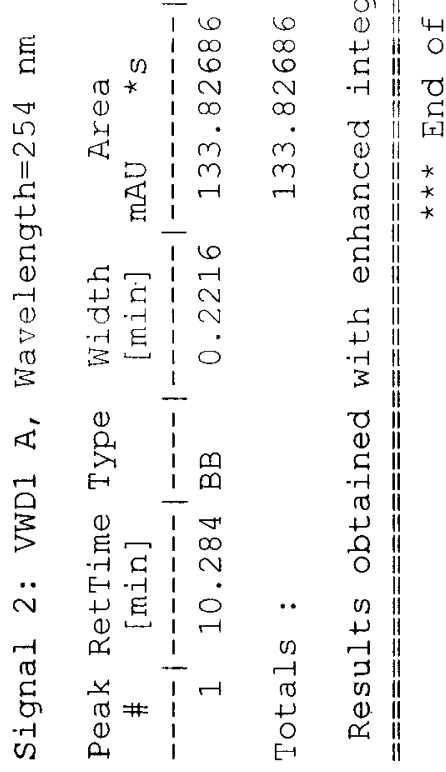


Data File C: \CHEM32\1\DATA \NAPPY KKRACASA2.D

Sample Name: racallylsilane

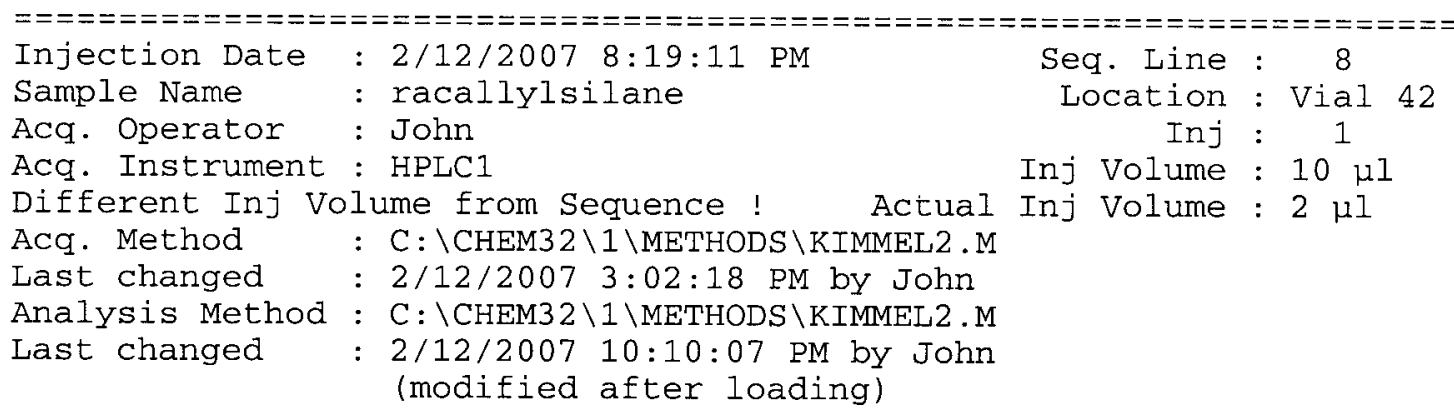
(modified after loading)

99:1 Hexanes: IPA

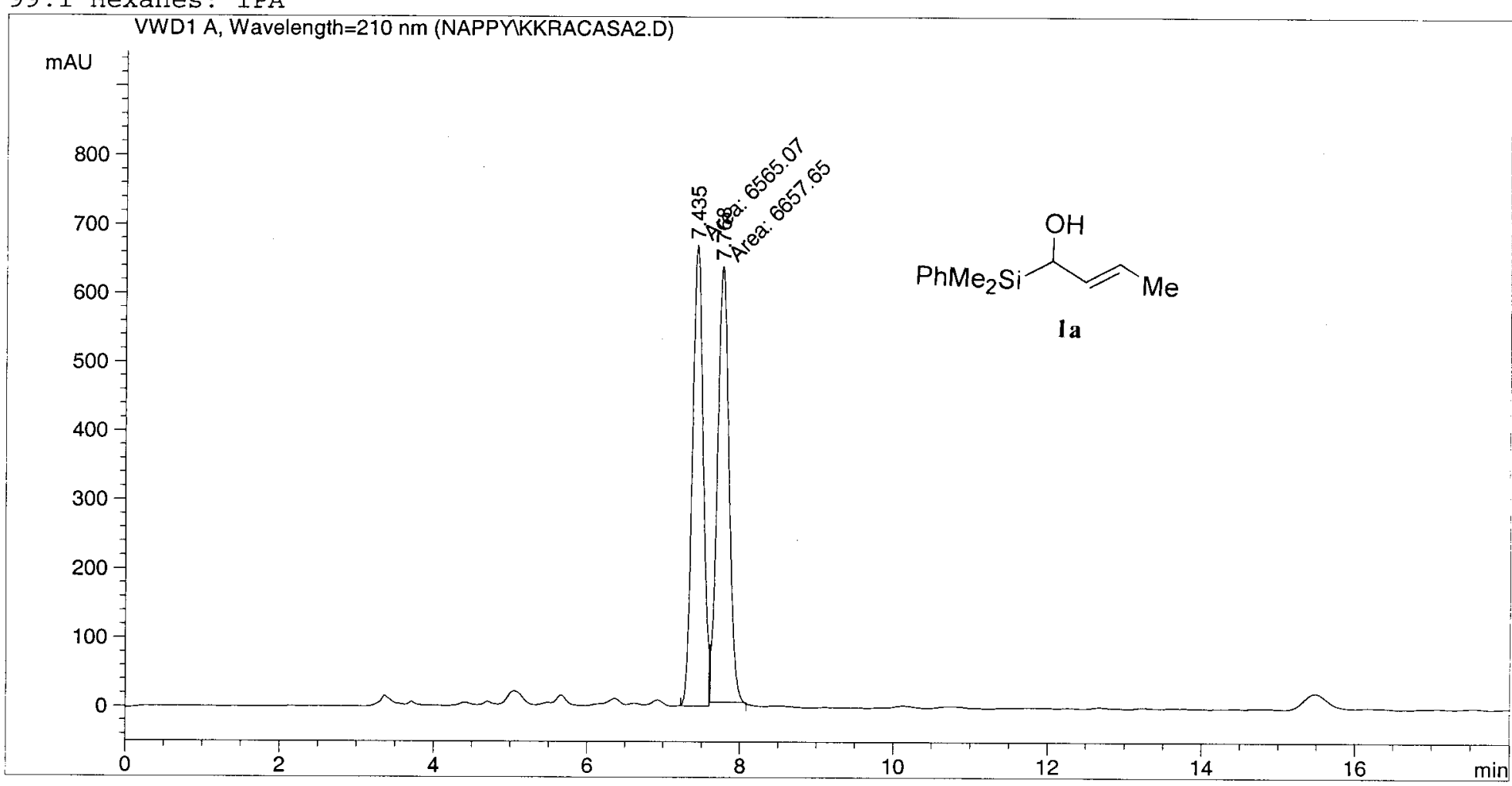

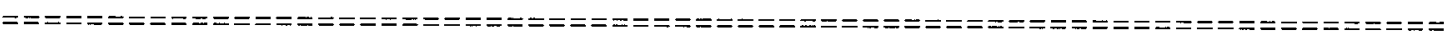

Area Percent Report

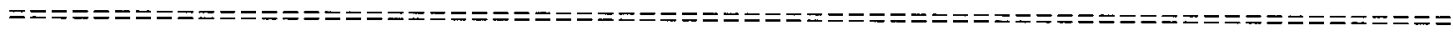

Sorted By

Multiplier

Dilution

Signal

1.0000

Use Multiplier \& Dilution Factor with ISTDs

Signal 1: VWD1 A, Wavelength=210 nm

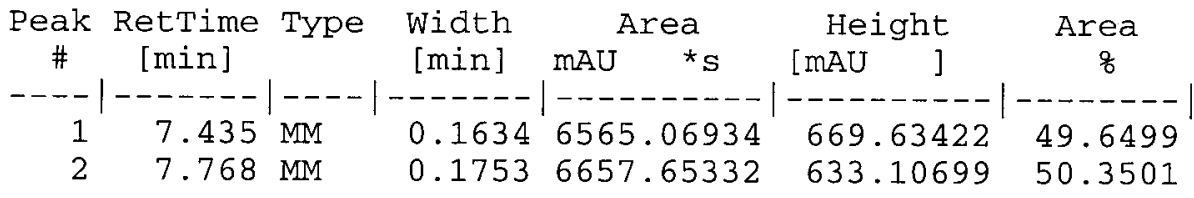

Totals : $\quad 1.32227 \mathrm{e} 4 \quad 1302.74121$ 
Data File C: \CHEM32 \1\DATA \NAPPY KK135PA2 .D

Sample Name: KK-I-135-P
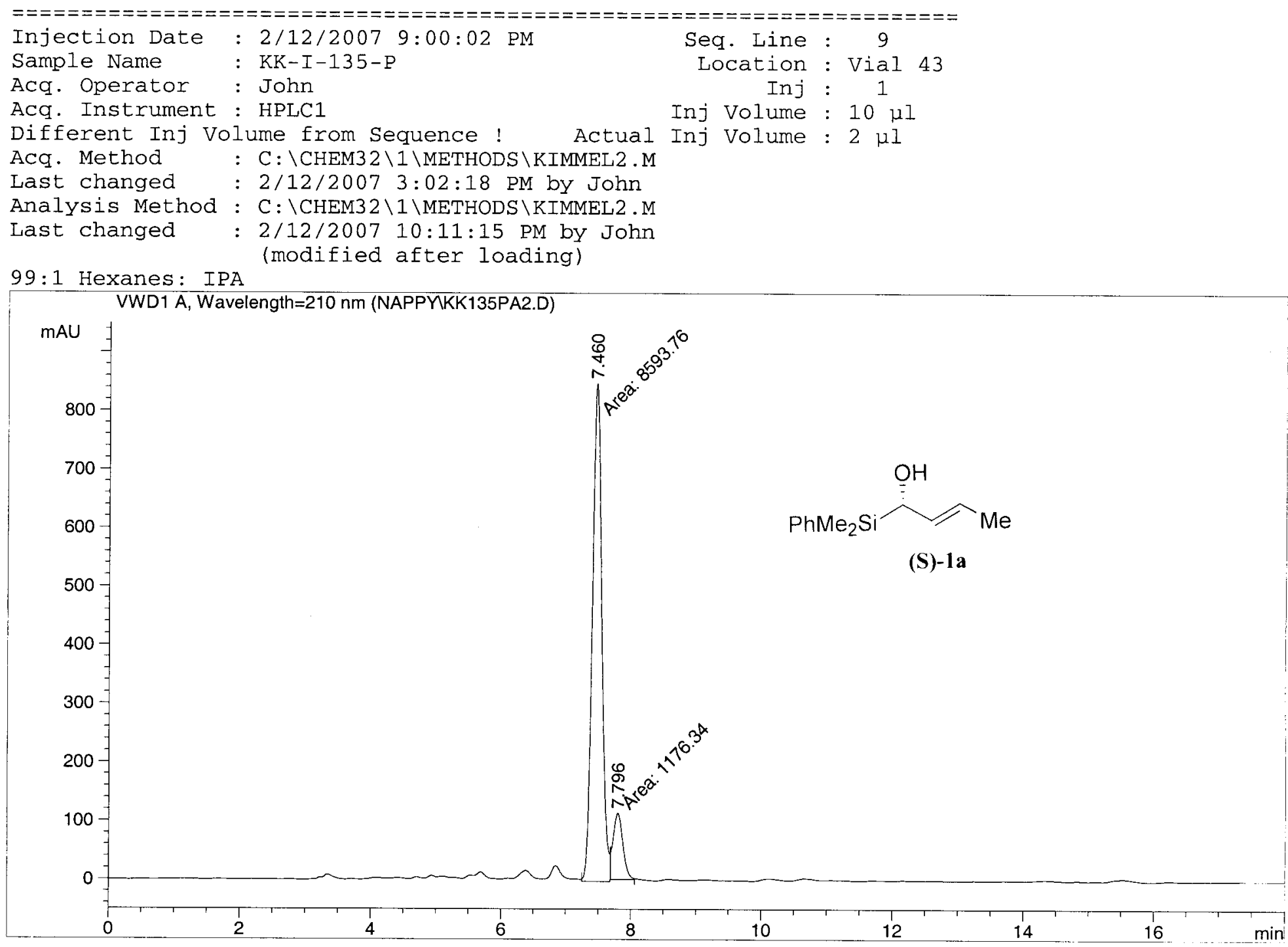

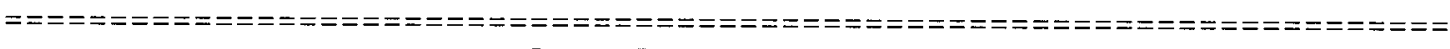

Area Percent Report

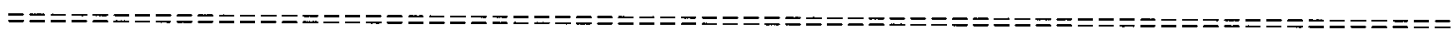

Sorted By

Multiplier

Dilution

Signal

1.0000

Use Multiplier \& Dilution Factor with ISTDs

Signal 1: VWD1 A, Wavelength=210 nm

\begin{tabular}{|c|c|c|c|c|c|c|c|}
\hline Peak & RetTime & Type & Width & Area & Height & Area & \\
\hline \# & [min] & & [min] & $\mathrm{mAU} \quad{ }^{*} \mathrm{~s}$ & {$[\mathrm{mAU} \quad]$} & q & \\
\hline 1 & 7.460 & $\mathrm{MM}$ & 0.1684 & 8593.75684 & 850.41638 & 87.9598 & \\
\hline 2 & 7.796 & MM & 0.1734 & 1176.34094 & 113.03912 & 12.0402 & $76 \%$ ee \\
\hline Total & ls & & & 9770.09778 & 963.45551 & & \\
\hline
\end{tabular}



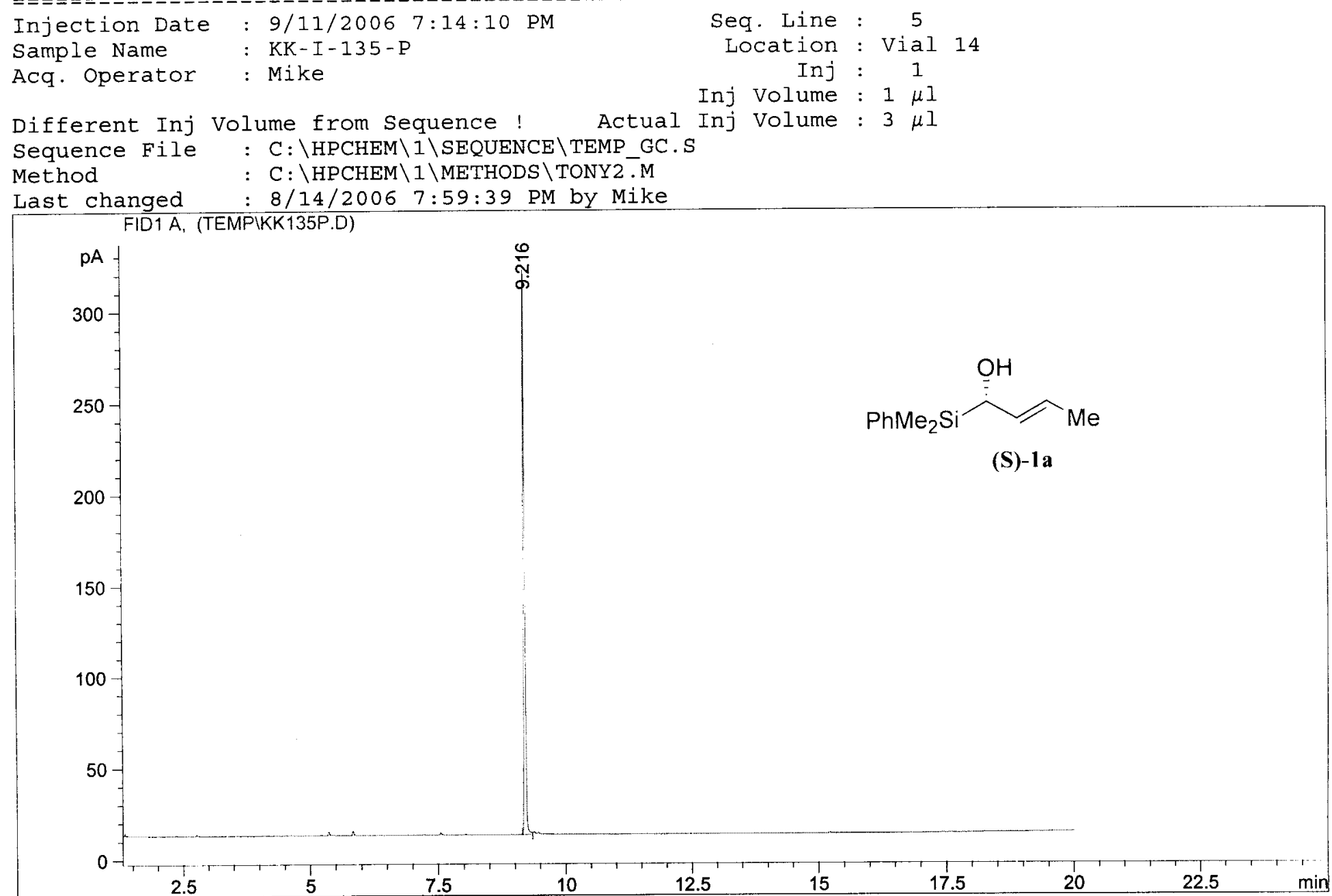

Area Percent Report

\begin{tabular}{|c|c|c|}
\hline Sorted By & $:$ & Signal \\
\hline Multiplier & : & 1.0000 \\
\hline Dilution & : & 1.0000 \\
\hline
\end{tabular}

Signal 1: FID1 A,

\begin{tabular}{|c|c|c|c|c|c|c|}
\hline $\begin{array}{c}\text { Peak } \\
\#\end{array}$ & $\begin{array}{c}\text { RetTime } \\
\text { [min] }\end{array}$ & Type & $\begin{array}{c}\text { Width } \\
\text { [min] }\end{array}$ & $\begin{array}{r}\text { Area } \\
{\left[\mathrm{pA}^{*} \mathrm{~s}\right]}\end{array}$ & $\begin{array}{l}\text { Height } \\
{[\mathrm{pA}]}\end{array}$ & $\begin{array}{c}\text { Area } \\
\frac{o}{8}\end{array}$ \\
\hline 1 & 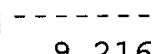 & & $-\overline{x-1}-\overline{0}$ & $613 \quad 38043$ & 30841422 & 10002 \\
\hline 1 & 9.210 & DV & 0.030 & 013.30043 & & \\
\hline Total & Ls & & & 613.38043 & 308.41422 & \\
\hline
\end{tabular}

Results obtained with enhanced integrator!

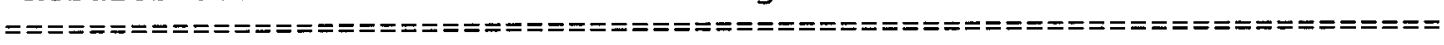

*** End of Report *** 


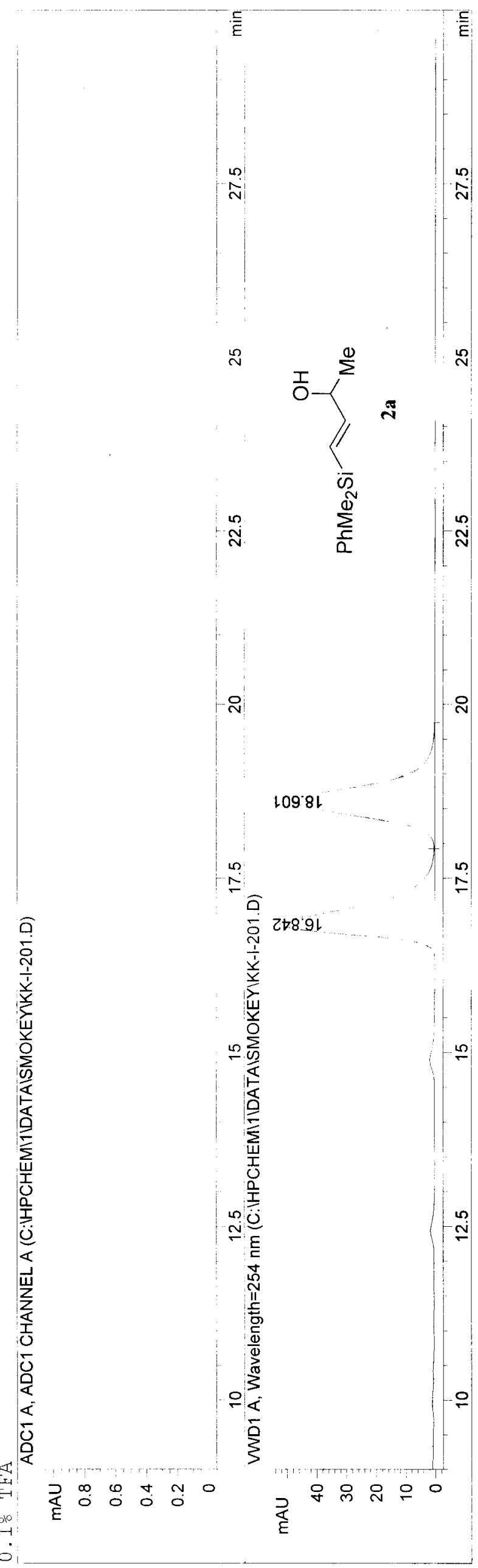

욱

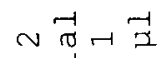

$>$ in

........

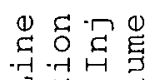

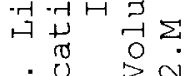

审,

空

它总家

$\sum_{0}=40-1$

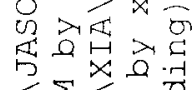

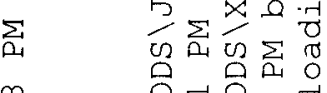

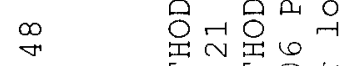

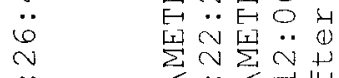

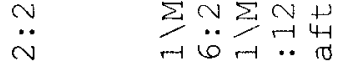

n $\quad \sum_{i=1}^{\infty} \sum_{0}^{+\infty}$

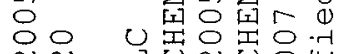

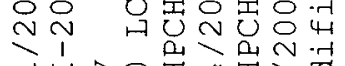

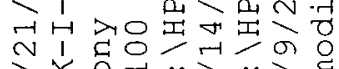

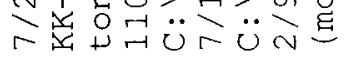

........

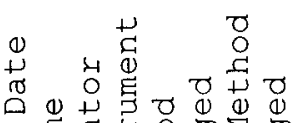

व

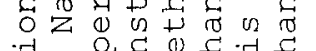

- $\begin{aligned} & 0 \\ & 0\end{aligned}$

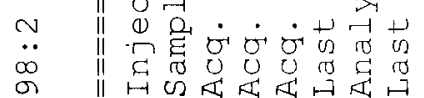
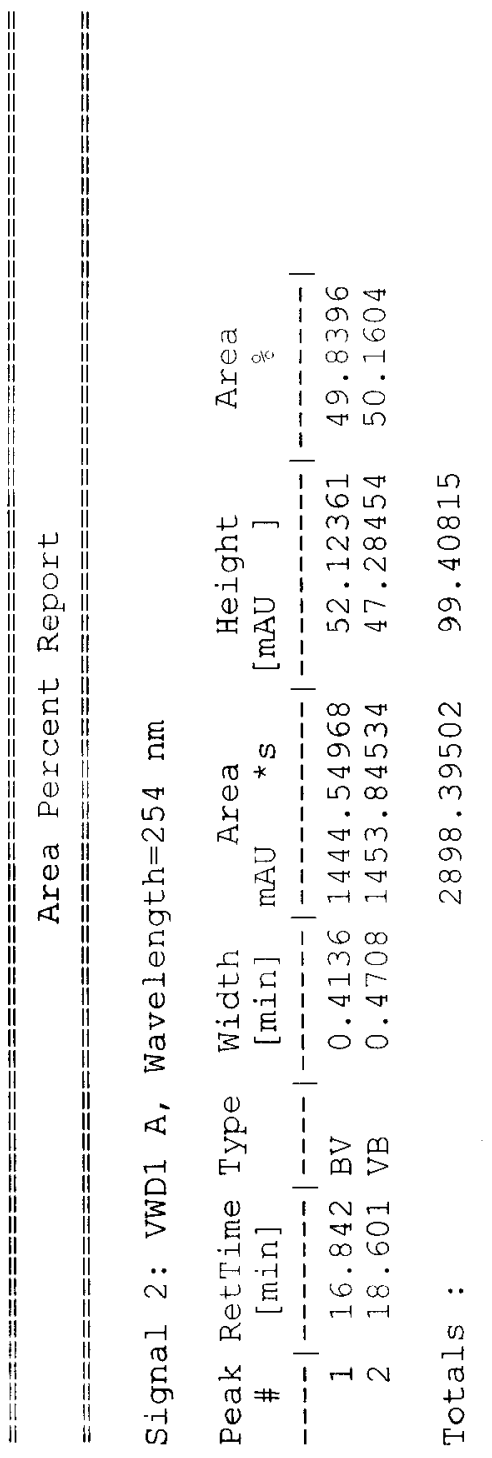

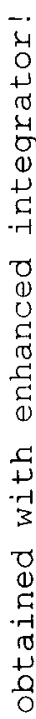




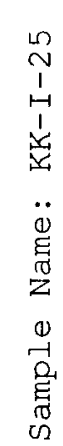
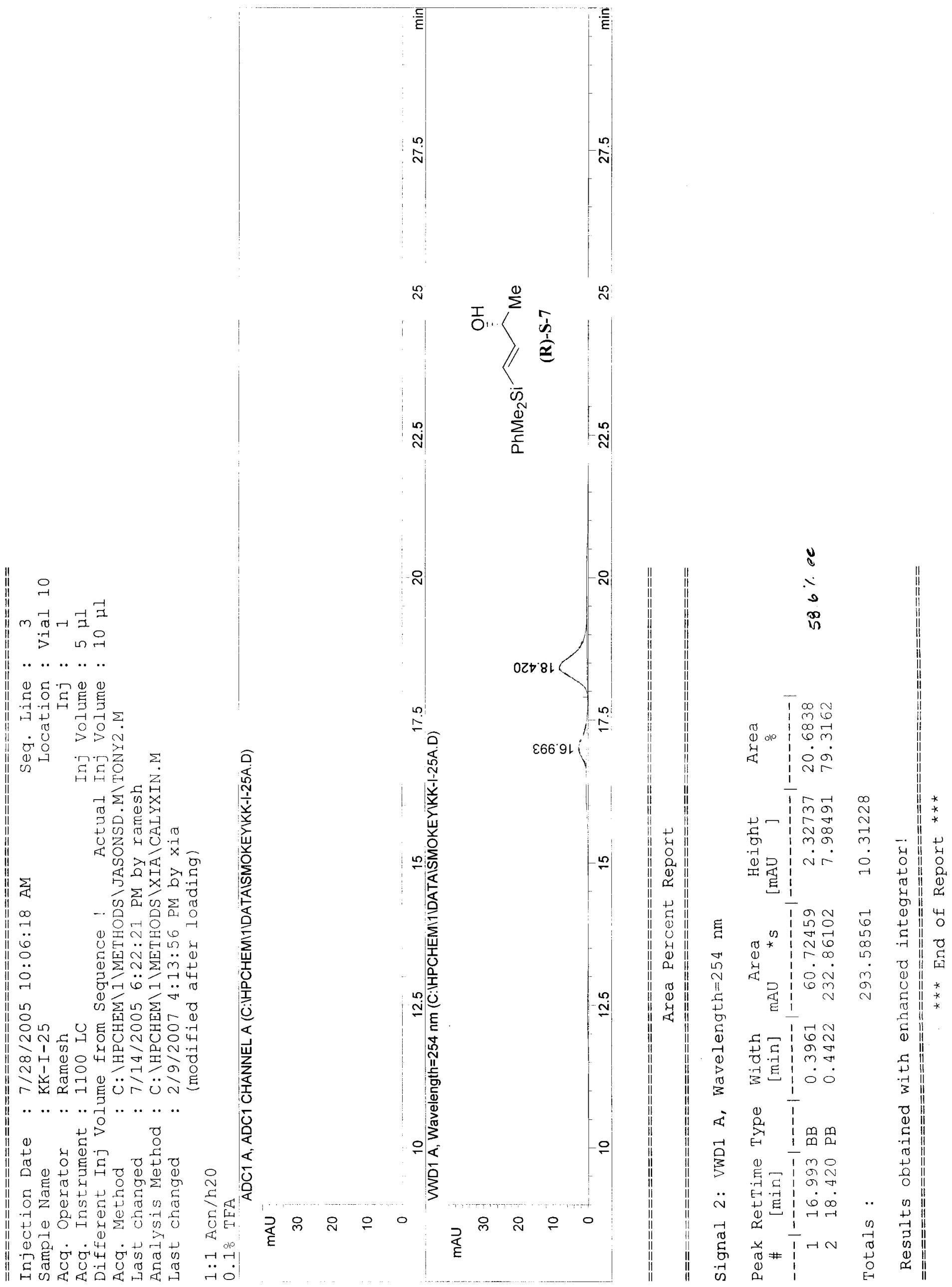

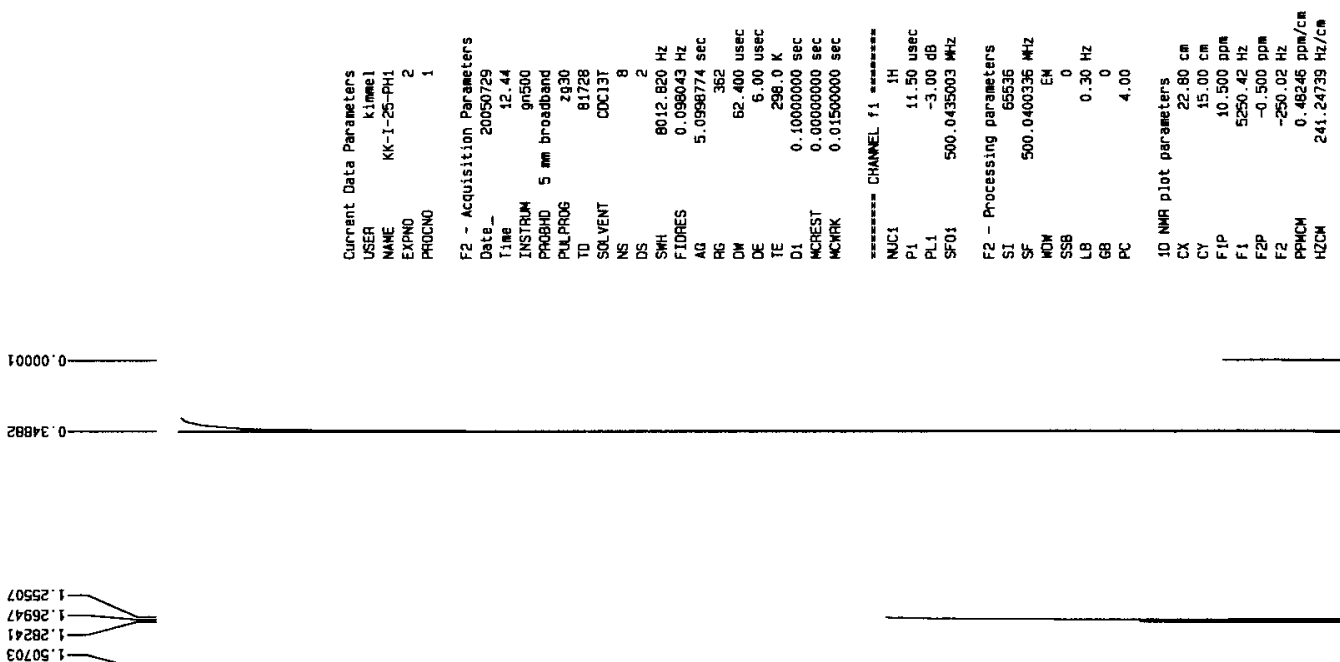

schs':

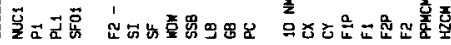

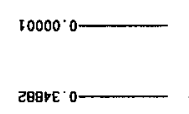

(2टाE

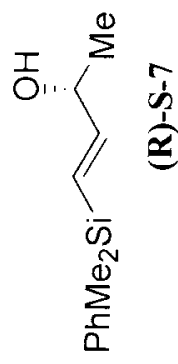

ES0S6:2-

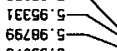

$96066 \cdot 5$

OTEPI.9-

G5081.9- $\cdot 9$

$6 \varepsilon 061 \cdot 9$

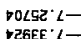

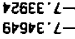

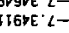

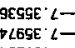

geors 2

$9025 S^{2}:$

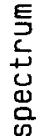

홓

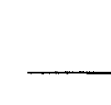

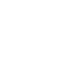

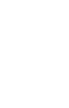
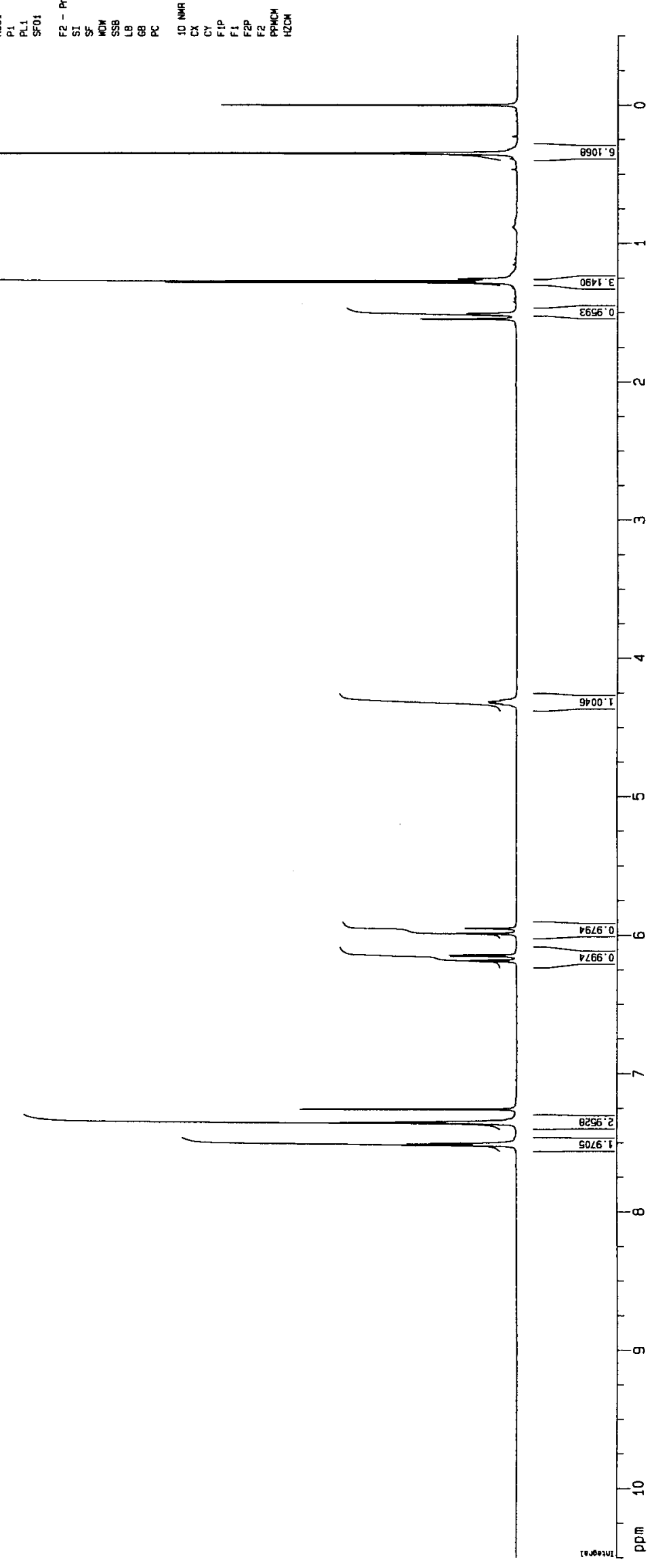

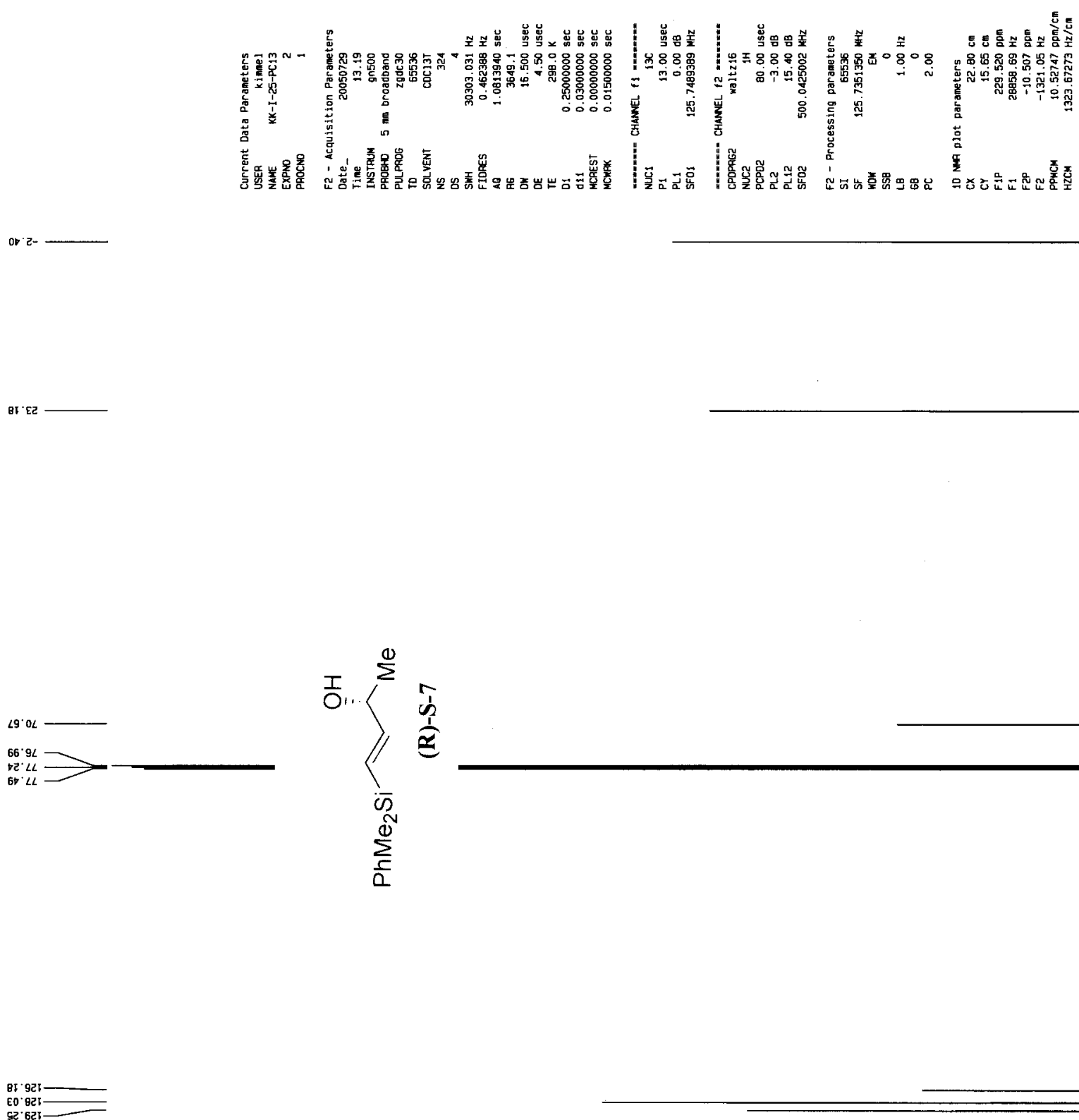

с9ट $621-$

EO' $\mathrm{E} \varepsilon$

sg' เ9i

$\hat{\frac{1}{s}}$

$\sum_{\frac{\pi}{\alpha}}^{\infty}$

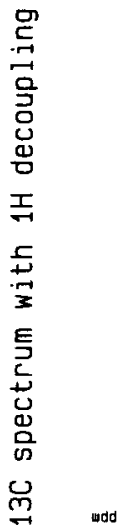


Date: Sat, Oct 21, 2006 8:09 PM

Data: rac vinylsilane 3

Sampling Int: 0.1 Seconds

Data:<smiles>CC(O)C=C[SiH2]C(C)P</smiles>

$2 a$

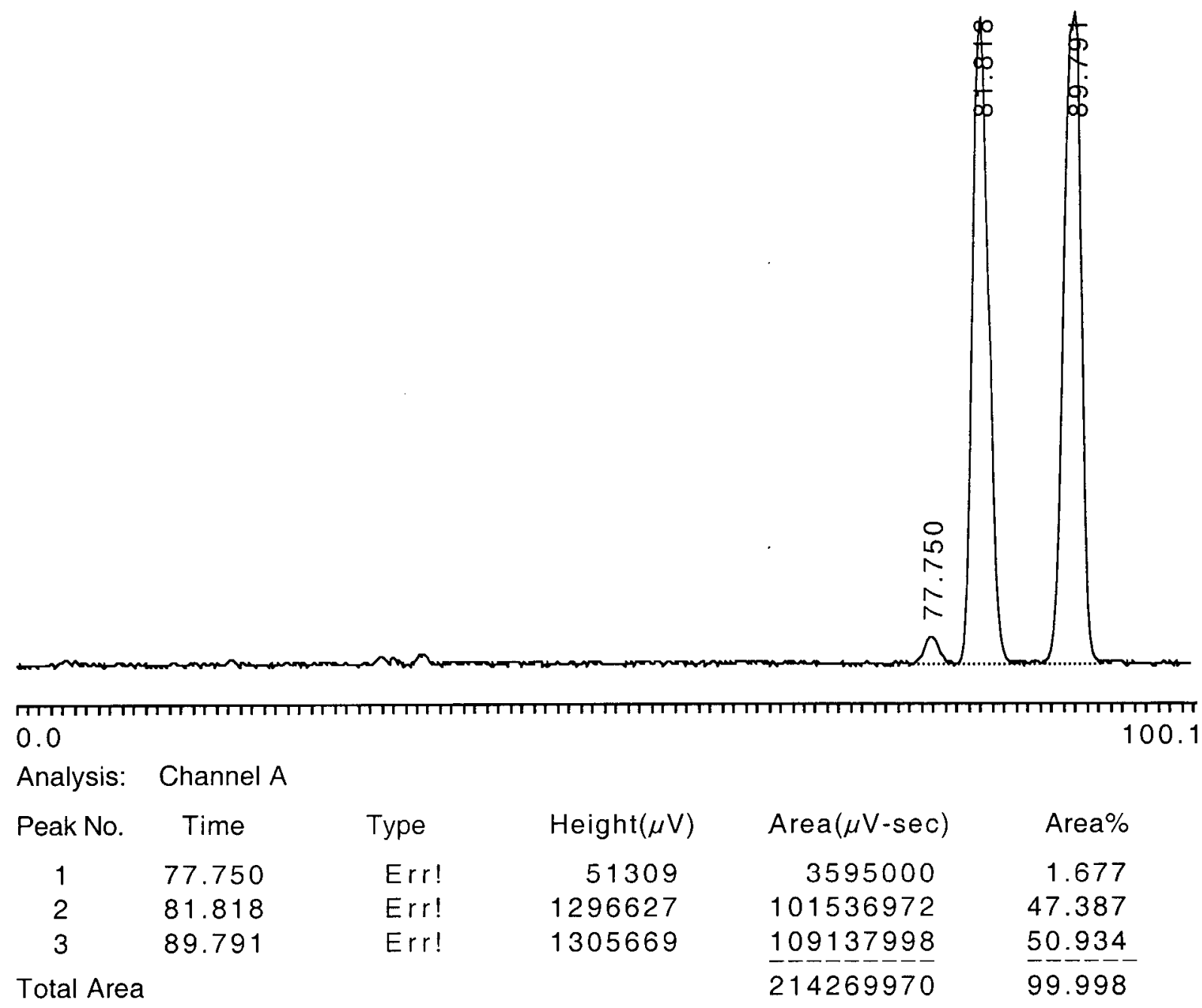


Date: Sat, Oct 21, 2006 10:11 PM

Data: KK-I-128-P-4

Processing File:

Sampling Int: 0.1 Seconds

Data:<smiles>C[C@H](O)/C=C/[SiH2][Na]</smiles>

(R)-S-7

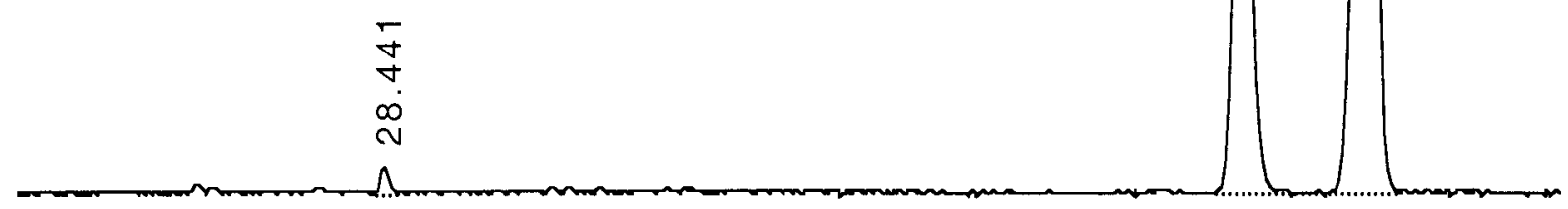

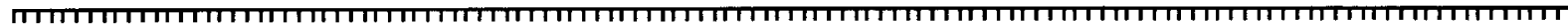

0.0

120.0

Analysis: Channel A

\begin{tabular}{cccrrrr} 
Peak No. & Time & Type & Height $(\mu \mathrm{V})$ & Area $(\mu \mathrm{V}$-sec $)$ & \multicolumn{1}{c}{ Area\% } \\
1 & 28.441 & Err! & 49905 & 1487089 & 0.741 & \\
2 & 94.658 & N4 & 783894 & 63913648 & 31.877 & $35.5 \%$ ee \\
3 & 104.588 & Err! & 1532838 & 135098168 & 67.381 & \\
\multicolumn{2}{l}{ Total Area } & & & 200498905 & 99.999
\end{tabular}




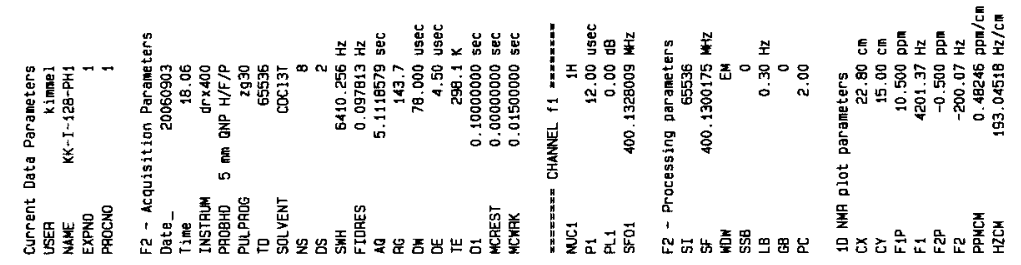

OORE' 0 
$98: 2$ hexanes:iPA, $0.8 \mathrm{~mL} / \mathrm{min}$.

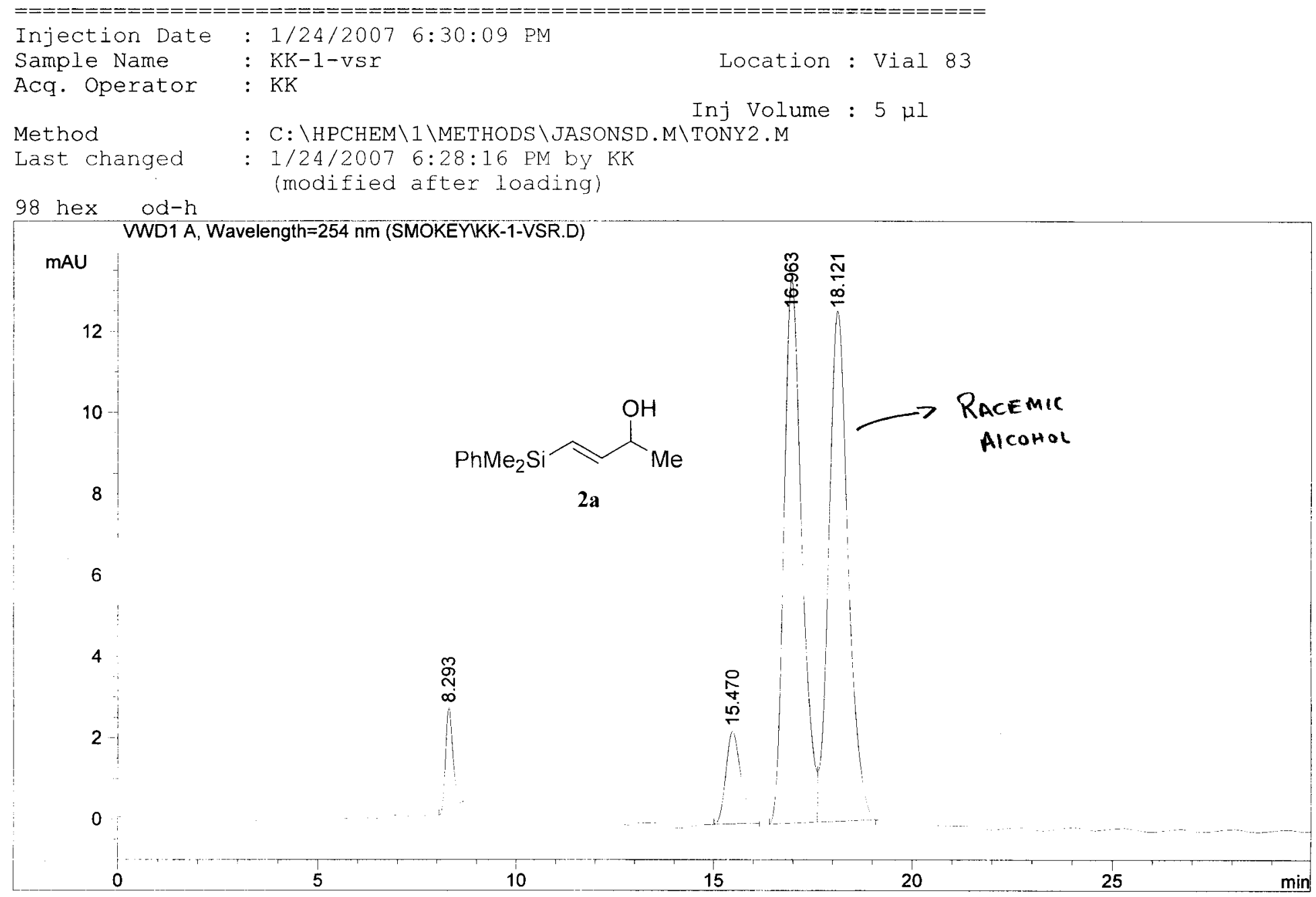

Area Percent Report

Sorted By

Multiplier

Dilution

Signal

1.0000

1.0000

Use Multiplier \& Dilution Factor with ISTDS

Signal 1: VWD1 A, Wavelength=254 nm

\begin{tabular}{|c|c|c|c|c|c|c|}
\hline \multirow{2}{*}{$\begin{array}{c}\text { Peak } \\
\#\end{array}$} & \multirow{2}{*}{$\begin{array}{c}\text { RetTime } \\
\text { [min] }\end{array}$} & \multirow[t]{2}{*}{ Type } & \multirow{2}{*}{$\begin{array}{l}\text { Width } \\
\text { [min] }\end{array}$} & Area & Height & \multirow{2}{*}{$\begin{array}{c}\text { Area } \\
\frac{0}{5}\end{array}$} \\
\hline & & & & $\mathrm{mAU} \quad{ }^{*} \mathrm{~s}$ & {$[\mathrm{mAU} \quad]$} & \\
\hline 1 & 8.293 & $\mathrm{~PB}$ & 0.1938 & 31.70396 & 2.51254 & 3.3814 \\
\hline 2 & 15.470 & $\mathrm{BB}$ & 0.4063 & 61.90915 & 2.28529 & 6.6030 \\
\hline 3 & 16.963 & $B V$ & 0.4759 & 419.38284 & 13.39424 & 44.7295 \\
\hline 4 & 18.121 & VP & 0.5135 & 424.60135 & 12.56973 & 45.2861 \\
\hline 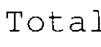 & & & & 937.59731 & 30. & \\
\hline
\end{tabular}

Results obtained with enhanced integrator! 


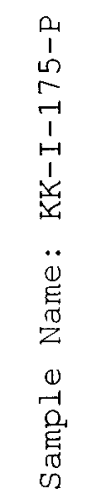

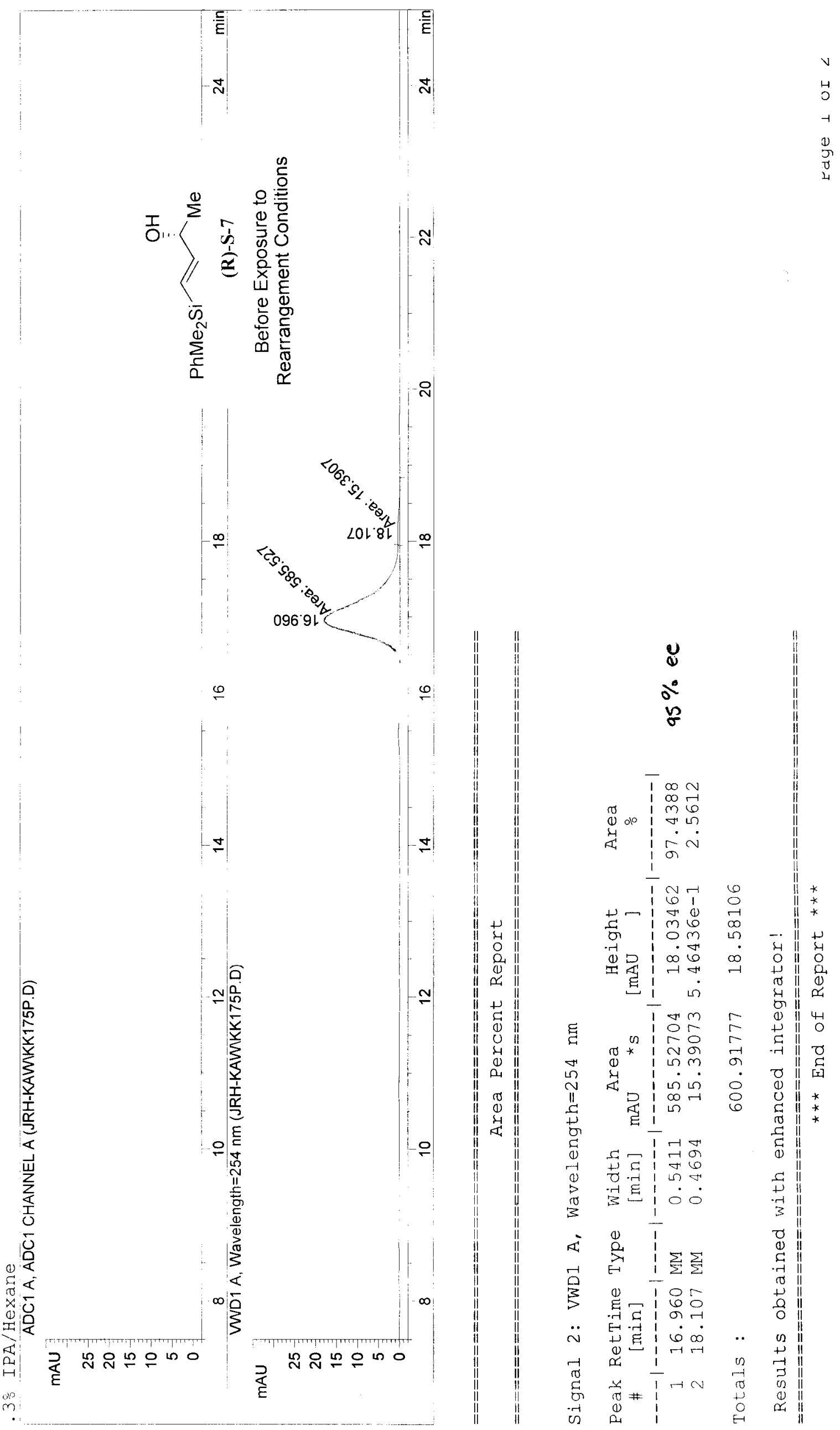




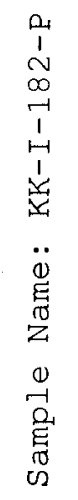

贷

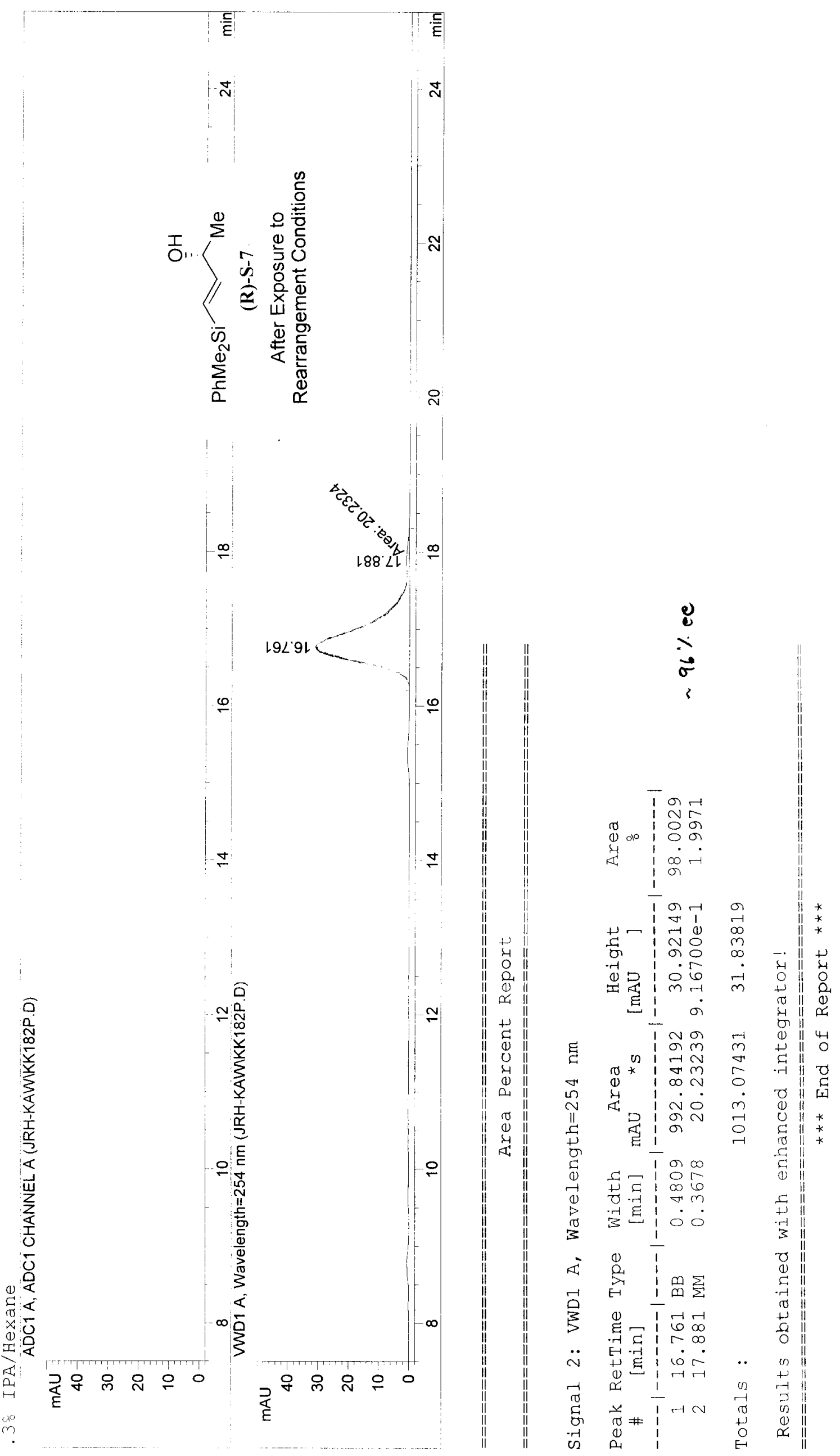

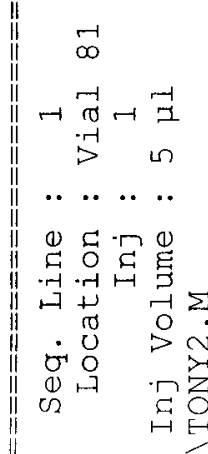

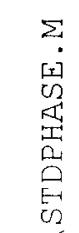

in

足要

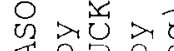

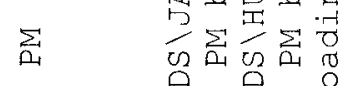

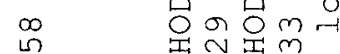

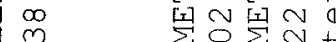

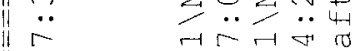

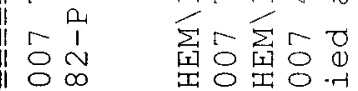

空造

$\ddot{u}+\ddot{u}$ 跑

... ..

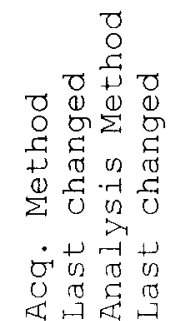

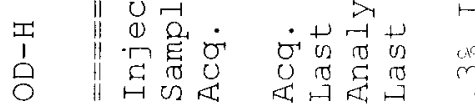

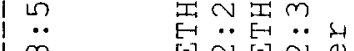

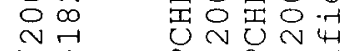

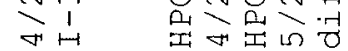

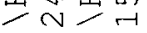

듵

要

10

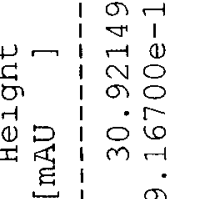

n 1 \%

(1)

ช . त का

$\stackrel{\square}{2}$

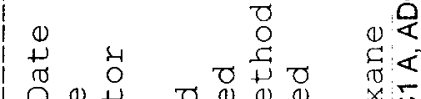

口

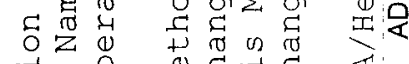

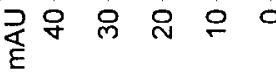




\section{Conformational Calculations}

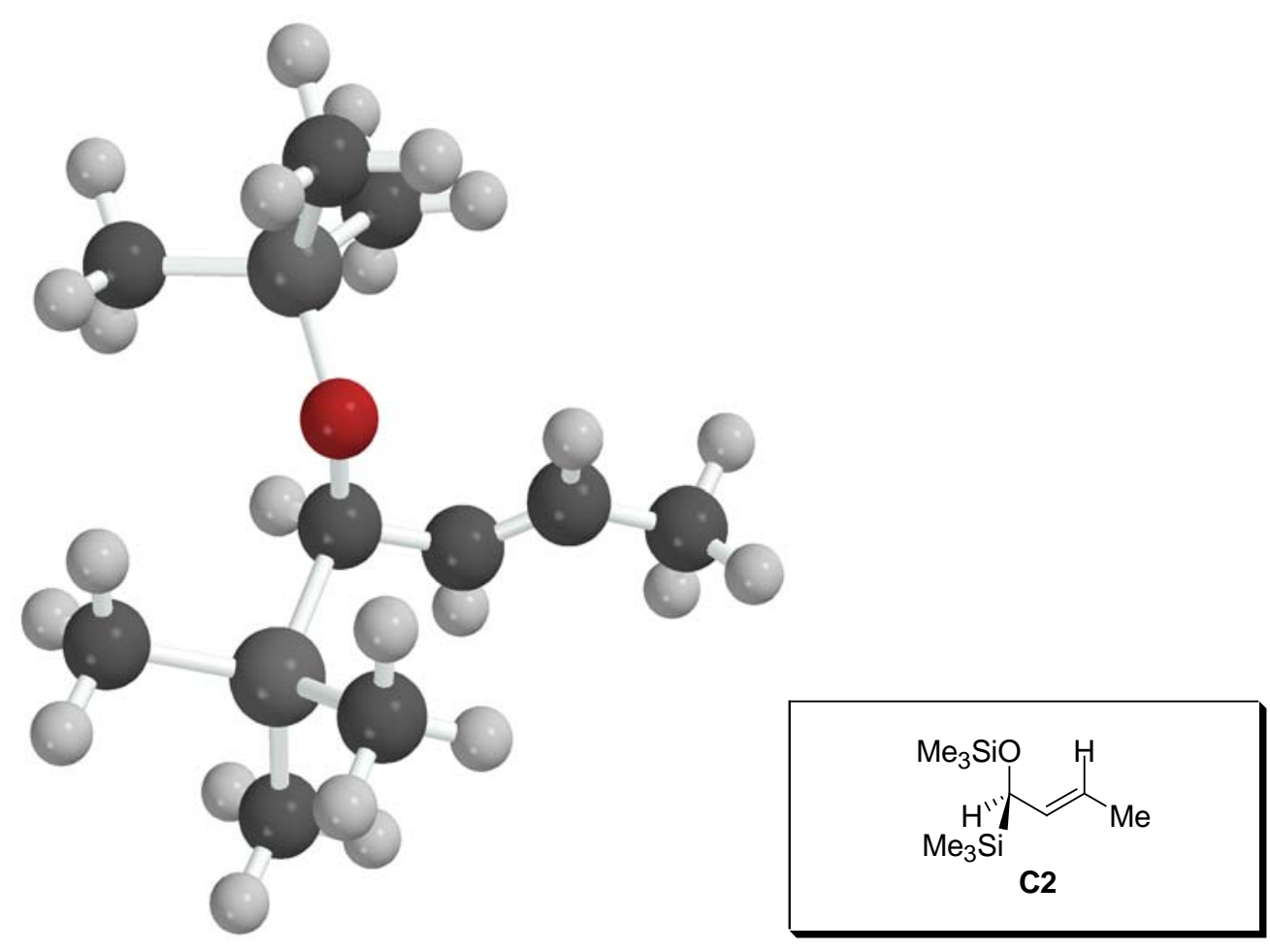

MacSPARTAN PRO Semi-Empirical Program: (PowerPC)

Release 1.0.4

Job run on machine : Woerpel G4(G4/466)

\section{CONF1.1}

Memory Used: $\quad 1.649 \mathrm{Mb}$

Reason for exit: Successful completion

Semi-Empirical Program CPU Time : 000:00:01.0

Semi-Empirical Program Wall Time: 000:00:02.5

MacSPARTAN PRO Ab-Initio Program: (PowerPC)

Job run on machine : Woerpel G4(G4/466)

Release 1.0.4

Calculation started: Sat Jan 202007 11:47:05 A

\section{CONF1.1}

Run type: Geometry optimization

Model: RHF/6-31G*

Number of shells: 102

61 S shells

28 SP shells

$136 \mathrm{D}$ shells

Number of basis functions: 251

Number of electrons: 120

Number of heavy atoms: 13

Number of hydrogens: 24

Use of molecular symmetry disabled 
Molecular charge: 0

Spin multiplicity: 1

Memory model: direct $21.7 \mathrm{Mb}$

Point Group $=$ C1 Order $=1$ Nsymop $=1$

This system has 105 degrees of freedom

Hessian from MMFF94 calculation used.

Max. Max. Neg.

Cycle Energy Grad. Dist. Eigen

$\begin{array}{llll}1 & -1045.4272652 & 0.02440 & 0.05151\end{array}$

$\begin{array}{llll}2 & -1045.4304305 & 0.03320 & 0.02485\end{array}$

$\begin{array}{llll}3 & -1045.4326916 & 0.01296 & 0.05746\end{array}$

$\begin{array}{llll}4 & -1045.4339696 & 0.00466 & 0.04147\end{array}$

$\begin{array}{llll}5 & -1045.4343401 & 0.00135 & 0.03632\end{array}$

$\begin{array}{llll}6 & -1045.4345551 & 0.00143 & 0.03086\end{array}$

$\begin{array}{llll}7 & -1045.4347140 & 0.00088 & 0.02369\end{array}$

$\begin{array}{lllll}8 & -1045.4348156 & 0.00078 & 0.02129\end{array}$

$\begin{array}{llll}9 & -1045.4348890 & 0.00052 & 0.02092\end{array}$

$\begin{array}{lllll}10 & -1045.4349300 & 0.00060 & 0.02064\end{array}$

$\begin{array}{llll}11 & -1045.4349597 & 0.00044 & 0.01861\end{array}$

$\begin{array}{llll}12 & -1045.4349733 & 0.00074 & 0.02279\end{array}$

$\begin{array}{llll}13 & -1045.4349788 & 0.00075 & 0.01117\end{array}$

$\begin{array}{llll}14 & -1045.4349773 & 0.00093 & 0.01345\end{array}$

$\begin{array}{lllll}15 & -1045.4349869 & 0.00061 & 0.00186\end{array}$

$\begin{array}{llll}16 & -1045.4349875 & 0.00074 & 0.00107\end{array}$

$\begin{array}{llll}17 & -1045.4349891 & 0.00034 & 0.00043\end{array}$

$\begin{array}{lllll}18 & -1045.4349912 & 0.00042 & 0.00164\end{array}$

$\begin{array}{llll}19 & -1045.4349918 & 0.00029 & 0.00172\end{array}$

$\begin{array}{llll}20 & -1045.4349916 & 0.00029 & 0.00059\end{array}$

$\begin{array}{llll}21 & -1045.4349918 & 0.00016 & 0.00112\end{array}$

\section{CONF1.1}

$\mathrm{E}(\mathrm{HF})=-1045.4349918$ a.u.

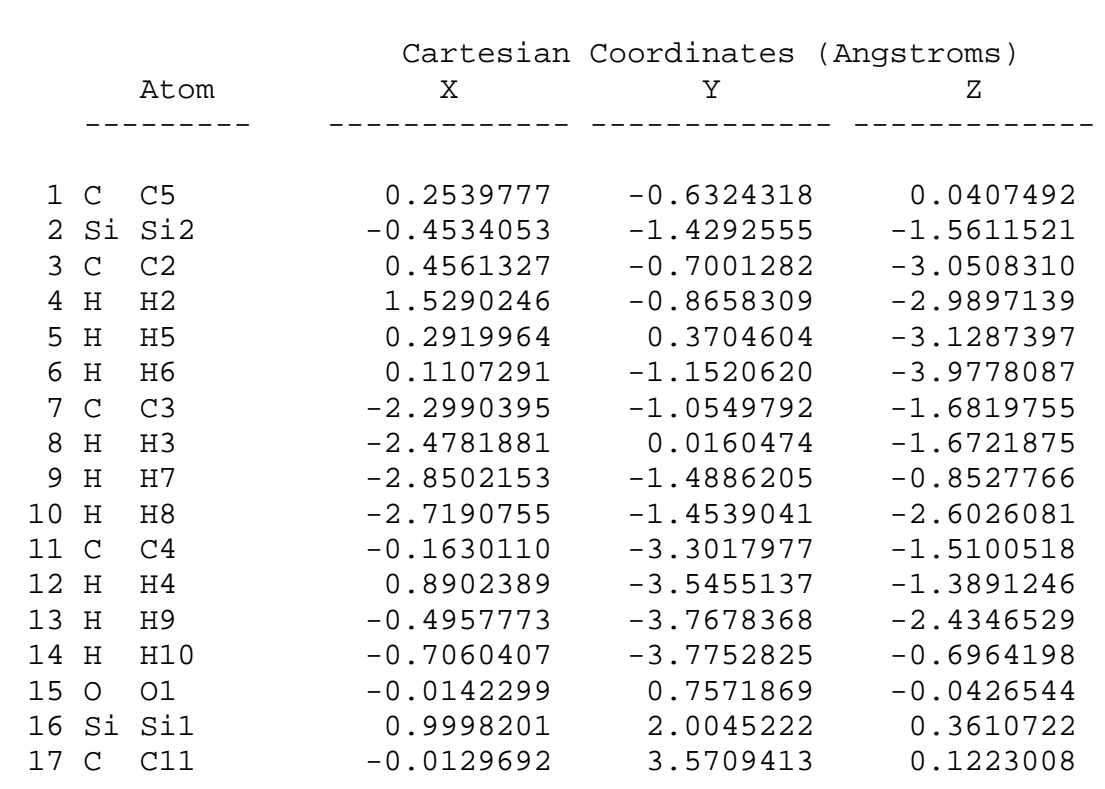




\begin{tabular}{|c|c|c|c|c|c|}
\hline 18 & $\mathrm{H}$ & $\mathrm{H} 1$ & $-\odot .8854178$ & 3.5783047 & 0.7703441 \\
\hline 19 & $\mathrm{H}$ & H25 & -0.3659289 & 3.6583262 & -0.9020368 \\
\hline 20 & $\mathrm{H}$ & $\mathrm{H} 26$ & $\odot .5713775$ & 4.4603586 & ๑. 3470657 \\
\hline 21 & C & $\mathrm{Cg}$ & 1.5967772 & 1.8620344 & 2.1458577 \\
\hline 22 & $\mathrm{H}$ & $\mathrm{H} 19$ & ๑. 7713859 & 1.9051099 & 2.8506226 \\
\hline 23 & $\mathrm{H}$ & $\mathrm{H} 21$ & 2.2787069 & 2.6750767 & 2.3863489 \\
\hline 24 & $\mathrm{H}$ & $\mathrm{H} 22$ & 2.1279411 & $\odot .9306062$ & 2.3251253 \\
\hline 25 & C & C10 & 2.5032994 & 2.0143129 & $-\odot .7824328$ \\
\hline 26 & $\mathrm{H}$ & $\mathrm{H} 20$ & 3.1611926 & 2.8482400 & -0.5470586 \\
\hline 27 & $\mathrm{H}$ & $\mathrm{H} 23$ & 2.2111902 & 2.1123024 & -1.8247053 \\
\hline 28 & $\mathrm{H}$ & $\mathrm{H} 24$ & 3.0947175 & 1.1060360 & $-\odot .6924391$ \\
\hline 29 & $\mathrm{H}$ & $\mathrm{H} 11$ & 1.3324597 & -0.8044687 & ๑. 0560609 \\
\hline 30 & C & $\mathrm{C} 6$ & -0.3195879 & -1.2489111 & 1.2895167 \\
\hline 31 & C & $\mathrm{C} 7$ & -1.2288760 & -0.7103261 & 2.0813936 \\
\hline 32 & $\mathrm{H}$ & $\mathrm{H} 13$ & -1.6010006 & ๑. 2733770 & 1.8504030 \\
\hline 33 & $\mathrm{C}$ & C12 & -1.7953694 & -1.3690497 & 3.3086372 \\
\hline 34 & $\mathrm{H}$ & H16 & -1.5971093 & -0.7748340 & 4.1973728 \\
\hline 35 & $\mathrm{H}$ & $\mathrm{H} 27$ & -1.3728411 & -2.3560224 & 3.4640821 \\
\hline 36 & $\mathrm{H}$ & $\mathrm{H} 28$ & -2.8750646 & -1.4728545 & 3. 2325149 \\
\hline 37 & $\mathrm{H}$ & $\mathrm{H} 14$ & ๑. . 0521802 & -2.2391338 & 1.5099016 \\
\hline
\end{tabular}

** Archive file written **

Memory Used: $21.985 \mathrm{Mb}$

Reason for exit: Successful completion

Ab-Initio Program CPU Time : 008:20:59.5

Ab-Initio Program Wall Time: 011:30:23.8

MacSPARTAN PRO Properties Program: (PowerPC)

Release 1.0.4

Reason for exit: Successful completion

Properties Program CPU Time : 000:00:03.2

Properties Program Wall Time: 000:00:04.3 


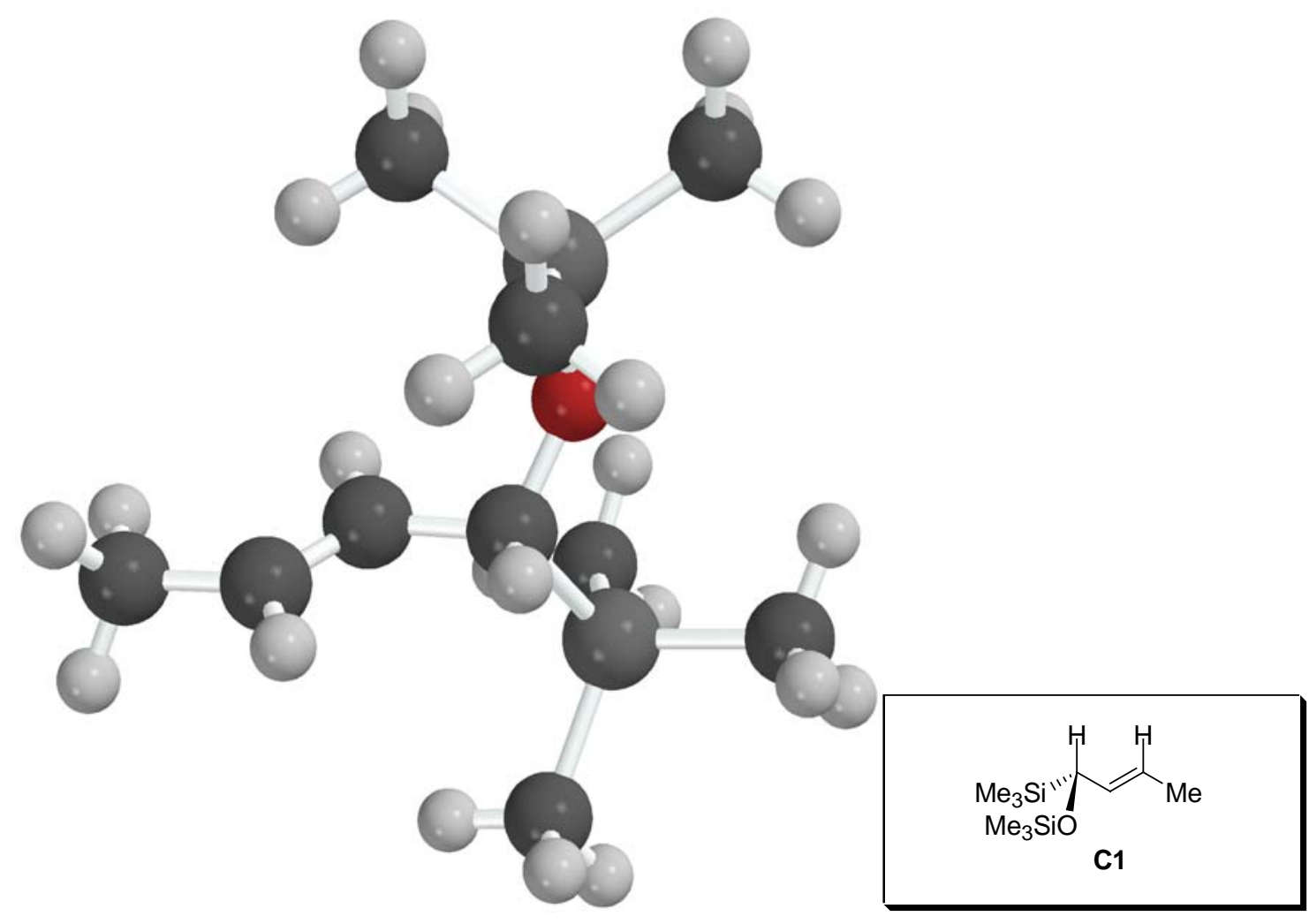

MacSPARTAN PRO Semi-Empirical Program: (PowerPC) Job run on machine : Woerpel G4(G4/466)

Release 1.0.4

CONF2.1

Memory Used: $\quad 1.649 \mathrm{Mb}$

Reason for exit: Successful completion

Semi-Empirical Program CPU Time : 000:00:01.0

Semi-Empirical Program Wall Time: 000:00:04.0

MacSPARTAN PRO Ab-Initio Program: (PowerPC)

Job run on machine : Woerpel G4(G4/466)

Release 1.0.4

Calculation started: Thu Jan 182007 4:41:09 PM

\section{CONF2.1}

Run type: Geometry optimization

Model: RHF/6-31G*

Number of shells: 102

61 S shells

28 SP shells

13 6D shells

Number of basis functions: 251

Number of electrons: $\quad 120$

Number of heavy atoms: 13

Number of hydrogens: 24

Use of molecular symmetry disabled

Molecular charge: 0 
Spin multiplicity: 1

Memory model: direct $21.7 \mathrm{Mb}$

Point Group $=$ C 1 Order $=1$ Nsymop $=1$

This system has 105 degrees of freedom

Hessian from MMFF94 calculation used.

Cycle Energy Grad. Dist. Eigen

$\begin{array}{llll}1 & -1045.4268540 & 0.02238 & 0.04451\end{array}$

$\begin{array}{llll}2 & -1045.4299758 & 0.03508 & 0.02278\end{array}$

$\begin{array}{llll}3 & -1045.4325749 & 0.01024 & 0.03823\end{array}$

$\begin{array}{llll}4 & -1045.4338284 & 0.00402 & 0.03313\end{array}$

$\begin{array}{llll}5 & -1045.4342551 & 0.00151 & 0.03872\end{array}$

$\begin{array}{llll}6 & -1045.4345305 & 0.00199 & 0.02932\end{array}$

$\begin{array}{llll}7 & -1045.4347233 & 0.00152 & 0.03438\end{array}$

$\begin{array}{llll}8 & -1045.4348524 & 0.00084 & 0.02185\end{array}$

$\begin{array}{llll}9 & -1045.4349481 & 0.00137 & 0.02293\end{array}$

$\begin{array}{lllll}10 & -1045.4350047 & 0.00128 & 0.02117\end{array}$

$\begin{array}{llll}11 & -1045.4350529 & 0.00095 & 0.02596\end{array}$

$\begin{array}{llll}12 & -1045.4350902 & 0.00090 & 0.02825\end{array}$

$\begin{array}{llll}13 & -1045.4351196 & 0.00074 & 0.02842\end{array}$

$\begin{array}{llll}14 & -1045.4351514 & 0.00099 & 0.02600\end{array}$

$\begin{array}{llll}15 & -1045.4351665 & 0.00206 & 0.02982\end{array}$

$\begin{array}{llll}16 & -1045.4351939 & 0.00125 & 0.02524\end{array}$

$\begin{array}{llll}17 & -1045.4351937 & 0.00170 & 0.02446\end{array}$

$\begin{array}{llll}18 & -1045.4352307 & 0.00091 & 0.01296\end{array}$

$\begin{array}{lllll}19 & -1045.4352254 & 0.00200 & 0.01191\end{array}$

$\begin{array}{llll}20 & -1045.4352405 & 0.00167 & 0.02160\end{array}$

$\begin{array}{llll}21 & -1045.4352133 & 0.00334 & 0.00614\end{array}$

$\begin{array}{lllll}22 & -1045.4352715 & 0.00109 & 0.01288\end{array}$

$\begin{array}{lllll}23 & -1045.4352460 & 0.00198 & 0.00742\end{array}$

$\begin{array}{lllll}24 & -1045.4352811 & 0.00101 & 0.01051\end{array}$

$\begin{array}{llll}25 & -1045.4352486 & 0.00269 & 0.02187\end{array}$

$\begin{array}{llll}26 & -1045.4352966 & 0.00070 & 0.02020\end{array}$

$\begin{array}{llll}27 & -1045.4352628 & 0.00136 & 0.02227\end{array}$

$\begin{array}{lllll}28 & -1045.4353046 & 0.00104 & 0.00804\end{array}$

$\begin{array}{lllll}29 & -1045.4352774 & 0.00203 & 0.01662\end{array}$

$\begin{array}{lllll}30 & -1045.4353184 & 0.00045 & 0.01732\end{array}$

$\begin{array}{llll}31 & -1045.4353113 & 0.00121 & 0.00901\end{array}$

$\begin{array}{lllll}32 & -1045.4353267 & 0.00071 & 0.01129\end{array}$

$\begin{array}{llll}33 & -1045.4353216 & 0.00100 & 0.01308\end{array}$

$\begin{array}{llll}34 & -1045.4353441 & 0.00058 & 0.02519\end{array}$

$\begin{array}{lllll}35 & -1045.4353407 & 0.00104 & 0.00168\end{array}$

$\begin{array}{llll}36 & -1045.4353599 & 0.00055 & 0.00324\end{array}$

$\begin{array}{lllll}37 & -1045.4353661 & 0.00044 & 0.00603\end{array}$

$\begin{array}{lllll}38 & -1045.4353748 & 0.00039 & 0.01037\end{array}$

$\begin{array}{llll}39 & -1045.4353844 & 0.00032 & 0.01785\end{array}$

$\begin{array}{llll}40 & -1045.4353985 & 0.00027 & 0.03241\end{array}$

$\begin{array}{lllll}41 & -1045.4354151 & 0.00045 & 0.03466\end{array}$

$\begin{array}{lllll}42 & -1045.4354324 & 0.00101 & 0.03071\end{array}$

$\begin{array}{lllll}43 & -1045.4354477 & 0.00066 & 0.02106\end{array}$

$\begin{array}{llll}44 & -1045.4354579 & 0.00127 & 0.03566\end{array}$

$\begin{array}{llll}45 & -1045.4354730 & 0.00082 & 0.00898\end{array}$

$\begin{array}{llll}46 & -1045.4354836 & 0.00061 & 0.01331\end{array}$

$\begin{array}{llll}47 & -1045.4354940 & 0.00087 & 0.00653\end{array}$

$\begin{array}{lllll}48 & -1045.4354696 & 0.00266 & 0.01260\end{array}$ 


$\begin{array}{llll}49 & -1045.4355114 & 0.00084 & 0.02013 \\ 50 & -1045.4355190 & 0.00100 & 0.01829 \\ 51 & -1045.4355331 & 0.00092 & 0.00412 \\ 52 & -1045.4355467 & 0.00099 & 0.00698 \\ 53 & -1045.4355596 & 0.00079 & 0.01334 \\ 54 & -1045.4355771 & 0.00040 & 0.02092 \\ 55 & -1045.4355916 & 0.00097 & 0.02501 \\ 56 & -1045.4356067 & 0.00108 & 0.02995 \\ 57 & -1045.4356233 & 0.00167 & 0.00980 \\ 58 & -1045.4356411 & 0.00070 & 0.01569 \\ 59 & -1045.4356584 & 0.00065 & 0.02832 \\ 60 & -1045.4356784 & 0.00077 & 0.02955 \\ 61 & -1045.4356948 & 0.00097 & 0.02791 \\ 62 & -1045.4357090 & 0.00103 & 0.00749 \\ 63 & -1045.4357242 & 0.00070 & 0.01633 \\ 64 & -1045.4357368 & 0.00064 & 0.01679 \\ 65 & -1045.4357424 & 0.00108 & 0.03129 \\ 66 & -1045.4357518 & 0.00138 & 0.00473 \\ 67 & -1045.4357446 & 0.00128 & 0.00575 \\ 68 & -1045.4357668 & 0.00080 & 0.00516 \\ 69 & -1045.4357655 & 0.00133 & 0.00721 \\ 70 & -1045.4357769 & 0.00054 & 0.00119 \\ 71 & -1045.4357806 & 0.00047 & 0.00223 \\ 72 & -1045.4357838 & 0.00037 & 0.00394 \\ 73 & -1045.4357882 & 0.00033 & 0.00745 \\ 74 & -1045.4357911 & 0.00047 & 0.01105 \\ 75 & -1045.4357949 & 0.00048 & 0.00776 \\ 76 & -1045.4357969 & 0.00032 & 0.00203 \\ 77 & -1045.4357993 & 0.00033 & 0.00196 \\ 78 & -1045.4358000 & 0.00052 & 0.00086 \\ 79 & -1045.4358016 & 0.00034 & 0.00175 \\ 80 & -1045.4358019 & 0.00038 & 0.00017 \\ 81 & -1045.4358018 & 0.00026 & 0.00029\end{array}$

\section{CONF2.1}

$\mathrm{E}(\mathrm{HF})=-1045.4358018$ a.u.

** Archive file written **

\begin{tabular}{|c|c|c|c|c|c|}
\hline & & & Cartesian & Coordinates & igstroms ) \\
\hline & & Atom & X & $\mathrm{Y}$ & Z \\
\hline & -- & - - - - - - & - - - - - - - - & - - - - - - - - - & ----- \\
\hline 1 & C & C5 & $\odot .1162693$ & -0.2194497 & $\odot .4498138$ \\
\hline 2 & $\mathrm{Si}$ & $\mathrm{Si2}$ & $-\odot .9592634$ & -1.6095313 & 1.2224214 \\
\hline 3 & $\mathrm{C}$ & $\mathrm{C} 2$ & -0.4829797 & -3.2466103 & 0.4063679 \\
\hline 4 & $\mathrm{H}$ & $\mathrm{H} 2$ & $\odot .5679915$ & -3.4790821 & $\odot .5607372$ \\
\hline 5 & $\mathrm{H}$ & H5 & -0.6592082 & -3.2145025 & -0.6644721 \\
\hline 6 & $\mathrm{H}$ & $\mathrm{H} 6$ & -1.0611143 & -4.0727403 & ๑. 8138465 \\
\hline 7 & C & C3 & -2.7874065 & -1.2386815 & $\odot .9175614$ \\
\hline 8 & $\mathrm{H}$ & H3 & -2.9857135 & -1.1064731 & $-\odot .1422986$ \\
\hline 9 & $\mathrm{H}$ & $\mathrm{H} 7$ & -3.1091708 & $-\odot .3358589$ & 1.4303383 \\
\hline 10 & $\mathrm{H}$ & H8 & -3.4150028 & -2.0533027 & 1.2713749 \\
\hline 11 & C & $\mathrm{C} 4$ & -0.6114767 & -1.7007663 & 3. 0817608 \\
\hline 12 & $\mathrm{H}$ & $\mathrm{H} 4$ & 0.4379818 & -1.9034165 & 3. 2842191 \\
\hline
\end{tabular}




$\begin{array}{rllrrr}13 & \mathrm{H} & \mathrm{H} 9 & -1.1886666 & -2.4978291 & 3.5451649 \\ 14 & \mathrm{H} & \mathrm{H} 10 & -0.8689512 & -0.7740369 & 3.5868748 \\ 15 & \text { O } & \text { 01 } & -0.1134123 & -0.2787405 & -0.9533188 \\ 16 & \mathrm{Si} & \mathrm{S} 11 & 0.7631556 & -.4012460 & -2.1845235 \\ 17 & \mathrm{C} & \mathrm{C} 11 & 0.3724337 & -0.6291699 & -3.7093902 \\ 18 & \mathrm{H} & \mathrm{H} 1 & -0.6921407 & -0.6143919 & -3.9278258 \\ 19 & \mathrm{H} & \mathrm{H} 25 & 0.6625902 & -1.6672890 & -3.5690132 \\ 20 & \mathrm{H} & \mathrm{H} 26 & 0.8922951 & -0.2553018 & -4.5887439 \\ 21 & \mathrm{C} & \mathrm{C} 9 & 0.2468574 & 2.1912444 & -2.4837967 \\ 22 & \mathrm{H} & \mathrm{H} 19 & -0.8209264 & 2.2638771 & -2.6772482 \\ 23 & \mathrm{H} & \mathrm{H} 21 & 0.7633774 & 2.6059508 & -3.3473371 \\ 24 & \mathrm{H} & \mathrm{H} 22 & 0.4709482 & 2.8266724 & -1.6316891 \\ 25 & \mathrm{C} & \mathrm{C} 10 & 2.6089000 & 0.3329229 & -1.7941690 \\ 26 & \mathrm{H} & \mathrm{H} 20 & 3.1888304 & 0.7017680 & -2.6372943 \\ 27 & \mathrm{H} & \mathrm{H} 23 & 2.9402911 & -0.6823505 & -1.5916373 \\ 28 & \mathrm{H} & \mathrm{H} 24 & 2.8687826 & 0.9433660 & -0.9334590 \\ 29 & \mathrm{H} & \mathrm{H} 11 & 1.1614488 & -0.4488745 & 0.6551767 \\ 30 & \mathrm{C} & \mathrm{C} 6 & -0.1865330 & 1.1507093 & 0.9927355 \\ 31 & \mathrm{C} & \mathrm{C} 7 & 0.5992505 & 1.8386328 & 1.8035949 \\ 32 & \mathrm{H} & \mathrm{H} 12 & 1.5540261 & 1.4199581 & 2.0861806 \\ 33 & \mathrm{C} & \mathrm{C} 8 & 0.2745248 & 3.1837402 & 2.3907389 \\ 34 & \mathrm{H} & \mathrm{H} 14 & 1.0247827 & 3.9201731 & 2.1143376 \\ 35 & \mathrm{H} & \mathrm{H} 15 & 0.2566277 & 3.1412207 & 3.4769099 \\ 36 & \mathrm{H} & \mathrm{H} 16 & -0.6914521 & 3.5417745 & 2.0516977 \\ 37 & \mathrm{H} & \mathrm{H} 13 & -1.1377639 & 1.5651433 & 0.6943637\end{array}$

Point Group $=\mathrm{C} 1$ order $=1$ Nsymop $=1$

Memory Used: $21.985 \mathrm{Mb}$

Reason for exit: Successful completion Ab-Initio Program CPU Time : 031:08:15.6 Ab-Initio Program Wall Time: 043:11:58.0

MacSPARTAN PRO Properties Program: (PowerPC) Reason for exit: Successful completion Properties Program CPU Time : 000:00:03.1 Properties Program Wall Time: 000:00:04.6 


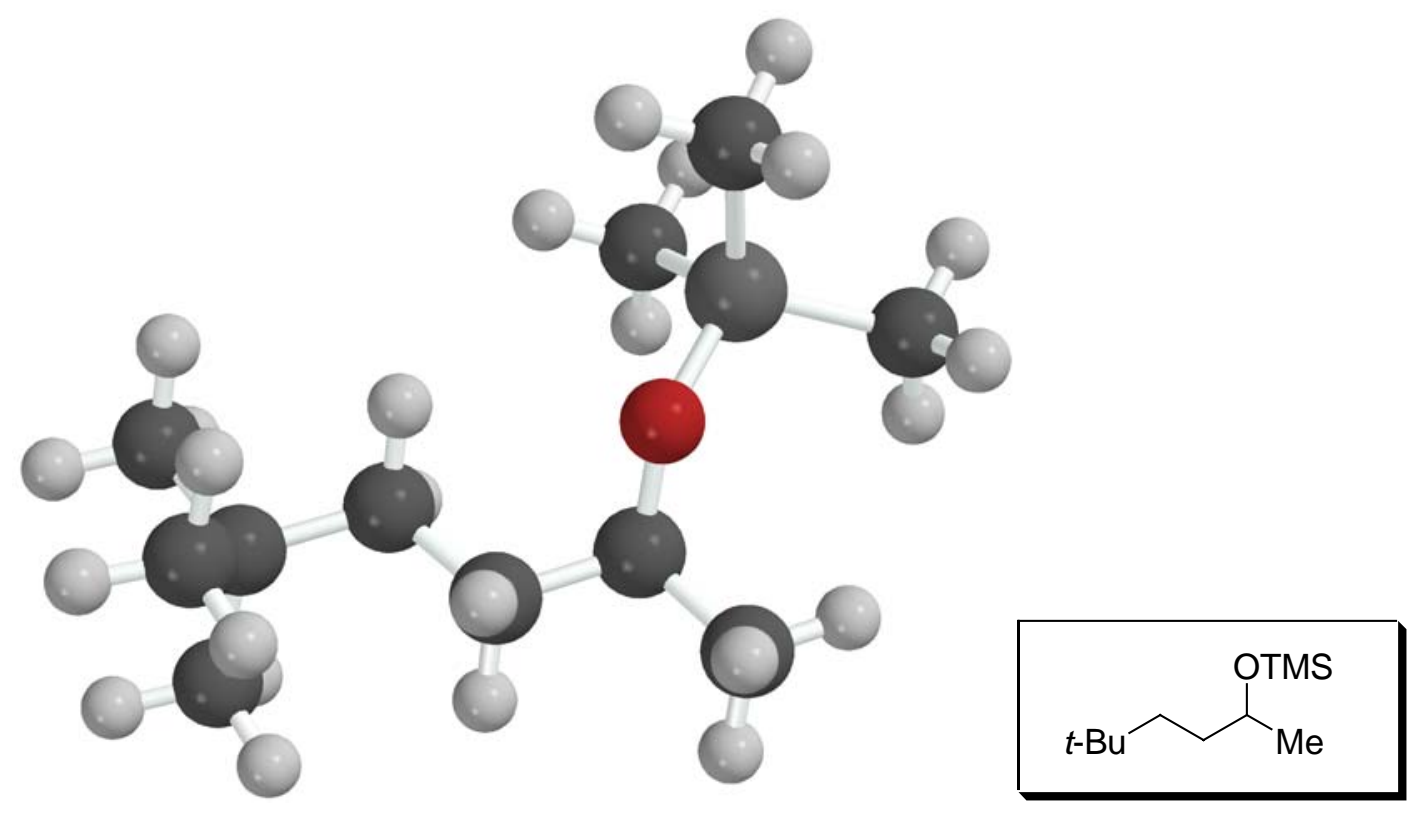

MacSPARTAN PRO Ab-Initio Program: (PowerPC) Job run on machine : Woerpel G4(G4/466)

Release 1.0.4

Calculation started: Fri May 112007 12:15:34 P

\section{TONYA1.1}

Run type: Geometry optimization

Model: RHF/6-31G*

Number of shells: 105

$65 \mathrm{~S}$ shells

27 SP shells

13 6D shells

Number of basis functions: 251

Number of electrons: 114

Number of heavy atoms: 13

Number of hydrogens: 26

Use of molecular symmetry disabled

Molecular charge: 0

Spin multiplicity: 1

Memory model: direct $21.6 \mathrm{Mb}$

Point Group = C1 Order $=1$ Nsymop $=1$

This system has 111 degrees of freedom

Hessian from AM1 calculation used.

Max. Max. Neg.

Cycle Energy Grad. Dist. Eigen

$\begin{array}{llll}1 & -795.5087891 & 0.05958 & 0.02510\end{array}$

$\begin{array}{lllll}2 & -795.5343267 & 0.02630 & 0.02469\end{array}$

$\begin{array}{lllll}3 & -795.5424547 & 0.00788 & 0.03222\end{array}$

$\begin{array}{lllll}4 & -795.5442376 & 0.00539 & 0.02844\end{array}$

$\begin{array}{lllll}5 & -795.5452036 & 0.00343 & 0.02781\end{array}$

$\begin{array}{lllll}6 & -795.5457396 & 0.00166 & 0.02885\end{array}$

$\begin{array}{lllll}7 & -795.5460919 & 0.00154 & 0.03794\end{array}$ 


$\begin{array}{cccc}8 & -795.5463889 & 0.00181 & 0.04443 \\ 9 & -795.5466392 & 0.00305 & 0.03951 \\ 10 & -795.5468218 & 0.00531 & 0.02846 \\ 11 & -795.5469906 & 0.00471 & 0.01775 \\ 12 & -795.5472398 & 0.00425 & 0.02879 \\ 13 & -795.5475275 & 0.00328 & 0.02795 \\ 14 & -795.5478392 & 0.00503 & 0.03732 \\ 15 & -795.5480446 & 0.00441 & 0.02993 \\ 16 & -795.5482656 & 0.00735 & 0.02365 \\ 17 & -795.5484989 & 0.00394 & 0.03955 \\ 18 & -795.5486832 & 0.00499 & 0.02256 \\ 19 & -795.5488445 & 0.00433 & 0.02008 \\ 20 & -795.5489715 & 0.00315 & 0.02415 \\ 21 & -795.5491303 & 0.00343 & 0.02039 \\ 22 & -795.5492771 & 0.00295 & 0.03187 \\ 23 & -795.5493829 & 0.00475 & 0.01894 \\ 24 & -795.5493351 & 0.00496 & 0.00594 \\ 25 & -795.5496108 & 0.00225 & 0.01201 \\ 26 & -795.5496687 & 0.00213 & 0.01719 \\ 27 & -795.5497393 & 0.00202 & 0.02226 \\ 28 & -795.5498581 & 0.00150 & 0.03349 \\ 29 & -795.5499390 & 0.00240 & 0.02824 \\ 30 & -795.5500233 & 0.00172 & 0.02348 \\ 31 & -795.5500918 & 0.00100 & 0.03258 \\ 32 & -795.5501601 & 0.00150 & 0.03461 \\ 33 & -795.5502249 & 0.00128 & 0.03134 \\ 34 & -795.5502761 & 0.00150 & 0.02772 \\ 35 & -795.5503272 & 0.00137 & 0.02496 \\ 36 & -795.5503582 & 0.00216 & 0.02647 \\ 37 & -795.5504155 & 0.00162 & 0.02562 \\ 38 & -795.5504572 & 0.00090 & 0.02200 \\ 39 & -795.5504839 & 0.00165 & 0.01151 \\ 40 & -795.5504879 & 0.00141 & 0.02088 \\ 41 & -795.5505067 & 0.00100 & 0.00489 \\ 42 & -795.5505154 & 0.00164 & 0.00549 \\ 43 & -795.5505266 & 0.00107 & 0.00454 \\ 44 & -795.5505379 & 0.00070 & 0.00958 \\ 45 & -795.5505481 & 0.00040 & 0.01134 \\ 46 & -795.5505589 & 0.00052 & 0.00320 \\ 47 & -795.5505646 & 0.00059 & 0.00573 \\ 48 & -795.5505710 & 0.00048 & 0.00389 \\ 49 & -795.5505756 & 0.00068 & 0.00248 \\ 50 & -795.5505796 & 0.00062 & 0.00376 \\ 51 & -795.5505868 & 0.00063 & 0.00545 \\ 52 & -795.5505950 & 0.00057 & 0.00866 \\ 53 & -795.5506057 & 0.00045 & 0.01490 \\ 54 & -795.5506205 & 0.00053 & 0.02895 \\ 55 & -795.5506403 & 0.00061 & 0.02977 \\ 56 & -795.5506576 & 0.00062 & 0.02264 \\ 57 & -795.5506744 & 0.00091 & 0.03107 \\ 58 & -795.5506912 & 0.00106 & 0.00573 \\ 59 & -795.5507042 & 0.00079 & 0.00901 \\ 60 & -795.5507137 & 0.00063 & 0.00662 \\ 61 & -795.5507139 & 0.00081 & 0.01600 \\ 62 & -795.5507203 & 0.00079 & 0.00301 \\ 63 & -795.5507248 & 0.00030 & 0.00264\end{array}$




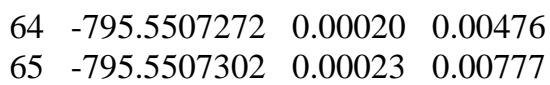

\section{TONYA1.1}

$\mathrm{E}(\mathrm{HF})=-795.5507302$ a.u.

** Archive file written **

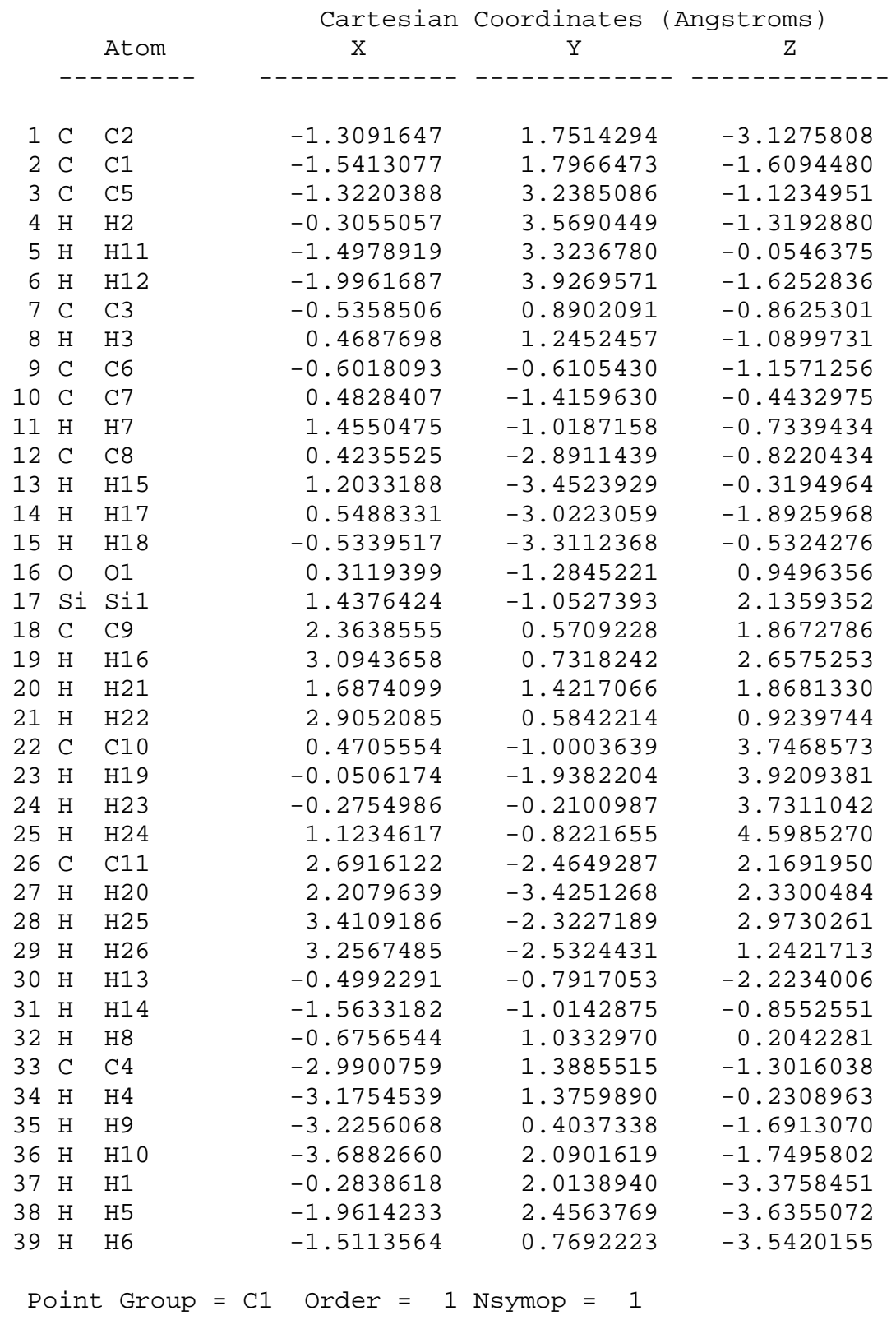

Memory Used: $21.913 \mathrm{Mb}$

Reason for exit: Successful completion Ab-Initio Program CPU Time : 024:14:45.7 Ab-Initio Program Wall Time: 034:13:49.4 
MacSPARTAN PRO Properties Program: (PowerPC)

Reason for exit: Successful completion

Release 1.0.4

Properties Program CPU Time : 000:00:03.1

Properties Program Wall Time: 000:00:04.5

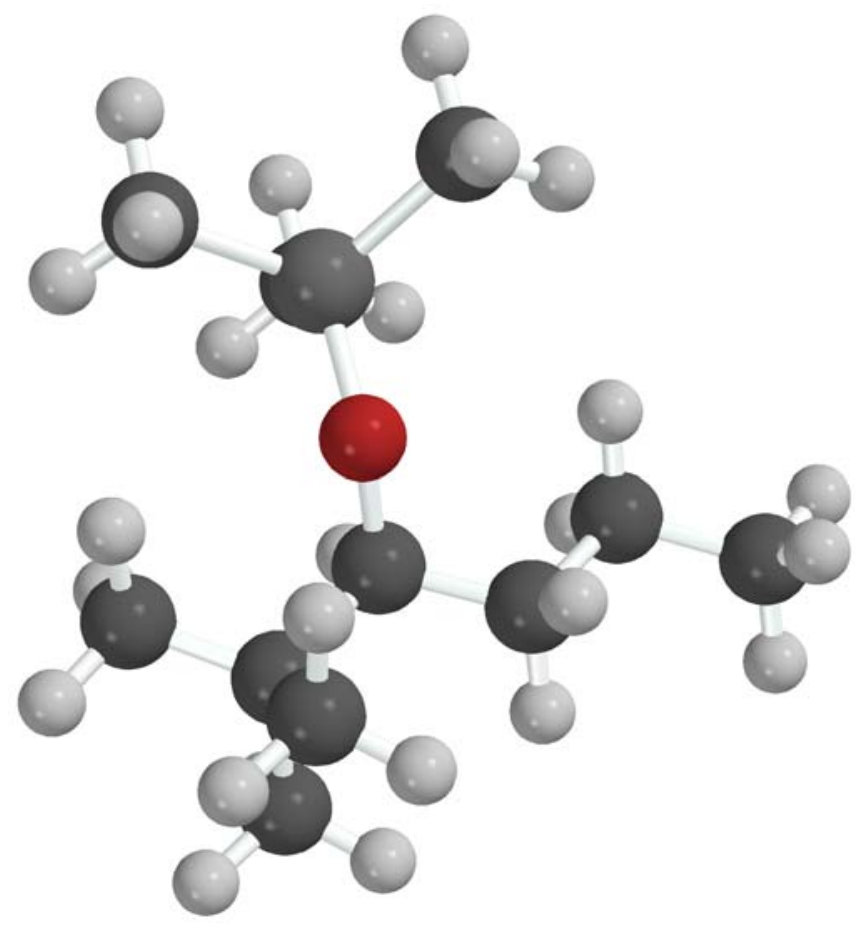

MacSPARTAN PRO Ab-Initio Program: (PowerPC)

Job run on machine : Woerpel G4(G4/466)

Calculation started: Sat May 122007 10:24:30 P

TONYA2.1

Run type: Geometry optimization

Model: RHF/6-31G*

Number of shells: 105

65 S shells

27 SP shells

13 6D shells

Number of basis functions: 251

Number of electrons: 114

Number of heavy atoms: 13

Number of hydrogens: $\quad 26$

Use of molecular symmetry disabled

Molecular charge: 0

Spin multiplicity: 1

Memory model: direct $21.6 \mathrm{Mb}$

Point Group = C1 Order $=1$ Nsymop $=1$

This system has 111 degrees of freedom

Hessian from AM1 calculation used.

Max. Max. Neg. 


$\begin{array}{cccc}\text { Cycle } & \text { Energy } & \text { Grad. } & \text { Dist. } \\ 1 & -795.5025917 & 0.06468 & 0.02742 \\ 2 & -795.5291649 & 0.03228 & 0.01838 \\ 3 & -795.5375186 & 0.00758 & 0.02588 \\ 4 & -795.5392710 & 0.00280 & 0.02604 \\ 5 & -795.5402442 & 0.00257 & 0.02541 \\ 6 & -795.5408921 & 0.00155 & 0.02391 \\ 7 & -795.5413553 & 0.00171 & 0.02172 \\ 8 & -795.5417191 & 0.00176 & 0.02444 \\ 9 & -795.5420086 & 0.00211 & 0.02381 \\ 10 & -795.5422347 & 0.00215 & 0.03857 \\ 11 & -795.5424078 & 0.00346 & 0.02436 \\ 12 & -795.5425910 & 0.00294 & 0.02553 \\ 13 & -795.5427654 & 0.00259 & 0.02790 \\ 14 & -795.5429300 & 0.00179 & 0.02790 \\ 15 & -795.5430848 & 0.00296 & 0.02182 \\ 16 & -795.5432226 & 0.00268 & 0.02467 \\ 17 & -795.5433597 & 0.00277 & 0.02917 \\ 18 & -795.5435318 & 0.00243 & 0.02889 \\ 19 & -795.5436965 & 0.00317 & 0.03101 \\ 20 & -795.5438765 & 0.00268 & 0.03025 \\ 21 & -795.5440468 & 0.00313 & 0.02921 \\ 22 & -795.5441825 & 0.00263 & 0.03078 \\ 23 & -795.5443149 & 0.00289 & 0.03007 \\ 24 & -795.5444314 & 0.00269 & 0.03156 \\ 25 & -795.5445764 & 0.00192 & 0.03912 \\ 26 & -795.5447010 & 0.00293 & 0.03243 \\ 27 & -795.5448054 & 0.00188 & 0.02992 \\ 28 & -795.5449148 & 0.00129 & 0.03347 \\ 29 & -795.5449919 & 0.00173 & 0.02501 \\ 30 & -795.5450428 & 0.00169 & 0.01583 \\ 31 & -795.5450855 & 0.00108 & 0.02650 \\ 32 & -795.5451292 & 0.00153 & 0.03923 \\ 33 & -795.5451209 & 0.00260 & 0.00481 \\ 34 & -795.5451489 & 0.00139 & 0.00755 \\ 35 & -795.5451596 & 0.00155 & 0.00186 \\ 36 & -795.5451772 & 0.00056 & 0.00339 \\ 37 & -795.5451838 & 0.00060 & 0.00597 \\ 38 & -795.5451924 & 0.00057 & 0.01045 \\ 39 & -795.5452067 & 0.00041 & 0.01979 \\ 40 & -795.5452206 & 0.00086 & 0.02288 \\ 41 & -795.5452291 & 0.00084 & 0.00053 \\ 42 & -795.5452394 & 0.00084 & 0.00153 \\ 43 & -795.5452499 & 0.00125 & 0.00292 \\ 44 & -795.5452606 & 0.00118 & 0.00514 \\ 45 & -795.5452738 & 0.00107 & 0.00824 \\ 46 & -795.5452900 & 0.00091 & 0.01207 \\ 47 & -795.5453109 & 0.00077 & 0.02188 \\ 48 & -795.5453273 & 0.00093 & 0.01104 \\ 49 & -795.5453458 & 0.00132 & 0.01197 \\ 50 & -795.5453738 & 0.00085 & 0.01118 \\ 51 & -795.5453894 & 0.00129 & 0.02202 \\ 52 & -795.5454039 & 0.00120 & 0.01350 \\ 53 & -795.5454214 & 0.00088 & 0.02160 \\ 54 & -795.5454328 & 0.00083 & 0.00148 \\ 55 & -795.5454450 & 0.00061 & 0.00266 \\ & & & \end{array}$




$\begin{array}{llll}56 & -795.5454531 & 0.00056 & 0.00453 \\ 57 & -795.5454638 & 0.00042 & 0.00825 \\ 58 & -795.5454753 & 0.00043 & 0.01500 \\ 59 & -795.5454886 & 0.00033 & 0.02599 \\ 60 & -795.5455002 & 0.00053 & 0.01440 \\ 61 & -795.5455082 & 0.00038 & 0.01205 \\ 62 & -795.5455129 & 0.00060 & 0.00481 \\ 63 & -795.5455152 & 0.00086 & 0.00859 \\ 64 & -795.5455218 & 0.00061 & 0.00742 \\ 65 & -795.5455281 & 0.00057 & 0.00364 \\ 66 & -795.5455357 & 0.00047 & 0.00577 \\ 67 & -795.5455450 & 0.00040 & 0.01054 \\ 68 & -795.5455534 & 0.00045 & 0.02439 \\ 69 & -795.5455625 & 0.00063 & 0.02596 \\ 70 & -795.5455699 & 0.00098 & 0.00517 \\ 71 & -795.5455681 & 0.00118 & 0.01027 \\ 72 & -795.5455960 & 0.00065 & 0.01738 \\ 73 & -795.5456095 & 0.00118 & 0.00558 \\ 74 & -795.5456223 & 0.00084 & 0.00263 \\ 75 & -795.5456374 & 0.00063 & 0.00446 \\ 76 & -795.5456478 & 0.00057 & 0.00714 \\ 77 & -795.5456612 & 0.00048 & 0.01620 \\ 78 & -795.5456728 & 0.00064 & 0.02173 \\ 79 & -795.5456868 & 0.00093 & 0.01252 \\ 80 & -795.5456986 & 0.00066 & 0.00157 \\ 81 & -795.5457036 & 0.00053 & 0.00154 \\ 82 & -795.5457091 & 0.00036 & 0.00221 \\ 83 & -795.5457140 & 0.00046 & 0.00432 \\ 84 & -795.5457183 & 0.00061 & 0.00878 \\ 85 & -795.5457244 & 0.00048 & 0.00972 \\ 86 & -795.5457282 & 0.00070 & 0.00958 \\ 87 & -795.5457327 & 0.00065 & 0.00136 \\ 88 & -795.5457340 & 0.00034 & 0.00303 \\ 89 & -795.5457370 & 0.00045 & 0.00034 \\ 90 & -795.5457389 & 0.00056 & 0.00050 \\ 91 & -795.5457409 & 0.00042 & 0.00110 \\ 92 & -795.5457429 & 0.00016 & 0.00211 \\ 93 & -795.5457440 & 0.00028 & 0.00085 \\ 94 & -795.5457398 & 0.00020 & 0.00024\end{array}$

TONYA2.1

$\mathrm{E}(\mathrm{HF})=-795.5457398$ a.u.

** Archive file written **

\begin{tabular}{|c|c|c|c|c|}
\hline & & Cartesian & Coordinates ( & (Angstroms) \\
\hline & Atom & $\mathrm{x}$ & $\mathrm{Y}$ & Z \\
\hline & - - - - - & - - - - - - - - - & $-\cdots$ & - - - \\
\hline C & $\mathrm{C} 5$ & -0.5254272 & ๑. 4326996 & $\odot .2129284$ \\
\hline C & $\mathrm{C} 1$ & -1.0294575 & $\odot .6669444$ & 1.6622078 \\
\hline C & $\mathrm{C} 2$ & 0.1791875 & $\odot .9149784$ & 2.5780839 \\
\hline $\mathrm{H}$ & $\mathrm{H} 2$ & 0.7973902 & 1.7292931 & 2. 2064782 \\
\hline
\end{tabular}




\begin{tabular}{|c|c|c|c|c|}
\hline 5 & H5 & 0.7975395 & ๑. .0322052 & 2.6660555 \\
\hline 6 & $\mathrm{H} 6$ & -0.1528372 & 1.1874929 & 3.5754305 \\
\hline 7 & C3 & -1.8086454 & $-\odot .5459634$ & 2.1901580 \\
\hline 8 & H3 & -1.2179413 & -1.4516122 & 2.1107867 \\
\hline 9 & $\mathrm{H} 7$ & -2.7366094 & -0.6981652 & 1.6486035 \\
\hline 10 & H8 & -2.0640455 & $-\odot .4017667$ & 3.2364666 \\
\hline 11 & C4 & -1.9231249 & 1.9187345 & 1.7138131 \\
\hline 12 & $\mathrm{H} 4$ & -1.4161277 & 2.7863781 & 1.2989078 \\
\hline 13 & $\mathrm{H} 9$ & -2.1765528 & 2.1517562 & 2.7433547 \\
\hline 14 & $\mathrm{H} 10$ & -2.8557067 & 1.7889798 & 1.1772093 \\
\hline 15 & 01 & ๑. 4098680 & -0.6239312 & ๑. 2000630 \\
\hline 16 & Si Si1 & 1.9796960 & -0.7834713 & -0.2823675 \\
\hline 17 & C $\quad$ C11 & 3.0579575 & -1.2085208 & 1.2058362 \\
\hline 18 & $\mathrm{H} 1$ & 4.0651709 & -1.4701705 & ๑.8879078 \\
\hline 19 & $\mathrm{H} 25$ & 2.6579979 & -2.0598657 & 1.7511545 \\
\hline 20 & $\mathrm{H} 26$ & 3.1418103 & -0.3810400 & 1.9044090 \\
\hline 21 & C9 & 2.0824532 & -2.2138299 & -1.5066435 \\
\hline 22 & H19 & 1.6643094 & -3.1213848 & -1.0784946 \\
\hline 23 & $\mathrm{H} 21$ & 3.1161526 & -2.4249148 & -1.7730695 \\
\hline 24 & $\mathrm{H} 22$ & 1.5439107 & -2.0104362 & -2.4275078 \\
\hline 25 & C10 & 2.6158599 & ๑. 8056033 & -1.0812696 \\
\hline 26 & $\mathrm{H} 20$ & 3.6477120 & 0.6710714 & -1.3987349 \\
\hline 27 & $\mathrm{H} 23$ & 2.6003570 & 1.6470358 & -0.3929334 \\
\hline 28 & $\mathrm{H} 24$ & 2.0420790 & 1.0881634 & -1.9599868 \\
\hline 29 & $\mathrm{H} 11$ & $-\odot .0297497$ & 1.3507096 & $-\odot .0978551$ \\
\hline 30 & C6 & -1.6265485 & 0.1131305 & -0.8037428 \\
\hline 31 & $\mathrm{C} 7$ & -1.1725043 & ๑. 2030602 & -2.2620449 \\
\hline 32 & $\mathrm{H} 12$ & -0.7966590 & 1.2045570 & -2.4634232 \\
\hline 33 & $\mathrm{C} 8$ & -2.2986873 & -0.1184081 & -3.2448947 \\
\hline 34 & H15 & -1.9554106 & $-\odot .0479906$ & -4.2724630 \\
\hline 35 & $\mathrm{H} 17$ & -3.1323260 & ๑. 5681852 & -3.1273079 \\
\hline 36 & H18 & -2.6762406 & -1.1251761 & -3.0907014 \\
\hline 37 & H16 & $-\odot .3481426$ & -0.4797584 & -2.4343663 \\
\hline 38 & $\mathrm{H} 13$ & -2.4606615 & ๑. 7931865 & -0.6669841 \\
\hline 39 & H14 & -1.9959820 & $-\odot .8877590$ & $-\odot .6050634$ \\
\hline
\end{tabular}

Point Group $=\mathrm{C} 1$ Order $=1$ Nsymop $=1$

Memory Used: $21.913 \mathrm{Mb}$

Reason for exit: Successful completion

Ab-Initio Program CPU Time : 037:35:35.0

Ab-Initio Program Wall Time: 053:35:02.9

MacSPARTAN PRO Properties Program: (PowerPC)

Release 1.0.4

Reason for exit: Successful completion

Properties Program CPU Time : 000:00:03.1

Properties Program Wall Time: 000:00:04.5 


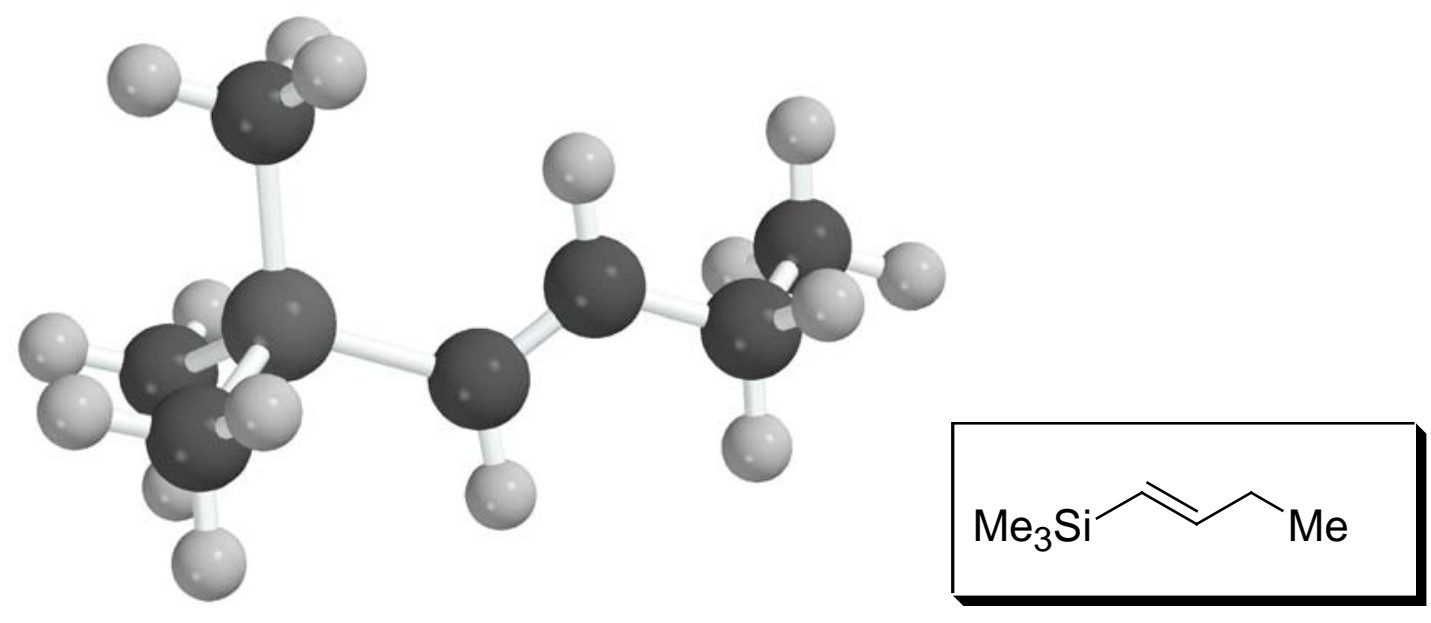

MacSPARTAN PRO Ab-Initio Program: (PowerPC)

Release 1.0.4

Job run on machine : Woerpel G4(G4/466)

Calculation started: Fri May 112007 3:14:38 AM

\section{TONYB1.1}

Run type: Geometry optimization

Model: RHF/6-31G*

Number of shells: 65

40 S shells

17 SP shells

8 6D shells

Number of basis functions: 156

Number of electrons: 72

Number of heavy atoms: 8

Number of hydrogens: 16

Use of molecular symmetry disabled

Molecular charge: 0

Spin multiplicity: 1

Memory model: direct $10.3 \mathrm{Mb}$

Point Group = C1 Order $=1$ Nsymop $=1$

This system has 66 degrees of freedom

Hessian from AM1 calculation used.

Max. Max. Neg.

Cycle Energy Grad. Dist. Eigen

$\begin{array}{cccc}1 & -563.3065371 & 0.04126 & 0.02880 \\ 2 & -563.3208591 & 0.01282 & 0.02195 \\ 3 & -563.3253392 & 0.00693 & 0.02479 \\ 4 & -563.3263387 & 0.00583 & 0.03647 \\ 5 & -563.3266671 & 0.00252 & 0.03309 \\ 6 & -563.3267480 & 0.00256 & 0.04324 \\ 7 & -563.3269048 & 0.00242 & 0.05394 \\ 8 & -563.3269570 & 0.00419 & 0.03591 \\ 9 & -563.3270535 & 0.00296 & 0.00992 \\ 10 & -563.3271305 & 0.00229 & 0.02938 \\ 11 & -563.3272103 & 0.00260 & 0.05206 \\ 12 & -563.3272959 & 0.00387 & 0.02939\end{array}$




$$
\begin{array}{llll}
13 & -563.3273887 & 0.00316 & 0.00690 \\
14 & -563.3274666 & 0.00215 & 0.01487 \\
15 & -563.3275626 & 0.00175 & 0.03219 \\
16 & -563.3276727 & 0.00144 & 0.03256 \\
17 & -563.3277386 & 0.00304 & 0.03928 \\
18 & -563.3277479 & 0.00393 & 0.00767 \\
19 & -563.3279079 & 0.00377 & 0.01233 \\
20 & -563.3279813 & 0.00280 & 0.02084 \\
21 & -563.3280799 & 0.00278 & 0.03805 \\
22 & -563.3282223 & 0.00114 & 0.03486 \\
23 & -563.3282987 & 0.00270 & 0.02670 \\
24 & -563.3283757 & 0.00147 & 0.03132 \\
25 & -563.3284371 & 0.00186 & 0.01569 \\
26 & -563.3284510 & 0.00290 & 0.01742 \\
27 & -563.3284879 & 0.00301 & 0.00838 \\
28 & -563.3284614 & 0.00391 & 0.00386 \\
29 & -563.3285274 & 0.00277 & 0.00972 \\
30 & -563.3285101 & 0.00386 & 0.00643 \\
31 & -563.3285702 & 0.00139 & 0.00067 \\
32 & -563.3285746 & 0.00112 & 0.00126 \\
33 & -563.3285849 & 0.00085 & 0.00221 \\
34 & -563.3286000 & 0.00050 & 0.00359 \\
35 & -563.3286124 & 0.00061 & 0.00655 \\
36 & -563.3286281 & 0.00086 & 0.01072 \\
37 & -563.3286438 & 0.00118 & 0.01837 \\
38 & -563.3286617 & 0.00176 & 0.00664 \\
39 & -563.3286855 & 0.00176 & 0.01172 \\
40 & -563.3287032 & 0.00085 & 0.00785 \\
41 & -563.3287174 & 0.00127 & 0.01684 \\
42 & -563.3287324 & 0.00079 & 0.00107 \\
43 & -563.3287491 & 0.00079 & 0.00228 \\
44 & -563.3287630 & 0.00100 & 0.00362 \\
45 & -563.3287833 & 0.00120 & 0.00639 \\
46 & -563.3288041 & 0.00113 & 0.00658 \\
47 & -563.3288289 & 0.00073 & 0.02116 \\
48 & -563.3288269 & 0.00120 & 0.00637 \\
49 & -563.3288378 & 0.00078 & 0.00021 \\
50 & -563.3288421 & 0.00069 & 0.00033 \\
51 & -563.3288464 & 0.00051 & 0.00050 \\
52 & -563.3288497 & 0.00051 & 0.00135 \\
53 & -563.3288534 & 0.00052 & 0.00205 \\
54 & -563.3288557 & 0.00060 & 0.00041 \\
55 & -563.3288583 & 0.00026 & 0.00060 \\
56 & -563.3288603 & 0.00034 & 0.00101 \\
57 & -563.3288626 & 0.00032 & 0.00110 \\
58 & -563.3288647 & 0.00033 & 0.00051 \\
59 & -563.3288665 & 0.00032 & 0.00031 \\
60 & -563.3288686 & 0.00026 & 0.00040 \\
61 & -563.3288705 & 0.00021 & 0.00011
\end{array}
$$

\section{TONYB1.1}

$\mathrm{E}(\mathrm{HF})=-563.3288705$ a.u. 


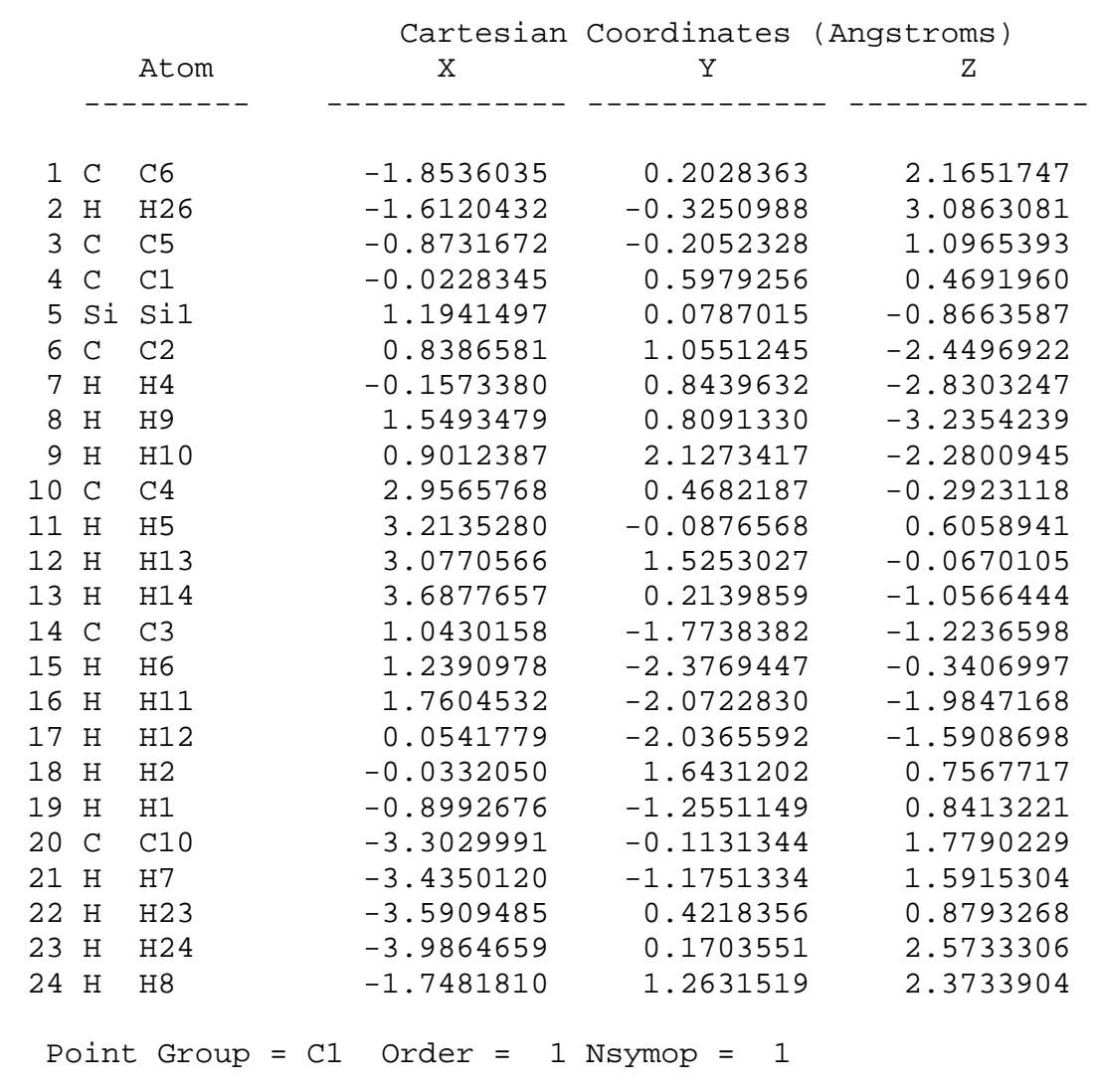

** Archive file written **

Memory Used: $10.462 \mathrm{Mb}$

Reason for exit: Successful completion

Ab-Initio Program CPU Time : 005:54:38.0

Ab-Initio Program Wall Time: 008:14:02.1

MacSPARTAN PRO Properties Program: (PowerPC)

Release 1.0.4

Reason for exit: Successful completion

Properties Program CPU Time : 000:00:01.0

Properties Program Wall Time: 000:00:02.0 


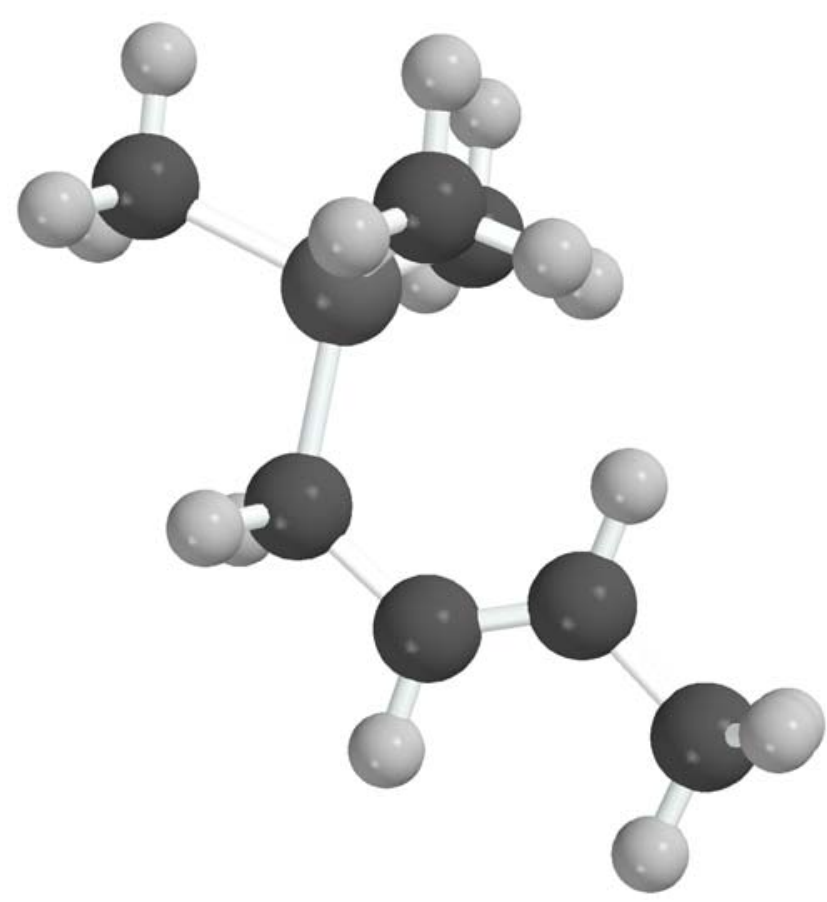

MacSPARTAN PRO Ab-Initio Program: (PowerPC)

Job run on machine : Woerpel G4(G4/466)

Calculation started: Fri May 112007 11:27:32 A

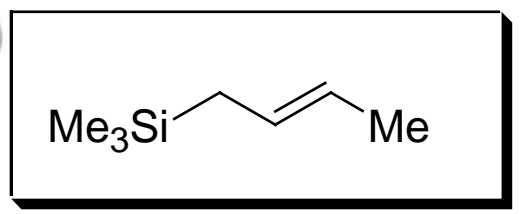

Release 1.0.4

\section{TONYB2.1}

Run type: Geometry optimization

Model: RHF/6-31G*

Number of shells: 65

40 S shells

17 SP shells

8 6D shells

Number of basis functions: 156

Number of electrons: $\quad 72$

Number of heavy atoms: 8

Number of hydrogens: 16

Use of molecular symmetry enabled

Molecular charge: 0

Spin multiplicity: 1

Memory model: direct $10.5 \mathrm{Mb}$

Point Group = CS Order $=1$ Nsymop $=2$

This system has 38 degrees of freedom

Hessian from AM1 calculation used.

Max. Max. Neg.

Cycle Energy Grad. Dist. Eigen

$\begin{array}{llll}1 & -563.3042448 & 0.03933 & 0.03335\end{array}$

$\begin{array}{lllll}2 & -563.3190603 & 0.01514 & 0.02578\end{array}$

$\begin{array}{lllll}3 & -563.3217273 & 0.00627 & 0.02933\end{array}$

$\begin{array}{lllll}4 & -563.3222325 & 0.00248 & 0.02988\end{array}$

$\begin{array}{lllll}5 & -563.3223157 & 0.00215 & 0.02792\end{array}$

$\begin{array}{llll}6 & -563.3223000 & 0.00241 & 0.00478\end{array}$ 
$\begin{array}{llll}7 & -563.3223671 & 0.00072 & 0.00132\end{array}$

$\begin{array}{llll}8 & -563.3223731 & 0.00036 & 0.00027\end{array}$

$9 \begin{array}{llll}9 & -563.3223752 & 0.00029 & 0.00021\end{array}$

\section{TONYB2.1}

$\mathrm{E}(\mathrm{HF})=-563.3223752$ a.u.

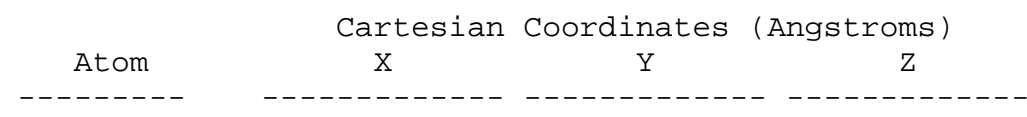

$\begin{array}{rll}1 & \mathrm{H} & \mathrm{H} 1 \\ 2 & \mathrm{C} & \mathrm{C} 1 \\ 3 & \mathrm{Si} & \mathrm{Si} 1 \\ 4 & \mathrm{C} & \mathrm{C} 2 \\ 5 & \mathrm{H} & \mathrm{H} 4 \\ 6 & \mathrm{H} & \mathrm{H} 9 \\ 7 & \mathrm{H} & \mathrm{H} 10 \\ 8 & \mathrm{C} & \mathrm{C} 4 \\ 9 & \mathrm{H} & \mathrm{H} 5 \\ 10 & \mathrm{H} & \mathrm{H} 13 \\ 11 & \mathrm{H} & \mathrm{H} 14 \\ 12 & \mathrm{C} & \mathrm{C} 3 \\ 13 & \mathrm{H} & \mathrm{H} 6 \\ 14 & \mathrm{H} & \mathrm{H} 11 \\ 15 & \mathrm{H} & \mathrm{H} 12 \\ 16 & \mathrm{C} & \mathrm{C} 5 \\ 17 & \mathrm{C} & \mathrm{C} 6 \\ 18 & \mathrm{H} & \mathrm{H} 2 \\ 19 & \mathrm{C} & \mathrm{C} 7 \\ 20 & \mathrm{H} & \mathrm{H} 8 \\ 21 & \mathrm{H} & \mathrm{H} 15 \\ 22 & \mathrm{H} & \mathrm{H} 16 \\ 23 & \mathrm{H} & \mathrm{H} 7 \\ 24 & \mathrm{H} & \mathrm{H} 3\end{array}$

-2.0138793
-1.3805876
0.0167825
1.0911077
0.4835563
1.8089138
1.6516626
1.0911077
0.4835563
1.6516626
1.8089138
-0.8394991
-1.4697626
-0.1127578
-1.4697626
-1.0961942
0.0758099
0.9849720
0.2646891
0.8251929
0.8251929
-0.6869611
-1.9797334
-2.0138793

$\odot .8607978$

$\odot .0000000$

0.0000000

1.5552528

2. 4556539

1. 5922443

1.6070082

$-1.5552528$

$-2.4556539$

$-1.6070082$

$-1.5922443$

๑. . 0000000

$-0.8765871$

0.0000000

$\odot .8765871$

$\odot .0000000$

$\odot .0000000$

$\odot .000000 \odot$

$\odot .0000000$

$\odot .8738305$

$-0.8738305$

0.0000000

$\odot .0000000$

$-\odot .8607978$
$-0.1687650$

๑. 0552191

$-1.2397626$

$-1.1159686$

$-1.1782563$

$-1.9329335$

$-0.1870706$

$-1.1159686$

$-1.1782563$

$-0.1870706$

$-1.9329335$

$-2.9313651$

$-3.0616775$

$-3.7402964$

$-3.0616775$

1. 5392687

2.1478516

1. 5692117

3. 6400316

3. 9631378

3. 9631378

4. 1602726

2. 1626351

$-0.1687650$

Point Group $=\mathrm{CS}$ Order $=1$ Nsymop $=2$

** Archive file written **

Memory Used: $10.673 \mathrm{Mb}$

Reason for exit: Successful completion

Ab-Initio Program CPU Time : 000:34:34.0

Ab-Initio Program Wall Time: 000:48:04.4

MacSPARTAN PRO Properties Program: (PowerPC)

Release 1.0.4

Reason for exit: Successful completion

Properties Program CPU Time : 000:00:01.0

Properties Program Wall Time: 000:00:01.9 


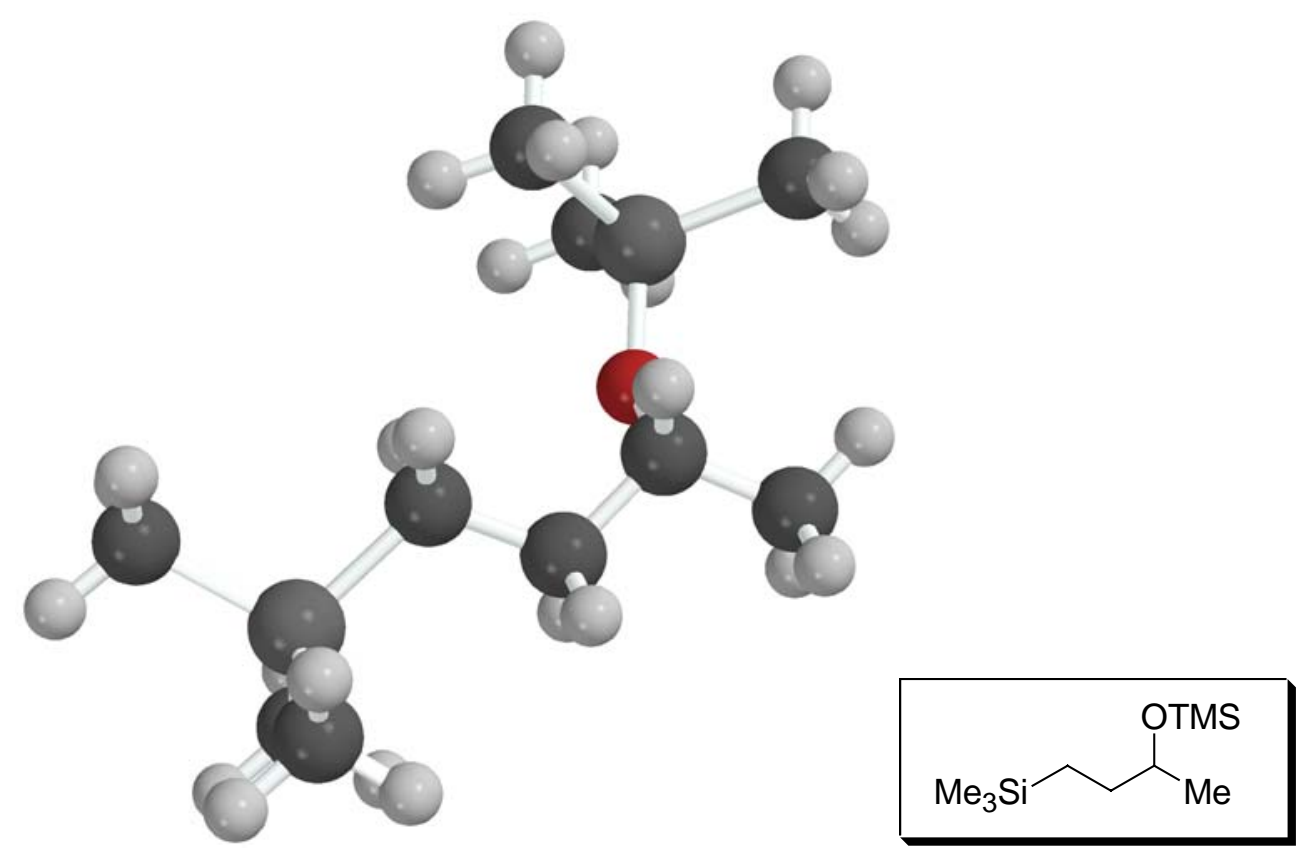

MacSPARTAN PRO Ab-Initio Program: (PowerPC)

Job run on machine : Woerpel G4(G4/466)

Release 1.0.4

Calculation started: Tue May 82007 2:23:22 PM

\section{TONYC1.1}

Run type: Geometry optimization

Model: RHF/6-31G*

Number of shells: 106

$65 \mathrm{~S}$ shells

28 SP shells

13 6D shells

Number of basis functions: 255

Number of electrons: 122

Number of heavy atoms: 13

Number of hydrogens: 26

Use of molecular symmetry disabled

Molecular charge: 0

Spin multiplicity: 1

Memory model: direct $22.4 \mathrm{Mb}$

Point Group = C1 Order $=1$ Nsymop $=1$

This system has 111 degrees of freedom

Hessian from 6-31G* calculation used.

Max. Max. Neg.

Cycle Energy Grad. Dist. Eigen

$\begin{array}{llll}1 & -1046.6257346 & 0.00177 & 0.02924\end{array}$

$\begin{array}{llll}2 & -1046.6259695 & 0.00237 & 0.02991\end{array}$

$\begin{array}{llll}3 & -1046.6262271 & 0.00247 & 0.03696\end{array}$

$\begin{array}{llll}4 & -1046.6264300 & 0.00338 & 0.04664\end{array}$

$\begin{array}{llll}5 & -1046.6265929 & 0.00496 & 0.02146\end{array}$

$\begin{array}{llll}6 & -1046.6267321 & 0.00623 & 0.03159\end{array}$

$\begin{array}{llll}7 & -1046.6269857 & 0.00472 & 0.02864\end{array}$ 


\begin{tabular}{|c|c|c|}
\hline $8-1046.6271390$ & & \\
\hline 9 -1046.6274121 & 0.00316 & \\
\hline o -1046.6277407 & & \\
\hline 1 -1046.6280010 & 00475 & \\
\hline 2 -1046.6282694 & & \\
\hline 3 -1046.6284915 & 502 & \\
\hline 7540 & & \\
\hline 5 -1046.6289604 & & \\
\hline 6 -1046.6291363 & & \\
\hline 7 -1046.6292668 & & \\
\hline $8-104$ & & \\
\hline $9-104$ & & \\
\hline-1046.6296 & 0.0 & \\
\hline-104 & & \\
\hline 6.6297871 & & \\
\hline $3-104$ & & \\
\hline $4-10$ & & \\
\hline $5-104$ & & \\
\hline $6-104$ & & \\
\hline $7-104$ & & \\
\hline $28-104$ & & \\
\hline-104 & & \\
\hline $30-10$ & & \\
\hline $1-10$ & & \\
\hline $2-10$ & & \\
\hline $3-10$ & & \\
\hline $4-104$ & & \\
\hline $35-104$ & & \\
\hline-104 & & \\
\hline $37-104$ & & \\
\hline $38-104$ & & \\
\hline $9-10$ & & \\
\hline $0-104$ & & \\
\hline $1-104$ & & \\
\hline $42-104$ & & \\
\hline-104 & & \\
\hline $44-1046.6304$ & & \\
\hline $45-1046.6304$ & & \\
\hline $46-104$ & & \\
\hline $47-104$ & 33 & \\
\hline-104 & & \\
\hline $49-1046.63$ & 0.00029 & \\
\hline $50-1046.63$ & & \\
\hline $1-1046.6$ & & \\
\hline-1046.6304655 & & \\
\hline-1046.6304697 & 0.00 & \\
\hline-1046.6304761 & 0.00 & \\
\hline-1046.6304811 & & \\
\hline-1046.6304835 & 0.00037 & 0.00230 \\
\hline $57-1046.6304862$ & & \\
\hline $58-1046.6304880$ & 0.00033 & 0.00138 \\
\hline-1046.6304891 & & \\
\hline-1046.6304899 & 0.00024 & \\
\hline & & \\
\hline
\end{tabular}

TONYC1.1 
$\mathrm{E}(\mathrm{HF})=-1046.6304909$ a.u.

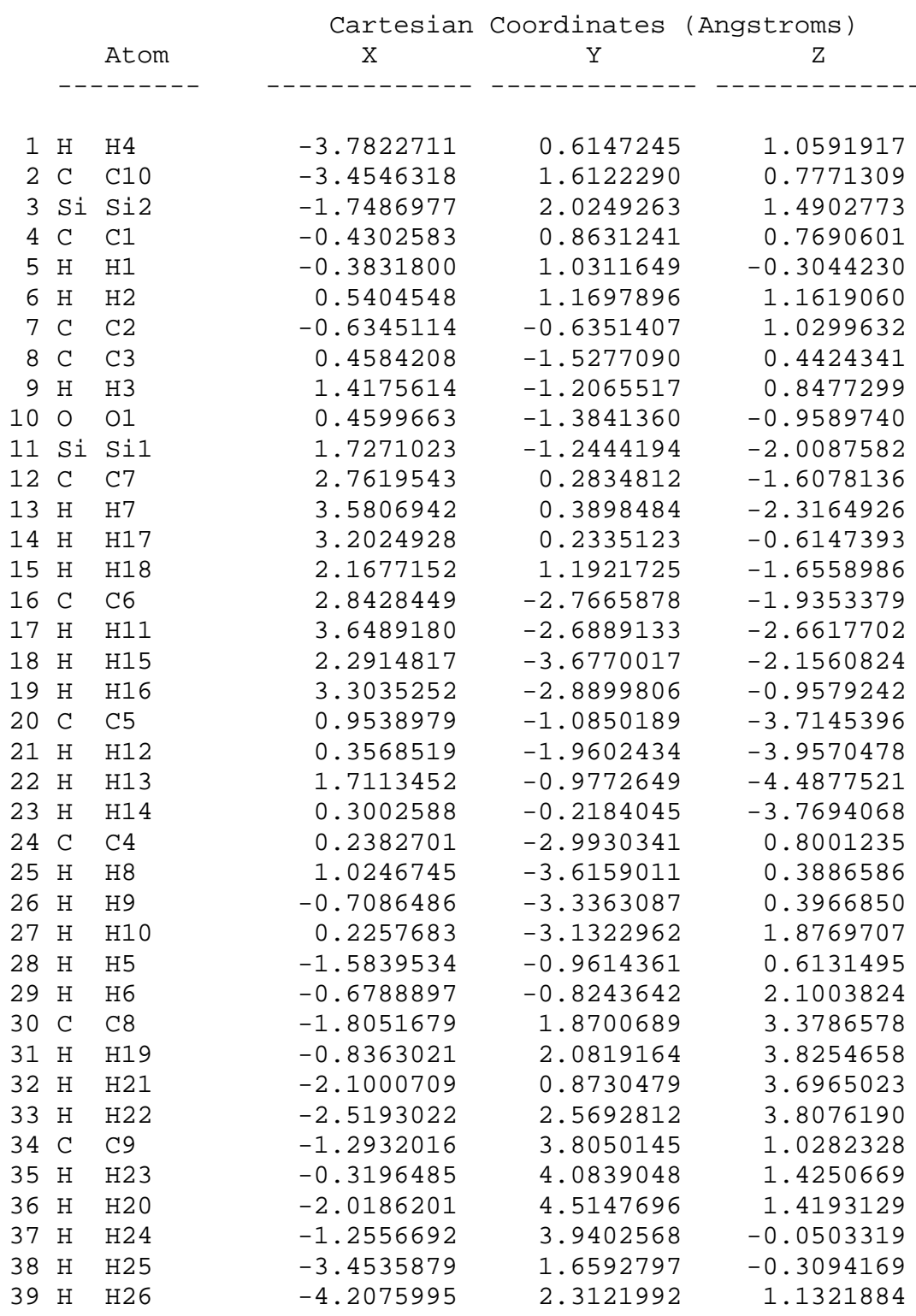

Point Group $=\mathrm{C} 1$ Order $=1$ Nsymop $=1$

** Archive file written **

Memory Used: $22.746 \mathrm{Mb}$

Reason for exit: Successful completion

Ab-Initio Program CPU Time : 021:58:43.8

Ab-Initio Program Wall Time: 031:39:41.0

MacSPARTAN PRO Properties Program: (PowerPC)

Release 1.0.4 
Reason for exit: Successful completion

Properties Program CPU Time : 000:00:03.2

Properties Program Wall Time: 000:00:04.8

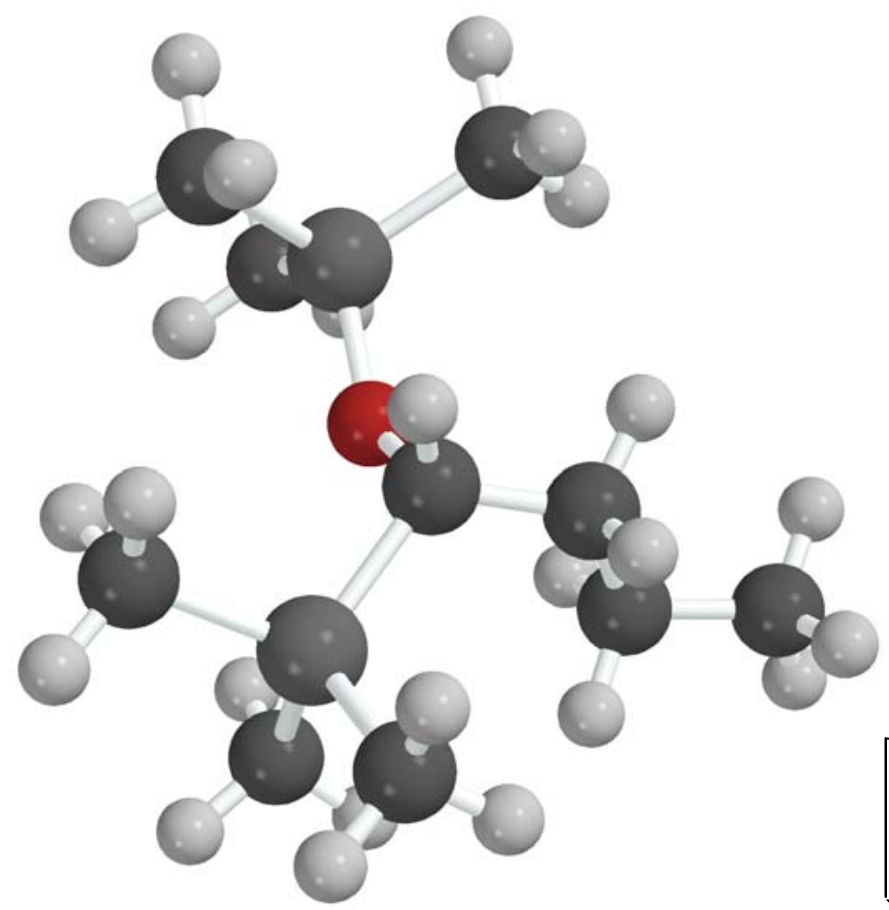

MacSPARTAN PRO Ab-Initio Program: (PowerPC)

Job run on machine : Woerpel G4(G4/466)

Calculation started: Wed May 92007 9:58:27 PM

\section{TONYC2.1}

Run type: Geometry optimization

Model: RHF/6-31G*

Number of shells: 106

$65 \mathrm{~S}$ shells

28 SP shells

13 6D shells

Number of basis functions: 255

Number of electrons: 122

Number of heavy atoms: 13

Number of hydrogens: $\quad 26$

Use of molecular symmetry disabled

Molecular charge: 0

Spin multiplicity: 1

Memory model: direct $22.4 \mathrm{Mb}$

Point Group = C1 Order $=1$ Nsymop $=1$

This system has 111 degrees of freedom

Hessian from AM1 calculation used.

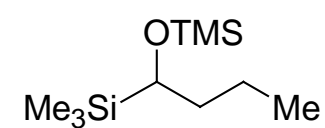

Release 1.0.4 


\begin{tabular}{|c|c|c|c|}
\hline \multirow[b]{2}{*}{ Cycle } & Max. & \multicolumn{2}{|r|}{ Neg. } \\
\hline & Energy & Grad. & Dist. Eigen \\
\hline 1 & -1046.5675551 & 0.05883 & 0.01646 \\
\hline 2 & -1046.5965216 & 0.03255 & 0.01775 \\
\hline 3 & -1046.6059136 & 0.00963 & 0.01877 \\
\hline 4 & -1046.6090599 & 0.00606 & 0.02197 \\
\hline 5 & -1046.6107576 & 0.00459 & 0.02215 \\
\hline 6 & -1046.6118926 & 0.00384 & 0.02081 \\
\hline 7 & -1046.6127515 & 0.00349 & 0.02587 \\
\hline 8 & -1046.6134709 & 0.00254 & 0.01773 \\
\hline 9 & -1046.6139391 & 0.00445 & 0.03229 \\
\hline 10 & -1046.6144614 & 0.00257 & 0.01881 \\
\hline 11 & -1046.6148906 & 0.00266 & 0.02849 \\
\hline 12 & -1046.6152712 & 0.00349 & 0.01886 \\
\hline 13 & -1046.6156219 & 0.00345 & 0.01498 \\
\hline 14 & -1046.6158728 & 0.00342 & 0.01824 \\
\hline 15 & -1046.6161157 & 0.00407 & 0.02551 \\
\hline 16 & -1046.6162802 & 0.00338 & 0.02000 \\
\hline 17 & -1046.6164489 & 0.00230 & 0.02424 \\
\hline 18 & -1046.6166094 & 0.00191 & 0.02145 \\
\hline 19 & -1046.6167371 & 0.00297 & 0.02520 \\
\hline 20 & -1046.6168725 & 0.00218 & 0.03994 \\
\hline 21 & -1046.6168584 & 0.00433 & 0.01346 \\
\hline 22 & -1046.6170406 & 0.00351 & 0.05208 \\
\hline 23 & -1046.6169214 & 0.00500 & 0.01624 \\
\hline 24 & -1046.6171975 & 0.00185 & 0.01571 \\
\hline 25 & -1046.6172555 & 0.00265 & 0.00618 \\
\hline 26 & -1046.6173453 & 0.00105 & 0.00976 \\
\hline 27 & -1046.6174043 & 0.00160 & 0.02365 \\
\hline 28 & -1046.6174952 & 0.00262 & 0.03062 \\
\hline 29 & -1046.6175491 & 0.00311 & 0.01637 \\
\hline 30 & -1046.6176127 & 0.00241 & 0.03251 \\
\hline 31 & -1046.6176679 & 0.00218 & 0.02518 \\
\hline 32 & -1046.6177504 & 0.00343 & 0.01349 \\
\hline 33 & -1046.6178387 & 0.00136 & 0.02083 \\
\hline 34 & -1046.6179088 & 0.00140 & 0.02446 \\
\hline 35 & -1046.6179833 & 0.00123 & 0.03252 \\
\hline 36 & -1046.6180183 & 0.00211 & 0.01035 \\
\hline 37 & -1046.6180263 & 0.00214 & 0.00369 \\
\hline 38 & -1046.6180779 & 0.00101 & 0.00926 \\
\hline 39 & -1046.6180916 & 0.00125 & 0.00358 \\
\hline 40 & -1046.6181092 & 0.00092 & 0.00610 \\
\hline 41 & -1046.6181240 & 0.00071 & 0.00447 \\
\hline 42 & -1046.6181347 & 0.00042 & 0.00363 \\
\hline 43 & -1046.6181436 & 0.00074 & 0.00151 \\
\hline 44 & -1046.6181478 & 0.00118 & 0.00062 \\
\hline 45 & -1046.6181543 & 0.00038 & 0.00040 \\
\hline 46 & -1046.6181576 & 0.00034 & 0.00061 \\
\hline 47 & -1046.6181616 & 0.00028 & 0.00108 \\
\hline 48 & -1046.6181666 & 0.00026 & 0.00208 \\
\hline 49 & -1046.6181721 & 0.00028 & 0.00418 \\
\hline
\end{tabular}

TONYC2.1

$\mathrm{E}(\mathrm{HF})=-1046.6181721$ a.u. 


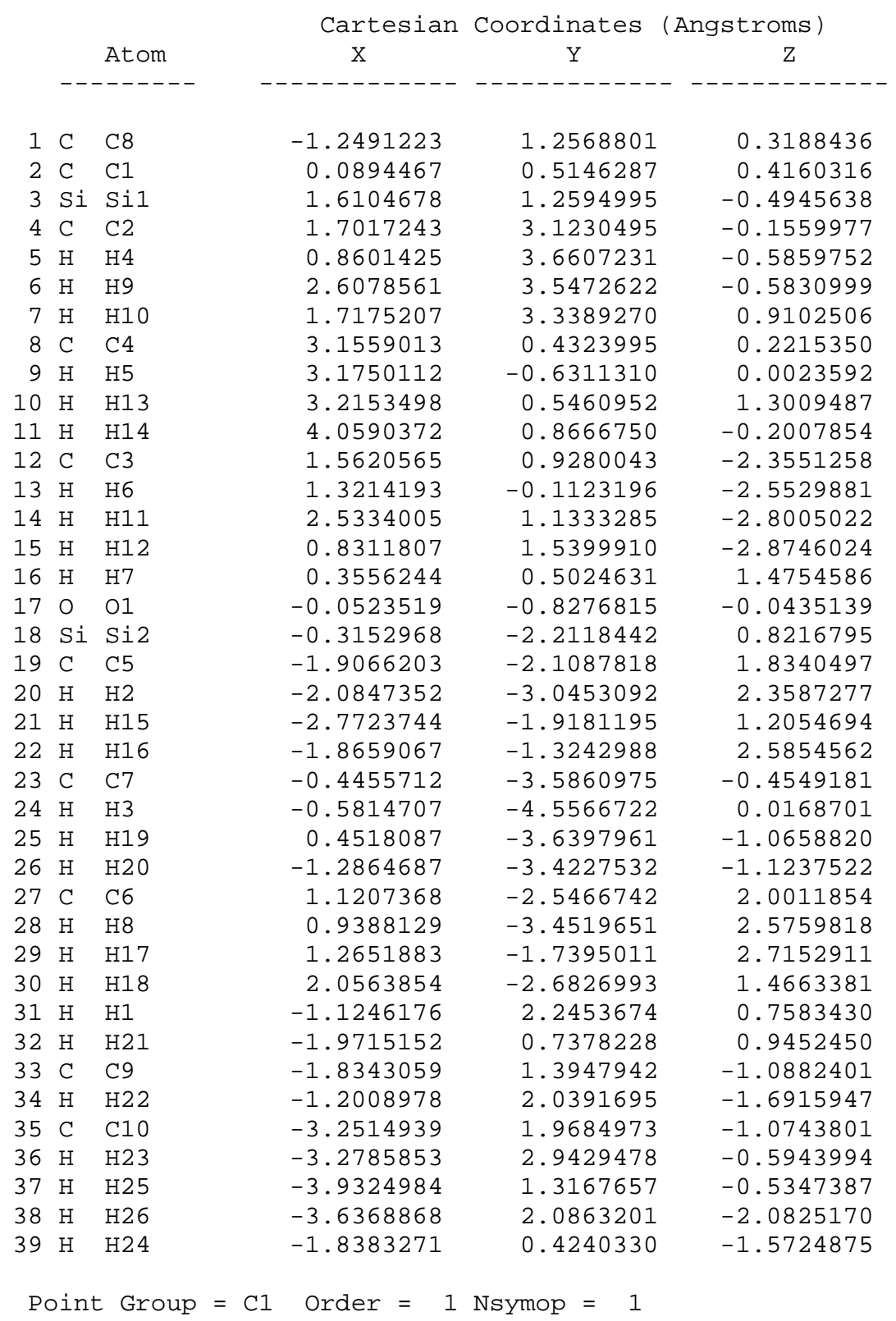

** Archive file written **

Memory Used: $22.746 \mathrm{Mb}$

Reason for exit: Successful completion

Ab-Initio Program CPU Time : 020:28:36.4

Ab-Initio Program Wall Time: 029:20:21.4

MacSPARTAN PRO Properties Program: (PowerPC)

Reason for exit: Successful completion

Properties Program CPU Time : 000:00:03.2

Properties Program Wall Time: 000:00:04.7

Release 1.0.4 